\title{
TITLE:
}

\section{Three-dimensional exact coherent states in rotating channel flow}

AUTHOR(S):

Wall, D. P; Nagata, M.

\section{CITATION:}

Wall, D. P ... [et al]. Three-dimensional exact coherent states in rotating channel flow. Journal of Fluid Mechanics 2013, 727: 533-581

ISSUE DATE:

2013-07

URL:

http://hdl.handle.net/2433/193964

RIGHT:

C2013 Cambridge University Press 


\title{
Three-dimensional exact coherent states in rotating channel flow
}

\author{
D. P. Wall $\dagger$ and M. Nagata \\ Department of Aeronautics and Astronautics, Graduate School of Engineering, University of Kyoto, \\ Kyoto 606-8501, Japan \\ (Received 16 August 2012; revised 8 March 2013; accepted 8 May 2013; \\ first published online 28 June 2013)
}

Three-dimensional exact, finite-amplitude solutions are presented for the problem of channel flow subject to a system rotation about a spanwise axis. The solutions are of travelling wave form, and may bifurcate as tertiary flows from the twodimensional streamwise-independent secondary flow, or as secondary flows directly from the basic flow. For the tertiary flows, we consider solutions of spanwise superharmonic and subharmonic type. We distinguish flows on the basis of symmetry, originating eigenmode and major solution branch, and thus identify 15 distinct flows: 5 superharmonic tertiary, 5 subharmonic tertiary and 5 secondary flows. The tertiary flows all feature a single layer of vortical structures in the spanwise-wall-normal plane, the secondary flows feature single-, double-, triple- or quadruple-layer flow structures in this plane. All flows feature low-speed streamwise-orientated streaks in the streamwise velocity component and/or pulses of low-speed streamwise velocity. The streaks may be sinusoidal or varicose. Sinusoidal streaks are flanked by staggered streamwise vortices, varicose streaks and pulses are flanked by aligned vortices. A comparison with previous simulation and experimental studies finds that the simplest three-dimensional flows observed previously correspond to superharmonic tertiary flows bifurcating from the upper branch of the secondary flow. The mean absolute vorticity of the present flows is also considered. A flattening of the profile of this vorticity is observed in the central region of the channel for two-dimensional secondary and many of the three-dimensional flows, with two-step profiles also observed. This phenomenon is attributed to mixing of the vorticity across zones of the channel in which streamwise vortex structures exist, and is demonstrated by a two-dimensional model. The phenomenon appears to be distinct to that observed in fully turbulent rotating channel flows.

Key words: absolute vorticity, finite-amplitude solutions, transition to turbulence

\section{Introduction}

Understanding the effects of rotation on fluid flows is important to many fields of study related to fluid mechanics, including geophysical flows, oceanography and meteorology (see, for example, Hopfinger \& Linden 1990; Lesieur, Yaglom \& David 2002) as well as being important to many engineering applications, including rotating 
machinery such as compressive impellers, hydraulic turbines or radial pump flows, or instruments that measure mass flow rates based on the Coriolis effect.

The present study considers the problem of the flow of fluid through a parallelsided channel subject to a system rotation about a spanwise axis. This problem has previously been studied from a linear stability viewpoint (Lezius \& Johnstone 1976; Alfredsson \& Persson 1989), and flow regimes have been clarified by numerical simulation studies (see, for example, Finlay 1990, 1992; Yang \& Kim 1991; Kristoffersen \& Andersson 1993; Yanase \& Kaga 2004) as well as through experimental studies (see, for example, Alfredsson \& Persson 1989; Matsubara \& Alfredsson 1998). These studies suggest that the basic flow first loses stability to a two-dimensional streamwise-invariant steady secondary flow consisting of streamwiseorientated roll cells. Using a bifurcation approach, Wall \& Nagata (2006) reported on exact, finite-amplitude solutions for these two-dimensional roll cells. The flow bifurcating from the linear critical point was found to be stable in agreement with the results of direct numerical simulation (DNS) and experimental studies, while more generally a closed Eckhaus boundary (here an Eckhaus disturbance means a streamwise-independent secondary disturbance) was identified within which the twodimensional secondary flows were stable, while outside the boundary these flows were found to be unstable. In addition, a rich variety of bifurcation connections was demonstrated to exist between the two-dimensional solutions.

With increasing Reynolds number (or rotation number), as found experimentally by Alfredsson \& Persson (1989), and through the simulations of Finlay (1990) and Yang \& Kim (1991) for example, a secondary instability is manifested as a wavy travelling wave disturbance imposed on the streamwise roll cells. Two such three-dimensional tertiary travelling wave flows are identified by these studies: one is distinguished by a streamwise wavenumber of the same order as the spanwise wavenumber; while the other is distinguished by a streamwise wavenumber of an order of magnitude smaller than the spanwise wavenumber. Finlay (1990) proposed that these flows are associated with the loss of stability of the secondary flow to two distinct eigenmodes. For certain values of the Reynolds number and rotation number, flow regimes that include both types of the above three-dimensional flows are also found to exist, while Finlay (1990) also describes a flow that does not appear to correspond to either of these two flow types. More recently, Yanase \& Kaga (2004) have found that the two-dimensional steady solution may also lose stability to a periodic solution in which the flow structure oscillates between two and four streamwise-independent streamwiseorientated vortical structures. The reader is also referred to Wall \& Nagata (2006) for further details of the background to this problem.

There are clearly similarities between the transition of the present flow and transitions that occur in Taylor-Couette flow (see the review of DiPrima \& Swinney (1985) for example). For the latter configuration instability of the basic flow is also first observed in the appearance of steady streamwise-independent streamwise rolls, with streamwise-dependent tertiary flows then appearing due to secondary instabilities of the secondary flows. At higher Reynolds numbers the flow becomes turbulent, but regimes in which turbulence is embedded in streamwise roll structures can be observed for this flow as well as for the present flow. Perhaps due to the relative ease of conducting the corresponding experiments, closed flows such as Taylor-Couette flow have hitherto received considerable research attention, but more recently open flows such as the present flow, which are no less important in terms of their applications, have also been receiving attention. Tsukahara, Tillmark \& Alfredsson (2010) have, for example, recently conducted experiments on the related problem of plane Couette flow 
subject to a spanwise system rotation. They identified 17 distinct flow states, including laminar and turbulent flows, as well as flows featuring both of these regimes, and were able to confirm the existence of the three-dimensional wavy vortex flow predicted theoretically by Nagata (1998). It is also noted that a similar transitional sequence (basic flow $\Rightarrow$ two-dimensional steady streamwise roll cells $\Rightarrow$ three-dimensional wavy vortex flows) along with embedded turbulent roll cells can also be observed in curved channel flow, see, for example, the study of Finlay, Keller \& Ferziger (1988).

The present study examines the problem of Poiseuille channel flow subject to a spanwise system rotation using a bifurcation approach to find exact, finite-amplitude tertiary flow solutions to the governing equations (the Navier-Stokes equations expressed in a rotating frame of reference). Over the past two decades a number of such solutions have been presented for wall-bounded shear flows for which the basic state is linearly stable (these include: plane Couette flow Nagata (1990) and Gibson, Halcrow \& Cvitanovic (2009); pipe flow Wedin \& Kerswell (2004) and Faisst \& Eckhardt (2003)) or for which transition bypasses the linear stability mechanism in noisy practical applications (see the study of Waleffe (2001) for plane Poiseuille flow, for example). In such flows transition from the basic state laminar flow occurs abruptly, triggered by finite-amplitude disturbances. Consistent with these simulation and experimental findings, the exact finite-amplitude solutions do not bifurcate directly from the basic state, but instead arise in saddle-node bifurcations, and tend to first appear at Reynolds numbers below typical transition values. Investigations are continuing into the precise role such solutions play, and in particular whether transitional flows can be described in terms of trajectories that travel around the exact coherent states in phase space (see Kerswell (2005) for a recent review of progress in pipe flow).

However, in contrast to such abrupt transition processes, for the present configuration, as for the other flows described above with similar transition processes, the flow bifurcates from the basic flow in sequence through progressively more complicated flows as the value of a physical parameter is changed. The previous theoretical studies of the present problem described above have described the loss of stability of the basic flow, the structure of the two-dimensional secondary flows and their connections. The present study extends these results by exploring the threedimensional tertiary flows that arise upon the loss of stability of the previously studied secondary flows. All of the presented tertiary flows bifurcate from the secondary flow with spanwise wavenumber $\grave{\beta}=2.5$, which was studied extensively in Wall \& Nagata (2006). This case is sufficient to represent all the tertiary flows bifurcating from secondary flows with $\grave{\beta}$ in the range $2 \leqslant \grave{\beta} \leqslant 4$ that can be found by the present technique, which includes in particular the interval $(2.35 \leqslant \grave{\beta} \leqslant 3.45)$ of stable secondary flows. For completeness, three-dimensional travelling wave secondary flows that bifurcate directly from the basic state flow are also considered, and a qualitative and quantitative comparison of the present solutions with previous experimental and DNS results is also presented. Furthermore, we investigate whether a phenomenon observed for turbulent rotating channel flow, in which mean absolute vorticity approaches zero across a central region of the channel, can also be observed for the present transitional flows.

\section{Mathematical formulation}

The physical configuration of the channel is shown in figure 1. As shown in the figure, we adopt a Cartesian coordinate system whose origin is located on the 


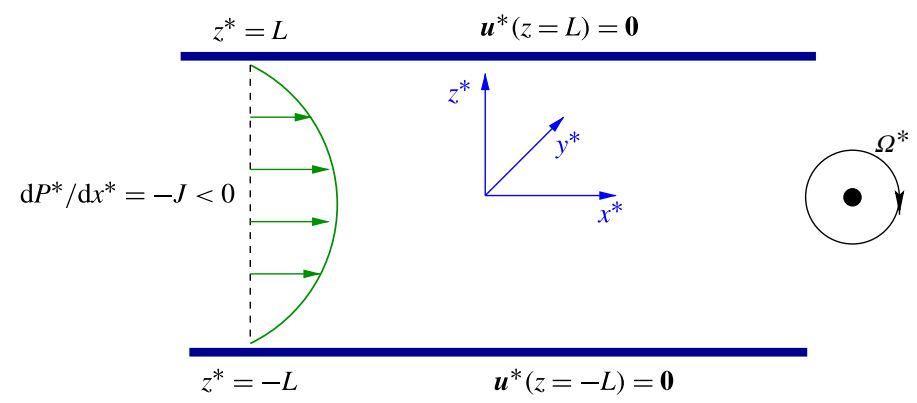

FIGURE 1. (Colour online) Configuration of the channel in dimensional coordinates.

centreline of the channel, with the ordinates $x_{1}^{*}=x^{*}, x_{2}^{*}=y^{*}$ and $x_{3}^{*}=z^{*}$ representing the distances in the streamwise, spanwise and wall-normal directions, respectively, in a frame rotating with the channel at a constant rate, $\Omega^{*}$, about a spanwise axis, where a $\operatorname{star}(*)$ denotes a dimensional variable. We consider the flow of an incompressible fluid between the channel walls located at $z^{*}= \pm L$, where $L$ denotes half the channel width, and so the problem is governed by the Navier-Stokes equations expressed in a rotating frame, together with the incompressibility condition,

$$
\begin{aligned}
\frac{\partial u_{i}^{*}}{\partial t^{*}}+u_{j}^{*} \frac{\partial u_{i}^{*}}{\partial x_{j}^{*}} & =-\frac{1}{\rho} \frac{\partial}{\partial x_{i}^{*}}\left(p^{*}-\frac{\rho}{2}\left|\Omega^{*} \times \boldsymbol{x}^{*}\right|^{2}\right)+v \nabla^{* 2} u_{i}^{*}+2 \epsilon_{i, j, k} u_{j}^{*} \Omega_{k}^{*}, \\
\frac{\partial u_{i}^{*}}{\partial x_{i}^{*}} & =0
\end{aligned}
$$

where the variables $u_{i}, p$ and $t$ denote the component of velocity in the $x_{i}$ direction (we shall also use $\left.\boldsymbol{u}=(u, v, w)=\left(u_{1}, u_{2}, u_{3}\right)\right)$, pressure and time, respectively, and $\rho$ and $v$ denote the constant density and kinematic viscosity. It is convenient to introduce the modified pressure

$$
\pi^{*}=p^{*}-\frac{\rho}{2}\left|\Omega^{*} \times \boldsymbol{x}^{*}\right|^{2}
$$

and we consider a flow driven by a constant imposed mean (modified) pressure gradient, $-J(J>0)$ in the positive streamwise direction, and subject to no-slip at the channel walls,

$$
u_{i}^{*}\left(z^{*}= \pm L\right)=0
$$

The following non-dimensional variables are adopted: $\boldsymbol{x}=\boldsymbol{x}^{*} / L, \boldsymbol{u}=\boldsymbol{u}^{*} / V, \quad p=$ $\pi^{*} / \rho V^{2}, t=t^{*} V / L$, where we select the velocity scale $V=v / L$. We shall also make use of the unit vectors $\boldsymbol{i}, \boldsymbol{j}$ and $\boldsymbol{k}$ in the $x, y$ and $z$ directions, respectively. We are left to solve the dimensionless equations

$$
\begin{gathered}
\frac{\partial u_{i}}{\partial t}+u_{j} \frac{\partial u_{i}}{\partial x_{j}}+\frac{\partial p}{\partial x_{i}}-\frac{\partial^{2} u_{i}}{\partial x_{j} \partial x_{j}}+\Omega\left(u_{3} \delta_{i, 1}-u_{1} \delta_{i, 3}\right)=0, \\
\frac{\partial u_{j}}{\partial x_{j}}=0
\end{gathered}
$$


Three-dimensional exact coherent states in rotating channel flow

where $\Omega=2 \Omega^{*} L^{2} / \nu$ is a rotation number, subject to the no-slip boundary conditions at the channel walls,

$$
\boldsymbol{u}(z= \pm 1)=\mathbf{0} .
$$

Seeking a solution in the form $\boldsymbol{u}=\left(u_{0}(z), 0,0\right), p=p_{0}(x, z)$, we obtain the basic state $u_{0}(z)=R\left(1-z^{2}\right), \partial p_{0} / \partial x=-2 R, \partial p_{0} / \partial z=\Omega R\left(1-z^{2}\right)$, where $R=L^{3} J / 2 \rho v^{2}$ denotes the Reynolds number. Note that, in contrast to the non-rotating case, the basic state includes a non-zero pressure gradient in the wall-normal direction.

The present study seeks to find solutions other than the basic state, for which, in the present bifurcation approach, it is convenient to introduce and solve for finiteamplitude disturbances. Accordingly, we seek a solution in the form $\boldsymbol{u}=\boldsymbol{u}_{\mathbf{0}}+\hat{\boldsymbol{u}}, p=$ $p_{0}+\hat{p}$, and it is convenient to separate the disturbance into mean and fluctuating parts, $\hat{\boldsymbol{u}}=(\breve{U}, \breve{V}, \breve{W})+(\check{u}, \check{v}, \check{w}), \hat{p}=\check{P}+\check{p}$, where the mean variables $\check{\boldsymbol{U}}=(\check{U}, \breve{V}, \breve{W})$ and $\breve{P}$ are obtained by spatially averaging $\hat{\boldsymbol{u}}=(\hat{u}, \hat{v}, \hat{w})$ and $\hat{p}$, respectively, in both the $x$ and $y$ directions. Substituting into (2.5) and (2.6) for $u, v, w$ and $p$, it is straightforward to derive $\breve{W} \equiv 0$, while we can exploit the incompressibility condition to express the fluctuating velocity as $\check{u}=\nabla \times(\nabla \times(\phi \boldsymbol{k}))+\nabla \times(\psi \boldsymbol{k})$. Upon applying operators ' $\boldsymbol{k} \cdot \boldsymbol{\nabla} \times(\boldsymbol{\nabla} \times$ ' and ' $\boldsymbol{k} \cdot \nabla \times$ ' to (2.5), we then obtain

$$
\begin{aligned}
& \frac{\partial\left(\nabla^{2} \Delta_{2} \phi\right)}{\partial t}+\left(\left[U \nabla^{2}-\frac{\partial^{2} U}{\partial z^{2}}\right] \partial_{x}+\left[\check{V} \nabla^{2}-\frac{\partial^{2} \check{V}}{\partial z^{2}}\right] \partial_{y}-\nabla^{4}\right) \Delta_{2} \phi \\
& +\boldsymbol{k} \cdot \nabla \times(\nabla \times \check{\boldsymbol{u}} \cdot \nabla \check{\boldsymbol{u}})+\Omega \Delta_{2} \psi_{y}=0, \\
& \frac{\partial\left(\Delta_{2} \psi\right)}{\partial t}+\left(U \partial_{x}+\check{V} \partial_{y}\right) \Delta_{2} \psi+\left(\frac{\partial \check{V}}{\partial z} \partial_{x}-\left[\frac{\partial U}{\partial z}+\Omega\right] \partial_{y}-\nabla^{2}\right) \Delta_{2} \phi \\
& -\boldsymbol{k} \cdot \nabla \times \check{\boldsymbol{u}} \cdot \nabla \check{\boldsymbol{u}}=0,
\end{aligned}
$$

where $\Delta_{2}$ is the two-dimensional Laplacian, $\Delta_{2}=\partial^{2} / \partial x^{2}+\partial^{2} / \partial y^{2}$, and $U=$ $u_{0}(z)+\check{U}(z, t)$ is the mean flow. Upon taking the spanwise-streamwise spatial average of (2.5), we also derive

$$
\begin{aligned}
\frac{\partial \check{U}}{\partial t}-\frac{\partial}{\partial z} \overline{\Delta_{2} \phi\left(\phi_{x z}+\psi_{y}\right)} & =\frac{\partial^{2} \check{U}}{\partial z^{2}}, \\
\frac{\partial \check{V}}{\partial t}-\frac{\partial}{\partial z} \overline{\Delta_{2} \phi\left(\phi_{y z}-\psi_{x}\right)} & =\frac{\partial^{2} \check{V}}{\partial z^{2}}, \\
\Omega \check{U}-\frac{\partial}{\partial z} \overline{\left(\Delta_{2} \phi\right)^{2}} & =\frac{\partial \check{P}}{\partial z},
\end{aligned}
$$

where here $\bar{f}=\left(\alpha \beta / 4 \pi^{2}\right) \int_{0}^{2 \pi / \alpha} \int_{0}^{2 \pi / \beta} f \mathrm{~d} x \mathrm{~d} y$, in which $\alpha$ and $\beta$, respectively, denote the streamwise and spanwise wavenumbers defined in $\S 4$. Since $\check{P}$ only appears in (2.12), this equation can be treated as an expression for $\partial \breve{P} / \partial z$ in terms of $\phi, \psi, \breve{U}$ and $\breve{V}$, with these latter four variables obtained by solving (2.8)-(2.11). These equations are solved subject to the no-slip boundary conditions at the channel walls,

$$
\begin{gathered}
\psi( \pm 1)=\phi( \pm 1)=\left.\frac{\partial \phi}{\partial z}\right|_{z=-1}=\left.\frac{\partial \phi}{\partial z}\right|_{z=1}=0, \\
\check{U}(z= \pm 1)=\check{V}(z= \pm 1)=0 .
\end{gathered}
$$


In practice, for all of the three-dimensional solutions found in the present study, as for the two-dimensional secondary flows described by Wall \& Nagata (2006), we have that the symmetries of these solutions are such that $\overline{\Delta_{2} \phi\left(\phi_{y z}-\psi_{x}\right)} \equiv 0$, and so no spanwise mean flow distortion is possible, $\check{V} \equiv 0$. (The symmetries of the present solutions are detailed in appendix A.)

\section{Two-dimensional steady solutions and their stability}

Both experimental studies (see, for example, Alfredsson \& Persson 1989) and DNS studies (see for example Yang \& Kim 1991) find that the basic flow first loses stability to a steady, two-dimensional flow featuring streamwise-independent roll-cell structures. Such flows were extensively investigated by Wall \& Nagata (2006). The present study instead concerns three-dimensional travelling wave flows; both the tertiary flows that arise upon the loss of stability of these two-dimensional secondary flows, as well as the secondary flows that bifurcate directly from the basic flow. In order to understand the origins of the former, it is necessary to briefly review the formulation of the two-dimensional secondary flows and their stability. Such steady secondary flows may be expressed in the form $\boldsymbol{u}=\boldsymbol{u}_{2 D}(\boldsymbol{y}, \boldsymbol{z})=\boldsymbol{u}_{\mathbf{0}}+\check{U} \boldsymbol{i}+\nabla \times(\nabla \times(\phi \boldsymbol{k}))+\nabla \times(\psi \boldsymbol{k})$ where

$$
\begin{aligned}
\phi(y, z) & =\sum_{l=0}^{\infty} \sum_{n=-\infty}^{\infty} \Phi_{l, n} \mathrm{e}^{\mathrm{i} n \grave{\beta} y} T_{l}(z)\left(1-z^{2}\right)^{2}, \\
\psi(y, z) & =\sum_{l=0}^{\infty} \sum_{n=-\infty}^{\infty} \Psi_{l, n} \mathrm{e}^{\mathrm{i} n \grave{\beta} y} T_{l}(z)\left(1-z^{2}\right), \\
\check{U}(z) & =\sum_{k=0}^{\infty} \gamma_{k} T_{k}(z)\left(1-z^{2}\right),
\end{aligned}
$$

while their stability may be considered by adding a disturbance of the form

$$
\begin{aligned}
& \tilde{\phi}=\sum_{l=0}^{\infty} \sum_{n=-\infty}^{\infty} \tilde{a}_{l, n} \mathrm{e}^{\mathrm{i} n \hat{\beta} y+\mathrm{i} d x+\mathrm{i} b y+\sigma t} T_{l}(z)\left(1-z^{2}\right)^{2}, \\
& \tilde{\psi}=\sum_{l=0}^{\infty} \sum_{n=-\infty}^{\infty} \tilde{b}_{l, n} \mathrm{e}^{\mathrm{i} i \grave{\beta} y+\mathrm{i} d x+\mathrm{i} b y+\sigma t} T_{l}(z)\left(1-z^{2}\right),
\end{aligned}
$$

in which $d$ and $b$ thus denote the streamwise and spanwise wavenmbers of the secondary disturbance, respectively. Upon requiring the overall flow including this disturbance to satisfy the governing equations (2.8) and (2.9), in which products of the disturbances are neglected, subject to boundary conditions (2.4), we derive an eigenvalue problem for $\sigma=f(d, b ; R, \Omega, \grave{\beta})$. As discussed by Wall \& Nagata (2006), this eigenvalue problem is periodic in $b$ with period $\grave{\beta}$. In the present study, we restrict attention to spanwise superharmonic (i.e. $b=0)$ and spanwise subharmonic $(b=\grave{\beta} / 2)$ secondary disturbances. We shall focus in particular on tertiary flows arising in bifurcations from the two-dimensional solution branch with parameters $\Omega=22.1325$ and $\grave{\beta}=2.5$, which was studied in detail by Wall \& Nagata (2006) and, as is discussed in $\S 5$, is sufficient to describe all of the tertiary flows bifurcating from secondary flows with $2 \leqslant \grave{\beta} \leqslant 4$ that the present approach is able to obtain. With the exception of the three-dimensional secondary flows presented in $\S 5.3$, which bifurcate directly from 
Three-dimensional exact coherent states in rotating channel flow

the basic flow, and the flows prepared for specific comparison with previous authors' work in $\$ 7$, all of the three-dimensional flows presented in this study bifurcate directly from this two-dimensional secondary flow.

\section{Three-dimensional travelling wave solutions}

We seek solutions to (2.8)-(2.10) subject to boundary conditions (2.13) and (2.14) in the Fourier-Chebyshev travelling wave form

$$
\begin{aligned}
\phi(x, y, z, t) & =\sum_{l=0}^{\infty} \sum_{m=-\infty}^{\infty} \sum_{n=-\infty}^{\infty} a_{l, m, n} \mathrm{e}^{\mathrm{i}(m \alpha(x-c t)+n \beta y)} T_{l}(z)\left(1-z^{2}\right)^{2}, \\
\psi(x, y, z, t) & =\sum_{l=0}^{\infty} \sum_{m=-\infty}^{\infty} \sum_{n=-\infty}^{\infty} b_{l, m, n} \mathrm{e}^{\mathrm{i}(m \alpha(x-c t)+n \beta y)} T_{l}(z)\left(1-z^{2}\right), \\
\check{U}(z) & =\sum_{k=0}^{\infty} C_{k} T_{k}(z)\left(1-z^{2}\right),
\end{aligned}
$$

where $\alpha$ and $\beta$ denote streamwise and spanwise wavenumbers, respectively, $c$ denotes the wavespeed, while $T_{k}(z)=\cos (k \arccos (z))$ is the $k$ th Chebyshev polynomial of the first kind. The amplitude coefficients $a_{l, m, n}$ and $b_{l, m, n}$ are in general complex, but in requiring a real solution we have that $\phi^{\star}=\phi$ and $\psi^{\star}=\psi$, where $Z^{\star}$ denotes the complex conjugate of $Z \in \mathbb{C}$, so we have

$$
\begin{aligned}
& \sum_{l=0}^{\infty} \sum_{m=\infty}^{-\infty} \sum_{n=\infty}^{-\infty} a_{l,-m,-n}^{\star} \mathrm{e}^{\mathrm{i}(m \alpha(x-c t)+n \beta y)} T_{l}(z)\left(1-z^{2}\right)^{2} \\
& \quad=\sum_{l=0}^{\infty} \sum_{m=-\infty}^{\infty} \sum_{n=-\infty}^{\infty} a_{l, m, n} \mathrm{e}^{\mathrm{i}(m \alpha(x-c t)+n \beta y)} T_{l}(z)\left(1-z^{2}\right)^{2},
\end{aligned}
$$

with a similar relationship holding for $\psi$. It is thus sufficient to solve for amplitude coefficients $a_{l, m, n}, b_{l, m, n}$ for $m \geqslant 0$, with the remaining coefficients obtained using the relationships $a_{l, m, n}^{R}=a_{l,-m,-n}^{R}, a_{l,-m,-n}^{I}=-a_{l, m, n}^{I}$, with corresponding expressions for $b_{l, m, n}$. The reality condition also requires the coefficients $C_{k}$ to be real. The unknown wavespeed $c$ must also be solved for, where the number of unknowns and equations is balanced by imposing one further condition to fix the phase of the wave. In the present study it is convenient to impose the condition that the imaginary part of one of the $a_{l, m, n}$ coefficients is zero (specifically we set $a_{1,2,0}^{I} \equiv 0$ ), thereby also reducing both the number of unknowns, and the number of equations that must be solved, by one. For the tertiary flows bifurcating from the two-dimensional secondary flow of the form given in expressions (3.1)-(3.3), we have $(\alpha, \beta)=(d, \grave{\beta})$ for solutions that arise due to a spanwise-superharmonic instability of the secondary flow, and $(\alpha, \beta)=(d, \grave{\beta} / 2)$ for solutions that arise due to a spanwise-subharmonic instability of the secondary flow. For the (three-dimensional) secondary flows bifurcating directly from the basic state that are considered in $\$ 5.3, \alpha$ and $\beta$ are instead the streamwise and spanwise wavenumbers of the linear disturbance to the basic flow, respectively.

Applying the Fourier operator

$$
\frac{\alpha \beta}{4 \pi^{2}} \int_{0}^{2 \pi / \beta} \int_{0}^{2 \pi / \alpha} \mathrm{e}^{\mathrm{i}(\mu \alpha(x-c t)+\gamma \beta y)} \cdot \mathrm{d} x \mathrm{~d} y
$$


to (2.8) and (2.9) we obtain nonlinear algebraic equations for $a_{l, m, n}, b_{l, m, n}, C_{k}$ and $c$, with coefficients that are functions of $z$. Numerical solutions for these unknowns can then be obtained as described in $\S 4.1$ by truncating the infinite series in (4.1)-(4.3), and evaluating these equations along with (2.10) at collocation points in the $z$ direction. In practice, for all of the solutions considered in this study, symmetry considerations further reduce the number of equations that must be solved so that it is also sufficient to only consider nonnegative values of $n$. The particular symmetry groups are discussed further in $\S 5$, and are provided in appendix A.

\subsection{Numerical method}

In order to obtain numerical solutions for the unknown amplitude coefficients $a_{l, m, n}$, $b_{l, m, n}$ and $C_{k}$, and wavespeed $c$, the infinite series in expressions (4.1)-(4.3) are truncated to corresponding finite series, $\{l, k \in \mathbb{Z}: 0 \leqslant l, k \leqslant L\},\{m \in \mathbb{Z}:-M \leqslant m \leqslant$ $M\},\{n \in \mathbb{Z}:-N \leqslant n \leqslant N\}$. Allowing $\gamma$ in operator (4.5) to take integer values from 0 to $N$ and $\mu$ integer values from 0 to $M$ (recall that reality and symmetry conditions ensure that it is sufficient to solve for the coefficients $a_{l, m, n}$ and $b_{l, m, n}$ with $m, n \geqslant 0$ ), and evaluating the resultant equations at the $L+1$ internal collocation points given by

$$
z_{i}=\cos \frac{i \pi}{L+2}, \quad i=1, \ldots, L+1,
$$

we derive nonlinear algebraic equations in the form

$$
D_{n m} X_{m}+H_{n m k} X_{m} X_{k}=0 \text {, }
$$

where the summation convention has been used, which are solved using the Newton-Raphson iteration method. The solution of the resultant linear algebraic system that must be solved at each step of the iteration is accomplished using an LU decomposition approach implemented in the Lapack routine DGESV. Generally the level of truncation (i.e. the size of $L, M, N)$ required to achieve convergence depends on the structure of the solution (the greater the variation of the solution in space the higher the truncation level required to ensure convergence), the distance from the bifurcation point (the truncation level necessary to ensure convergence generally increases with movement away from the bifurcation point) and the Reynolds number ( $L$, in particular, must be increased as the rate of shear increases with increasing $R$ ).

In order to make efficient use of computational resources we adopted an adaptive truncation level strategy in the present study. In this strategy, the truncation level is initially fixed at a level known to be sufficient to achieve convergence in a neighbourhood of the bifurcation point. This initial level can be determined from the truncation level that is needed to determine the bifurcation point to the desired accuracy in the stability analysis; the levels $(29,8,8)$ and $(29,8,16)$ are normally sufficient for superharmonic and subharmonic solutions, respectively, bifurcating at $O\left(10^{2}\right)$ values of $R$ for example. Thereafter, as the homotopy proceeds with one or more of the physical parameters $R, \Omega, \alpha, \beta$ varying in small steps, then for each solution that converges under the Newton iteration we measure the convergence in the three spatial directions using

$$
\lambda_{x}=\frac{\mathscr{P}_{x}}{\mathscr{P}}, \quad \lambda_{y}=\frac{\mathscr{P}_{y}}{\mathscr{P}}, \quad \lambda_{z}=\frac{\mathscr{P}_{z}}{\mathscr{P}},
$$

where

$$
\mathscr{P}_{x}=\max _{m \in\{M, M-1\}}\left|a_{l, m, n}^{R}\right|, \quad \mathscr{P}_{y}=\max _{n \in\{N, N-1\}}\left|a_{l, m, n}^{R}\right|, \quad \mathscr{P}_{x}=\max _{l \in\{L, L-1\}}\left|a_{l, m, n}^{R}\right|,
$$


and

$$
\mathscr{P}=\max _{l \in\{0, \ldots, L\}, m \in\{0, \ldots, M\}, n \in\{0, \ldots, N\}}\left|a_{l, m, n}^{R}\right| .
$$

If all three of the inequalities

$$
\left(\lambda_{x}, \lambda_{y}, \lambda_{z}\right) \leqslant\left(\epsilon_{x}, \epsilon_{y}, \epsilon_{z}\right)
$$

are satisfied computation proceeds with no change to the existing truncation level. Otherwise, the truncation level is increased in the direction(s) for which the inequalities (4.11) are not satisfied until they are; or the calculation is suspended if these inequalities remain unsatisfied after a reasonable number of increases, or if the solution fails to converge at a higher truncation level. We typically used $\left(\epsilon_{x}, \epsilon_{y}, \epsilon_{z}\right)=\left(10^{-5}, 10^{-5}, 10^{-7}\right)$ (experience has suggested the convergence criterion needs to be stricter in the $z$ direction projected onto modified Chebyshev polynomials than in the streamwise and spanwise directions, in which the solution is projected in a Fourier decomposition, in order to avoid numerical artefacts). Such a variable truncation level approach attempts to ensure an efficient use of computational resources while seeking to preserve accuracy across the length of the bifurcation curve. (The truncation levels quoted for the bifurcation curves in figures 4, 7, 8 and 11 refer to the maximum truncation level used along the particular bifurcation curve.) A final check on convergence and accuracy is then provided by evaluating the solution at a much higher level of truncation in all three directions at a few selected points along each solution branch. Denoting the full solution vector containing the $a_{l, m, n}, b_{l, m, n}$ and $C_{k}$ coefficients, along with the wavespeed $c$, at the higher truncation level by $\boldsymbol{x}_{\boldsymbol{F}}$, and the lower truncation level by $\boldsymbol{x}_{C}$, then an estimate of the accuracy of the latter as a solution to the Navier-Stokes equations is provided by $\left\|x_{F}-x_{C}\right\| /\left\|x_{C}\right\|$. Values of this estimate for all of the bifurcation curves shown in figures 4,8 and 11 are provided in table 1 . It can be seen that the relative error estimated in this way varies from $O\left(10^{-3}\right)$ to $O\left(10^{-9}\right)$, with a median value of $O\left(10^{-4}\right)$.

\section{Results}

Considering first three-dimensional travelling wave tertiary flows that bifurcate from the two-dimensional secondary flow with parameters $(\grave{\beta}, \Omega)=(2.5,22.1325)$, as described in $\S 3$, we consider spanwise superharmonic (i.e. $b=0$ in expressions (3.4) and (3.5)) and spanwise subharmonic $(b=\grave{\beta} / 2)$ secondary disturbances, which correspond to wavenumber pairs $(\alpha, \beta)=(d, \grave{\beta})$ and $(\alpha, \beta)=(d, \grave{\beta} / 2)$, respectively, for the tertiary flow. Possible bifurcation points for such tertiary flows are given by neutral stability points at which $\sigma^{R}=0$, where $\sigma^{R}$ denotes the real part of $\sigma$ appearing in expressions (3.4) and (3.5). The first stage in obtaining such three-dimensional flows thus lies in analysing the stability of the secondary flows.

In figure 2 we trace the real parts of the leading-order least-stable secondary eigenmodes against varying $d$ at a point close to the turning point on the upper branch of two-dimensional secondary flow for superharmonic and subharmonic secondary disturbances. In addition to the result that the secondary flow is always stabilized for large enough $d$, it can be observed that, although there is only ever a single mode that is unstable for a given secondary disturbance wavenumber pair $(d, b)$, there are two different eigenmodes that describe this unstable mode, one for $d=O\left(10^{-1}\right)$, one for $d=O(1)$. (In fact, it can be seen that there are three different modes that describe the most unstable mode as $d$ increases from zero, but the second of these is always stable, i.e. always has a strictly negative growth rate). Different secondary eigenmodes 


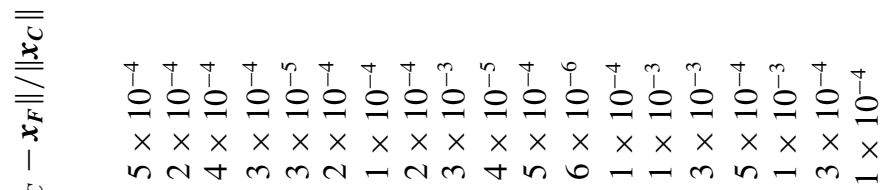

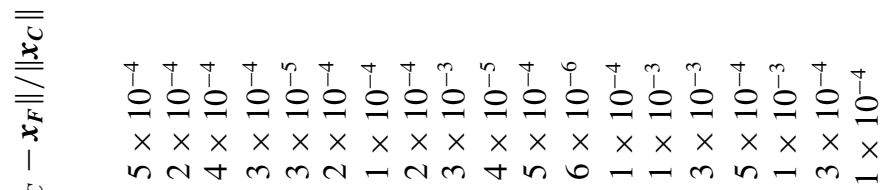

$\stackrel{\circlearrowright}{=}$

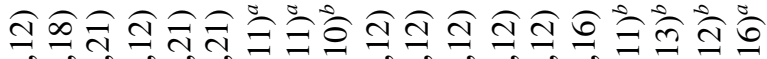

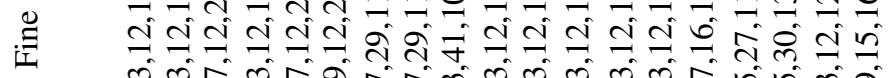

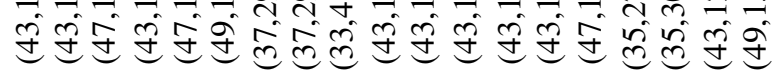

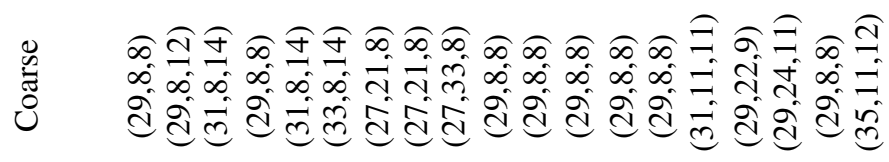

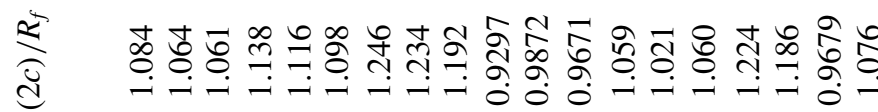

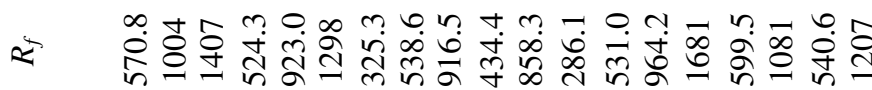

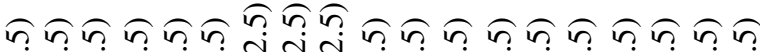

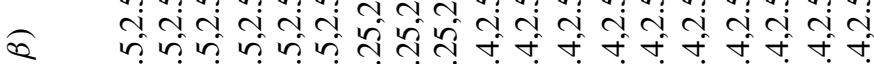

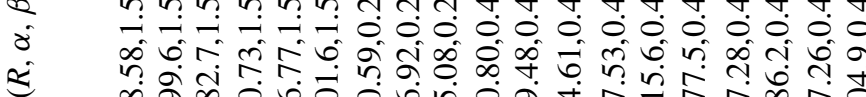

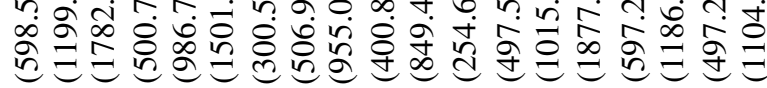

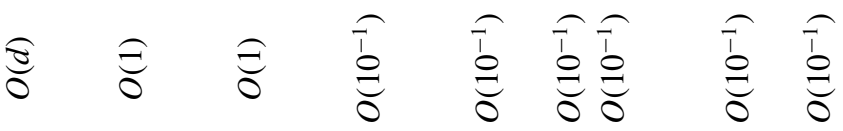

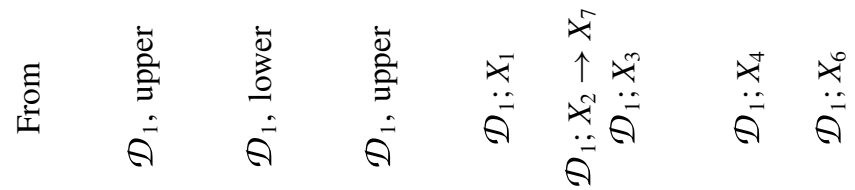

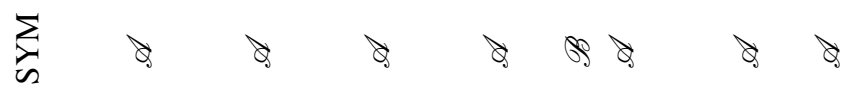

$\sum_{2}^{\circledR}$

竞 
Three-dimensional exact coherent states in rotating channel flow

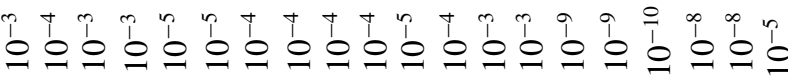

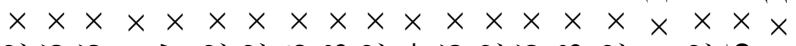

$\stackrel{\text { ֻׁ }}{=}$

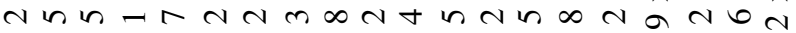

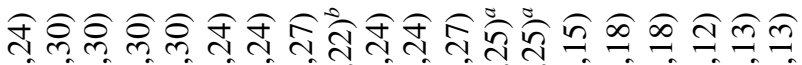

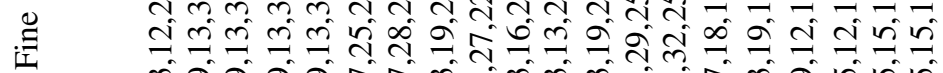

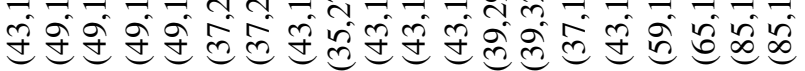

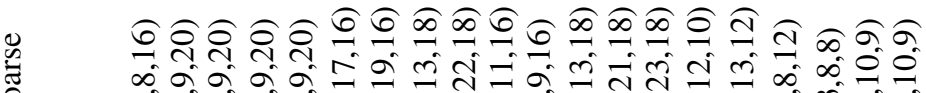

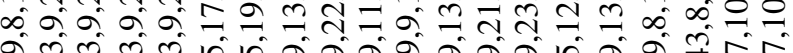
वेल्व

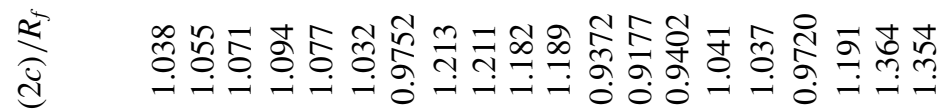

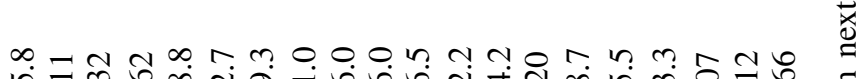

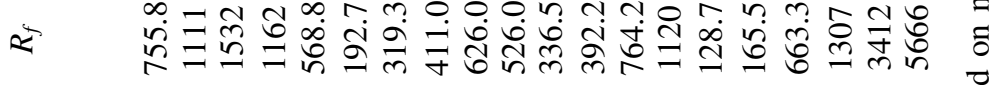

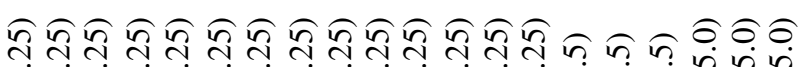

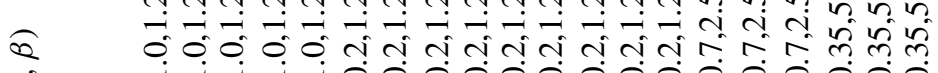

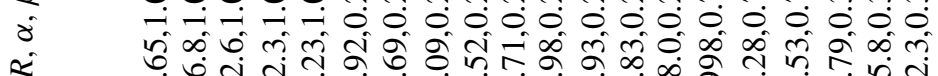

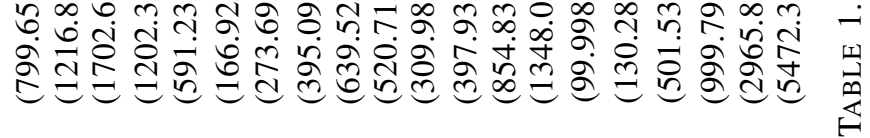

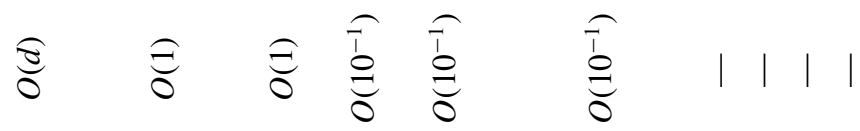

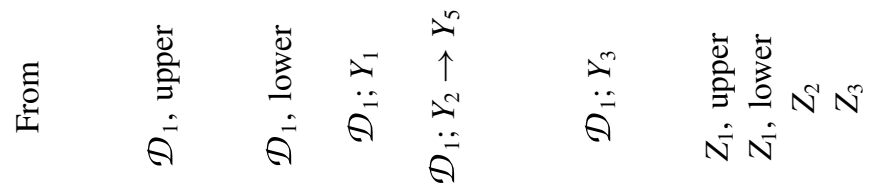

$\sum$ b b b b b b be be be

$\stackrel{Ð}{2}$

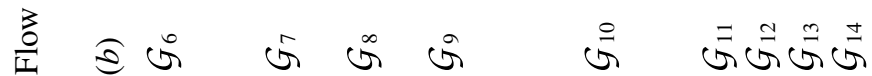




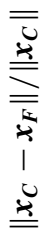

으의의

$\times \times \times \times \times \times$

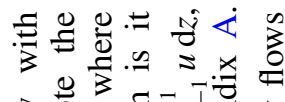

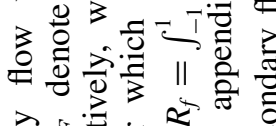

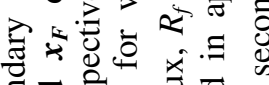

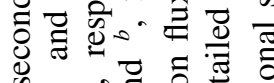

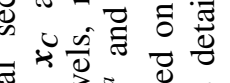

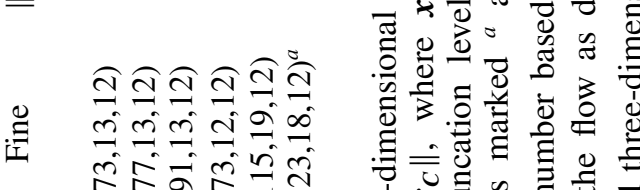

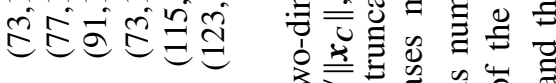

引三

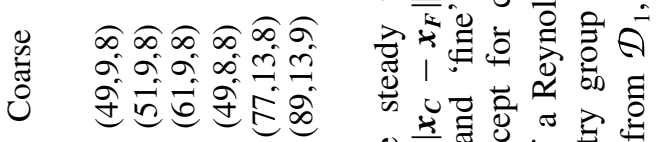

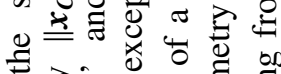

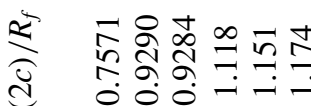

तें

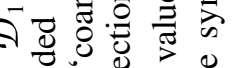

:

氙

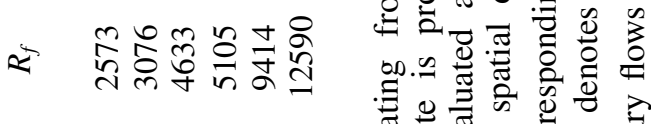

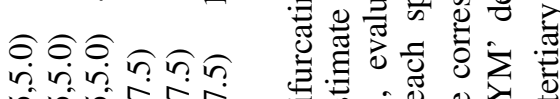

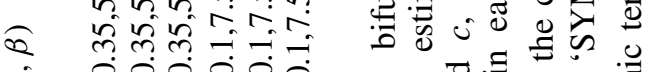

\& 000000

- $\quad$ ते

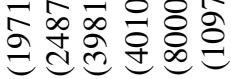

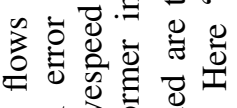

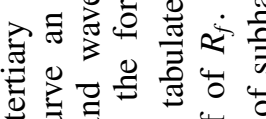

ఠ

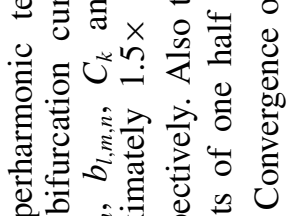

竞 N

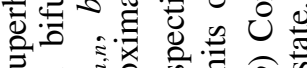
के पै

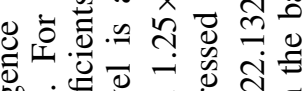

$\sum_{i=1}$ bo

总

高 G G G อั்่ ป⿰讠仑 ฮิ่ 저월

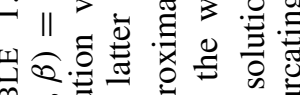

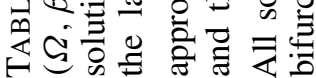



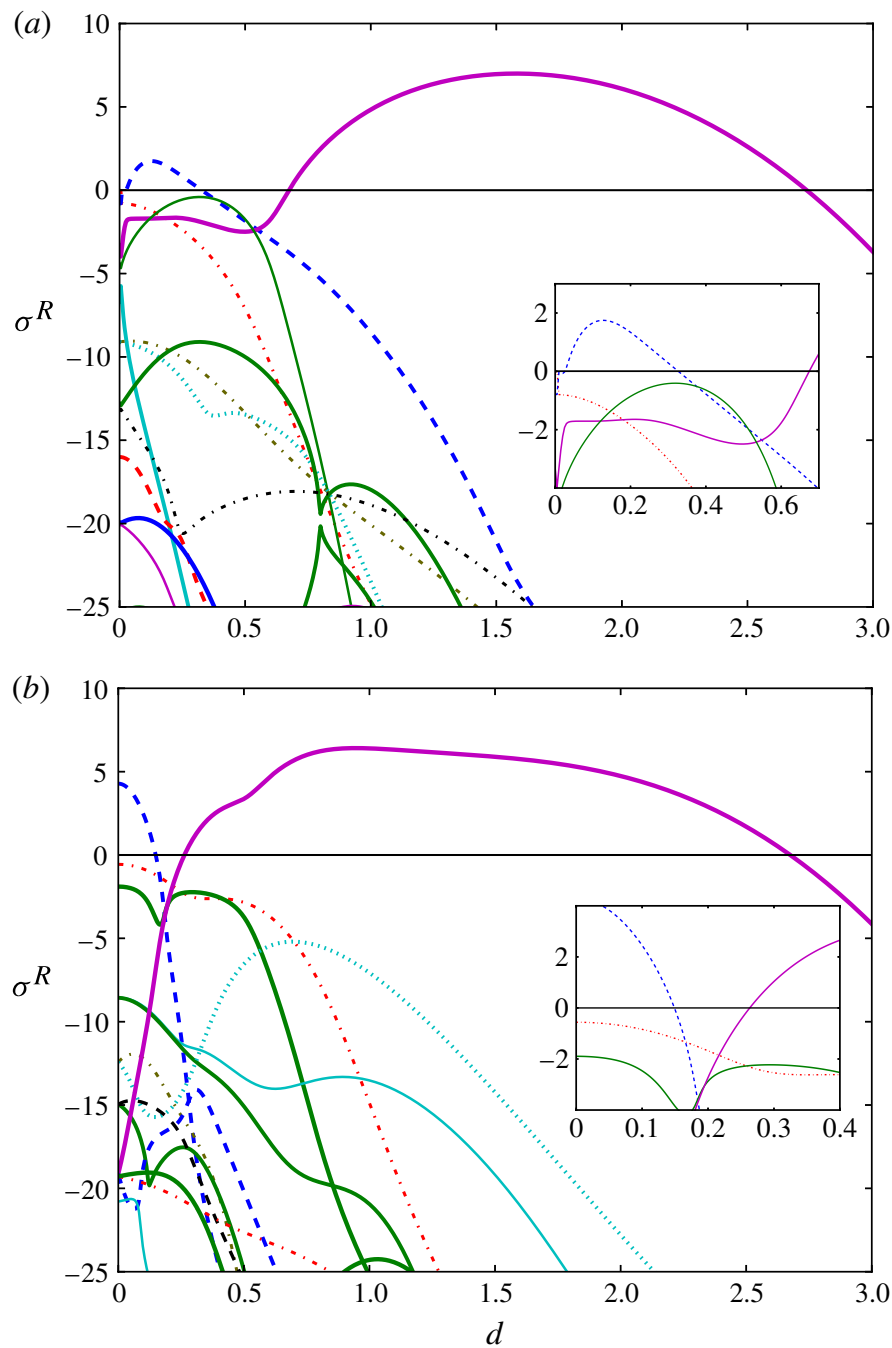

FIGURE 2. (Colour online) Stability of the streamwise-independent secondary flow: dependence of the leading-order least-stable eigenmodes on the streamwise secondary disturbance wavenumber parameter, $d$. Two distinct different modes become unstable, one for $d=O\left(10^{-1}\right)$ and one for $d=O(1)$. Calculations for the flow with $(R, \beta, \Omega)=$ $(374.1,2.5,22.1325)$ on the upper solution branch subject to superharmonic $(a)$ and subharmonic $(b)$ disturbances, for $(L, N)=(23,7)$.

would be expected to yield different nonlinear tertiary flows even if the wavenumber parameters and symmetry of the bifurcating flows are the same (the eigenfunction of the secondary mode determines the spatial structure of the bifurcating flow), and so we must consider the bifurcations of each of these modes in order to give a complete picture of the possible tertiary flows.

Further understanding is gained by fixing $d$ at $O\left(10^{-1}\right)$ and $O(1)$ values, and analysing the behaviour of the secondary stability eigenmodes with changes in $R$. In figure 3 we trace the real $\left(\sigma^{R}\right)$ parts of the most unstable secondary eigenmodes for spanwise superharmonic and subharmonic secondary disturbances with fixed values 

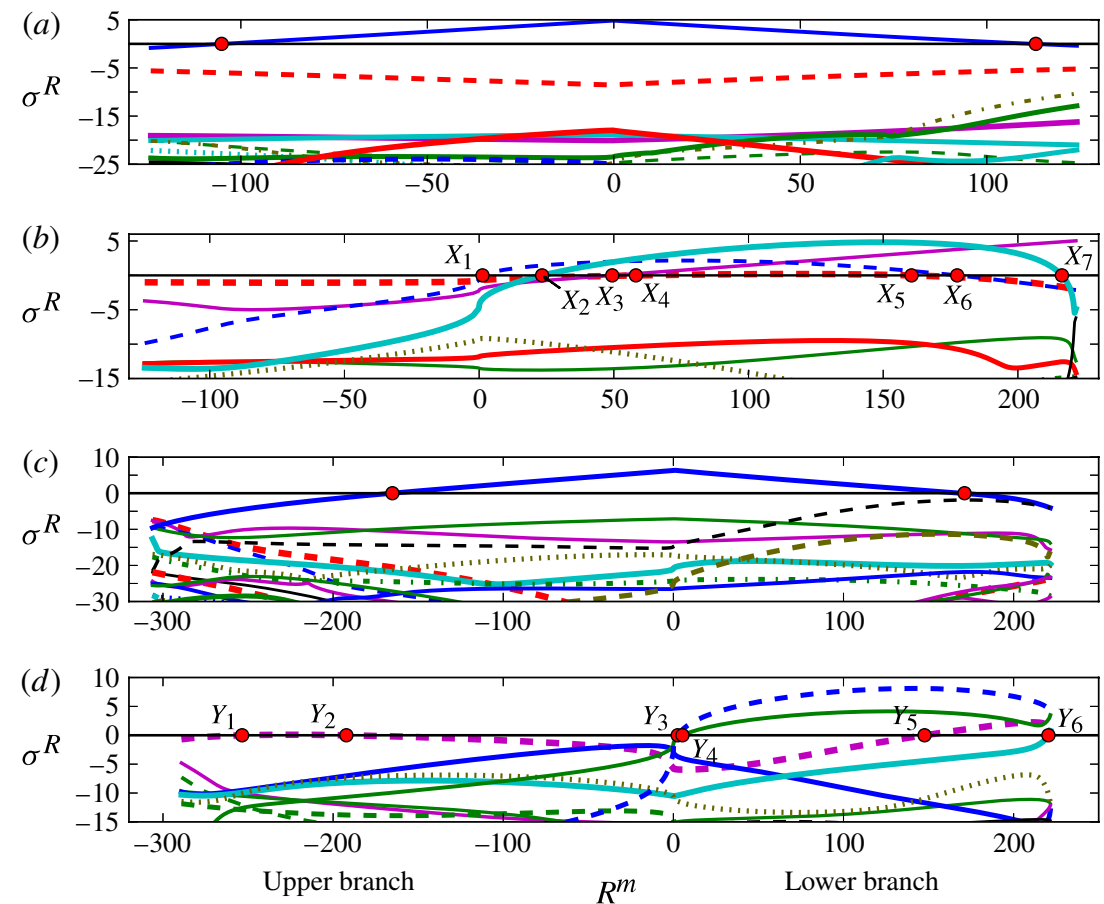

FIGURE 3. (Colour online) Stability of the secondary flow against $R$, and identification of bifurcation points $(-)$ for the tertiary flow. Only a single mode can become unstable when $d=O(1)$, while for $d=O\left(10^{-1}\right)$ up to four distinct modes can become unstable. Real parts of the least-stable eigenmodes for the secondary flow with $(\beta, \Omega)=(2.5,22.1325)$ against varying $R^{m}$, where $R^{m}=\left(R-R^{T P}\right)$ along the upper, and $\left(R^{T P}-R\right)$ along the lower branch of the secondary flow, where $R^{T P}=374.1$ denotes the value of $R$ at the turning point of this flow. Superharmonic $(a)(d, b)=(1.0,0),(b)(d, b)=(0.4,0)$; subharmonic $(c)$ $(d, b)=(1.0,1.25),(d)(d, b)=(0.2,1.25)$; with $(L, N)=(23,7)$.

of $d$ around the upper and lower branches of the secondary flow. It can be seen that we have further that for $d=O(1)$, the secondary flow becomes unstable only to a single complex mode, with this mode becoming unstable on the upper branch of the secondary flow and restabilizing on the lower branch. For $d=O\left(10^{-1}\right)$, however, while only a single eigenmode becomes unstable on the upper branch, the secondary flow may become unstable to up to four distinct eigenmodes on the lower branch.

More precisely we will define the $d=O\left(10^{-1}\right)$ regime to be given by the domain in which the maximum possible number of eigenmodes is unstable, while the $d=O(1)$ regime is the domain in which the single mode described above is unstable. For a range of $\grave{\beta}$ values, approximate values for the upper and lower boundaries of the $d=O\left(10^{-1}\right)$ and $d=O(1)$ regimes, respectively, are listed in table 2 (note the superharmonic regime as described above does not exist for $\grave{\beta}=2.0$ ). The two regimes are in general disjoint, but since there are no eigenmodes appearing outside these two regime domains that are not included in one or both of the regimes, a consideration of the two regimes is sufficient to describe all of the modes. Table 2 will also describe the domain of existence in the streamwise wavenumber $\alpha$ of the bifurcating tertiary flows in a neighbourhood of the bifurcation point, but these domains will vary with movement away from the bifurcation point, and an overlapping or exchange of the 


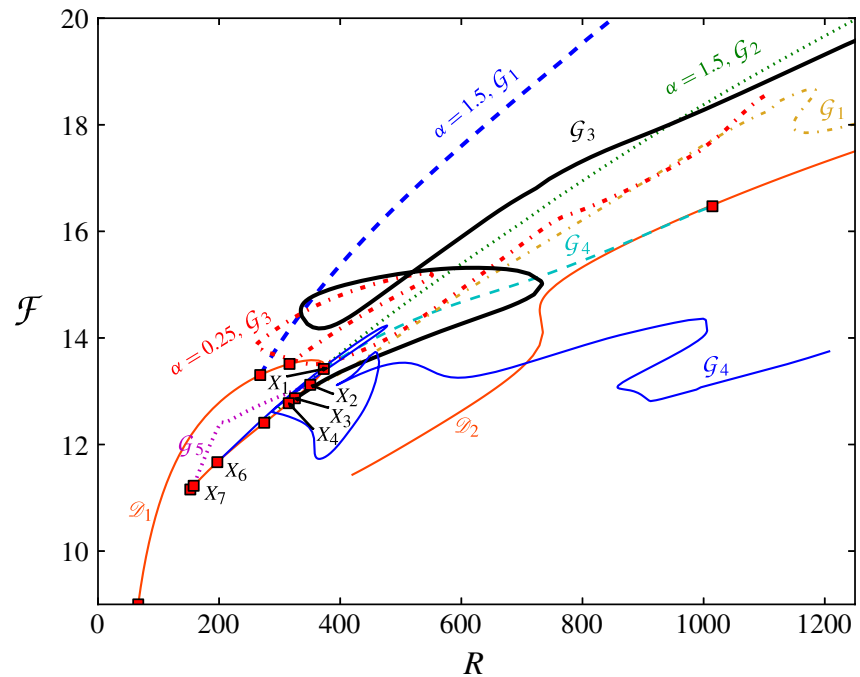

FIGURE 4. (Colour online) Bifurcation of the superharmonic three-dimensional travelling wave tertiary flows from the secondary flow $\mathcal{D}_{1}$; friction factor $\mathcal{F}=R \lambda$ plotted against $R$ for fixed $\Omega=22.1325$. All tertiary flow solutions have $\beta=2.5$, and $\alpha=0.4$ unless otherwise labelled. The rich structure of solutions to the Navier-Stokes equations can be observed; for example, the plot shows 10 flows with parameters $(\alpha, \beta)=(0.4,2.5)$ at $R=400$. Bifurcation points are marked by squares, and the $\alpha=0.4$ lower-branch bifurcation points are additionally labelled according to figure 3. The following truncation levels $(L, M, N)$ were used: $\alpha=0.25$, upper $(27,21,8) ; X_{3},(31,11,11) ; X_{4},(29,14,9) ; X_{6},(35,11,14)$, with $(29,8,8)$ used for all other three-dimensional flows, and levels $(15,0,7)$ and $(41,0,21)$, respectively, for the two-dimensional solutions $\mathcal{D}_{1}$ and $\mathcal{D}_{2}$.

\begin{tabular}{lcccccc}
\multicolumn{4}{c}{ Superharmonic } & \multicolumn{3}{c}{ Subharmonic } \\
$\dot{\beta}$ & $\mathscr{N}$ & $O\left(10^{-1}\right)$ & $O(1)$ & $\mathscr{N}$ & $O\left(10^{-1}\right)$ & $O(1)$ \\
2.0 & 3 & $d<0.1$ & - & 2 & $d<0.5$ & - \\
2.25 & 4 & $d<0.3$ & $d>0.7$ & 3 & $d<0.2$ & $d>0.8$ \\
2.5 & 4 & $d<0.4$ & $d>0.7$ & 4 & $d<0.3$ & $d>0.9$ \\
2.7 & 4 & $d<0.5$ & $d>0.8$ & 4 & $d<0.2$ & $d>1.0$ \\
3.0 & 4 & $d<0.7$ & $d>0.9$ & 4 & $d<0.3$ & $d>1.0$ \\
3.5 & 4 & $d<0.7$ & $d>1.0$ & 5 & $d<0.1$ & $d>1.1$ \\
4.0 & 4 & $d<0.4$ & $d>1.5$ & 5 & $d<0.2$ & $d>1.3$
\end{tabular}

TABLE 2. Approximate values for the upper boundary of the $d=O\left(10^{-1}\right)$ regime, in which $\mathscr{N}$ eigenmodes can be concurrently unstable, and the lower boundary of the $d=O(1)$ regime, in which a single eigenmode is unstable, for secondary flows bifurcating from the basic flow for the values of $\grave{\beta}$ indicated when $\Omega=22.1325$.

two domains may also be possible. An investigation of such phenomena is beyond the scope of the current study. As stated above, this study principally focuses on the $\grave{\beta}=2.5$ secondary flow, which bifurcates from close to the linear critical point. With reference to table 2 , the only mode that is thereby excluded from consideration is the fifth least-stable mode, which becomes unstable for subharmonic disturbances 
when $\grave{\beta}>3$. For completeness we attempted to also find tertiary flows bifurcating from points at which this mode changes stability when $\grave{\beta}=3.5$. However, all attempts ended with the Newton-Raphson iteration yielding either no converged solution or the two-dimensional secondary flow. Furthermore, as detailed in $\$ \S 5.1$ and 5.2, for the $\grave{\beta}=2.5$ flow we were unable to find flows bifurcating from one of the possible superharmonic bifurcation points, and from two of the possible subharmonic points. With the exception of these modes however, the present analysis for $\grave{\beta}=2.5$ thus provides a qualitative description of all of the tertiary flows bifurcating from secondary flows in the range $2 \leqslant \grave{\beta} \leqslant 4$. Note in particular that this range includes the interval $(2.35 \leqslant \grave{\beta} \leqslant 3.45)$ of stable secondary flows (see Wall \& Nagata 2006, figure 12).

Thus, given the discussion above, in order to present a complete description of the tertiary flows bifurcating from the secondary flow, we should consider these two magnitudes of $d$, i.e. $d=O(1)$ and $d=O\left(10^{-1}\right)$, for the two values of $b=0, \grave{\beta} / 2$. Henceforth, three-dimensional tertiary flows arising upon a loss of stability of the twodimensional secondary flow to a superharmonic (subharmonic) mode will be described as 'superharmonic' ('subharmonic') tertiary flows. A consideration of the symmetry of the amplitude coefficients of the bifurcating flows finds that two symmetry groups are possible for the bifurcating superharmonic flows, listed in appendix A as groups $\mathscr{A}$ and $\mathscr{B}$. The form of the eigenvector of the neutrally stable eigenmode at the bifurcation point determines which of these groups is selected. For the subharmonic flows only one symmetry group is possible, listed as $\mathscr{C}$ in appendix A. It is straightforward to show that, in particular, the group $\mathscr{A}$ solutions satisfy the glidereflectional symmetry (glide half a wavelength in the streamwise direction, reflection in the plane $y=\pi / \beta$ )

$$
\left(\begin{array}{c}
u \\
v \\
w
\end{array}\right)\left(x+\frac{\pi}{\alpha}, \frac{2 \pi}{\beta}-y, z\right)=\left(\begin{array}{c}
u \\
-v \\
w
\end{array}\right)(x, y, z)
$$

and the group $\mathscr{B}$ solutions satisfy the reflectional symmetry (reflection about the plane $y=\pi / \beta)$

$$
\left(\begin{array}{c}
u \\
v \\
w
\end{array}\right)\left(x, \frac{2 \pi}{\beta}-y, z\right)=\left(\begin{array}{c}
u \\
-v \\
w
\end{array}\right)(x, y, z),
$$

while the group $\mathscr{C}$ solutions satisfy the glide-reflectional symmetry (glide half a wavelength in the streamwise direction, reflection about the plane $y=\pi /(2 \beta))$

$$
\left(\begin{array}{c}
u \\
v \\
w
\end{array}\right)\left(x+\frac{\pi}{\alpha}, \frac{\pi}{\beta}-y, z\right)=\left(\begin{array}{c}
u \\
-v \\
w
\end{array}\right)(x, y, z)
$$

and also satisfy the reflective symmetry (reflection about the line $y=\pi / \beta$ ) satisfied by the group $\mathscr{B}$ solutions, and thereby also satisfy the composition of transformations 
(5.2) and (5.3), that is the double-glide symmetry

$$
\left(\begin{array}{c}
u \\
v \\
w
\end{array}\right)\left(x+\frac{\pi}{\alpha}, y+\frac{\pi}{\beta}, z\right)=\left(\begin{array}{c}
u \\
v \\
w
\end{array}\right)(x, y, z) .
$$

Symmetry alone, however, is insufficient to fully distinguish between different flow types. For example, all of the flows shown in figures 9 and 12 are of symmetry group $\mathscr{C}$, but clearly all exhibit distinct flow structures. Noting that all of the flows of the present study are found in bifurcations from either the two-dimensional secondary flow (in the case of the three-dimensional tertiary flows) or from the basic flow (in the case of the three-dimensional secondary flows), in order to objectively identify between different flow types we distinguish on the basis of: (i) symmetry; (ii) originating eigenmode(s); and (iii) major solution branches. If one or more of these three properties are different between two solutions then we classify the solutions as distinct. Regarding (iii), we designate a 'major' solution branch to be one of two branches of a bifurcation curve that connects (i.e. bifurcates from) two different flows and features at least one turning point. In practice all such tertiary flows reported in this study bifurcate from the upper and/or lower branches of the secondary flow. It is shown below that distinguishing flows on this basis allows us to identify 15 distinct three-dimensional travelling wave flows: 5 superharmonic tertiary flows, $\mathcal{G}_{1}, \ldots, \mathcal{G}_{5}$, 5 subharmonic tertiary flows, $\mathcal{G}_{6}, \ldots, \mathcal{G}_{10}$, and 5 secondary flows, $\mathcal{G}_{11}, \ldots, \mathcal{G}_{15}$.

In $\S \S 5.1$ and 5.2 below superharmonic and subharmonic three-dimensional tertiary flows for $d=O(1)$ and $d=O\left(10^{-1}\right)$, are discussed and their flow structures are visualized, while in $\$ 5.3$ we consider the three-dimensional secondary flows that bifurcate directly from the basic state. In the visualizations we plot the streamwise component of velocity, $u$, and the streamwise component of vorticity, $\omega_{1}=[\nabla \times \boldsymbol{u}] \cdot \boldsymbol{i}=$ $\boldsymbol{\omega} \cdot \boldsymbol{i}$, where all of the visualization plots are shown plotted in the scaled spanwise and streamwise coordinates $\left(x^{\prime}, y^{\prime}\right)=(1 / 2 \pi)(\alpha x, \beta y)$.

\subsection{Superharmonic tertiary flows}

Distinguishing on the basis of symmetry, originating eigenmode and major solution branches as described above, we identify a total of five distinct superharmonic flows.

$d=O(1)$

One distinct superharmonic flow type, $\mathcal{G}_{1}$, is given by the flow bifurcating from the upper branch of the secondary flow. The bifurcation curve for such a superharmonic tertiary flow with $(\alpha, \beta)=(1.5,2.5)$ that arises in a bifurcation at $R=268$ due to a change in stability of the least-stable eigenmode is shown in figure 4 . Here the scaled friction factor $\mathcal{F}=R \lambda$ is used as a measure of the amplitude of the bifurcating solution, in which

$$
\lambda=\frac{2 L J}{\rho u_{m}^{* 2}}=\frac{16 R}{\left(\int_{-1}^{1} u \mathrm{~d} z\right)^{2}}
$$

is the friction factor, where $u_{m}^{*}=(1 / 2 L) \int_{-L}^{L} u^{*} \mathrm{~d} z$. The corresponding plots for the flux-based Reynolds number $R_{f}$ are similar and are provided in figure 22 in the supplementary material available at http://dx.doi.org/10.1017/jfm.2013.242. It can be seen that the solution increases steadily with $R$, and no turning point has been found 

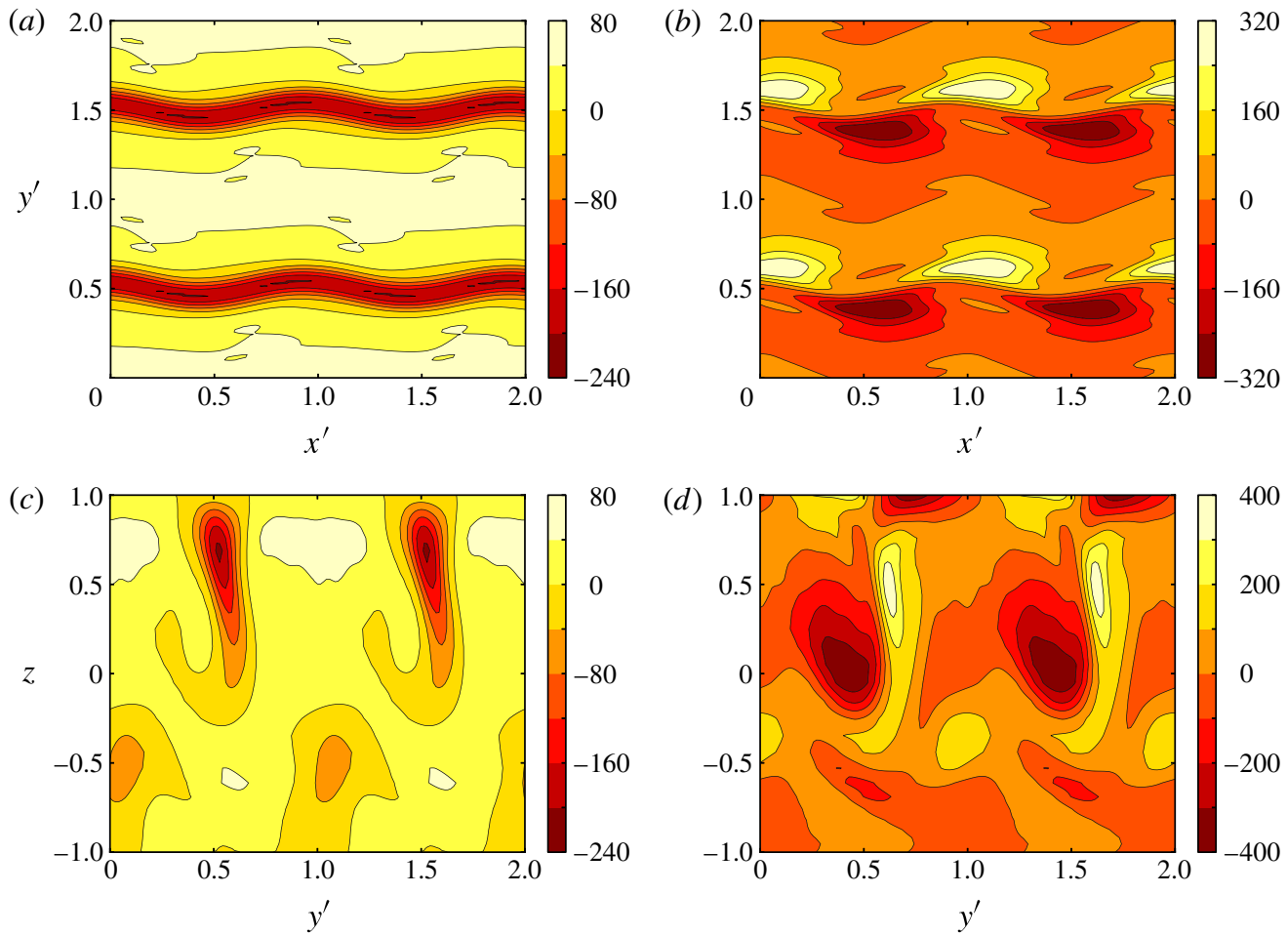

FIGURE 5. (Colour online) Visualization of $(a, c) u$ and $(b, d) \omega_{1}$ for the superharmonic $\mathcal{G}_{1}$ flow. A single sinusoidal low-speed streak per spanwise wavelength is flanked by staggered, streamwise-orientated vortices. Parameter values are $(R, \alpha, \beta, \Omega)=(800,1.5,2.5,22.1325)$ calculated at $(L, M, N)=(29,8,8)$. Plots $(a, b)$ show the solution in a streamwise-spanwise plane at $z=0.612$, plots $(c, d)$ show the solution in a spanwise- wall normal plane at $x^{\prime}=0$. Two wavelengths are shown in the scaled variables $\left(x^{\prime}, y^{\prime}\right)=(1 / 2 \pi)(\alpha x, \beta y)$.

as far as the solution has been tracked, up to $R=1800$. This flow produces the largest values of $\mathcal{F}$ found for the superharmonic flows for values of $R>357$.

The structure of this flow at $R=800$ is visualized in figure 5. It can be seen that this flow is distinguished by low-speed sinusoidal streaks in $u$ that are orientated in the streamwise direction, and are flanked by staggered vortices that are also approximately orientated in the streamwise direction. There is one such low-speed streak per spanwise wavelength. As was found for the two-dimensional flows described by Wall \& Nagata (2006), it is also the case for the present three-dimensional flows that the fluctuating flow structures are largely concentrated in the half of the channel $(0 \leqslant z \leqslant 1)$ that is unstable according to Pedley's (1969) inviscid stability criterion. An animation of the velocity vectors in the spanwise-wall-normal plane is provided in supplementary movie 1 . It can be seen from the animation that the two vortices that accompany a given low-speed streak follow a cycle characterized by a vertical and sideways motion of the vortex centres, and in which one vortex is strongest when the other is weakest. For this flow, the vertical motion is larger than the sideways motion.

A second superharmonic flow, $\mathcal{G}_{2}$, is found by considering the flow bifurcating from the lower branch of the secondary flow. In particular, we consider here the flow with $(\alpha, \beta)=(1.5,2.5)$ that arises in a bifurcation at $R=274$. It can be seen with reference to figure 6 that, in contrast with the upper-branch solution, the flow 

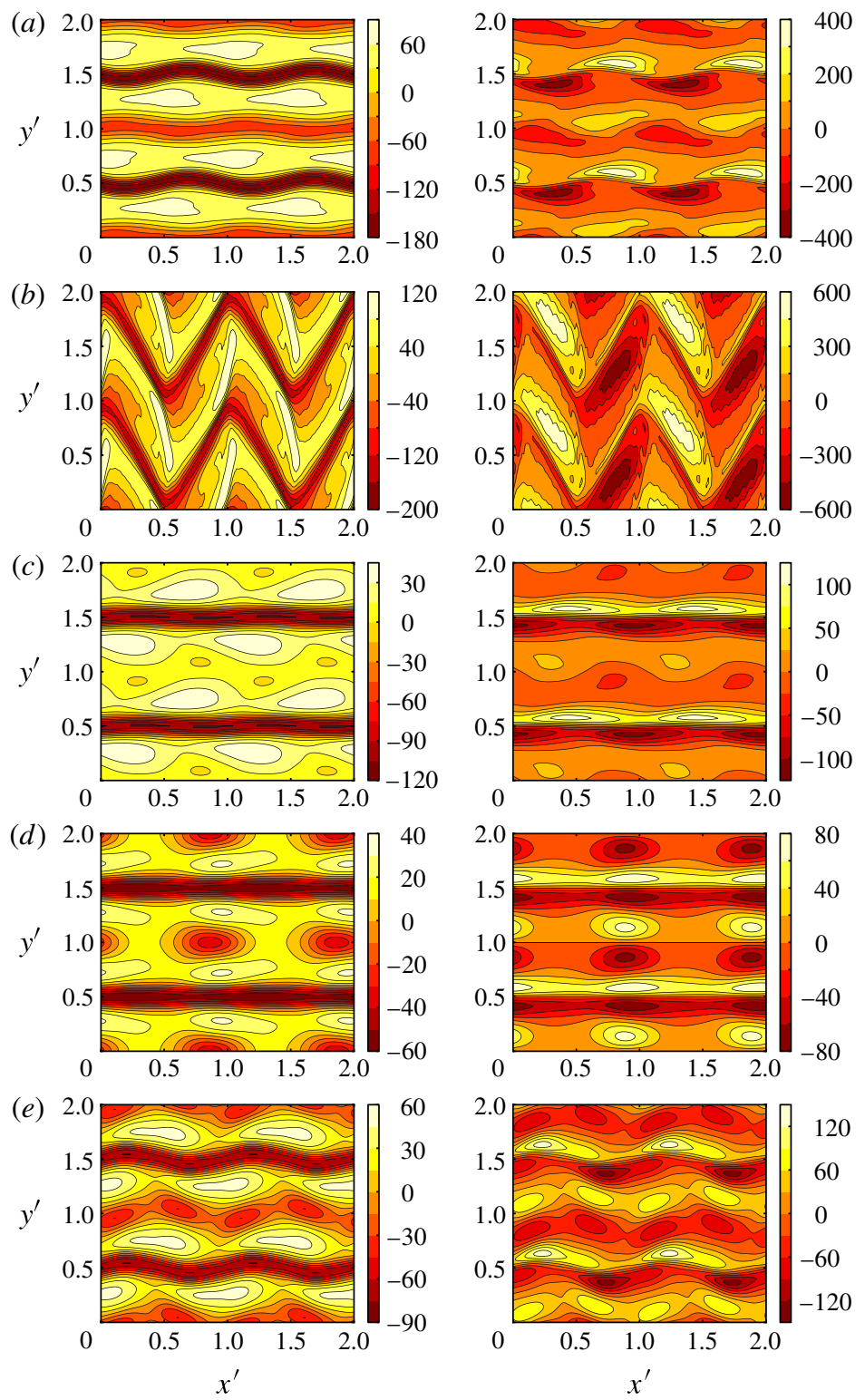

FIGURE 6. (Colour online) Visualization of (left) $u$ and (right) $\omega_{1}$ of superharmonic tertiary flows in a streamwise-spanwise plane. Sinusoidal low-speed streaks are always flanked by staggered vortices, low-speed pulses and varicose streaks are flanked by aligned vortices. Flows bifurcating from: $(a)\left(\mathcal{G}_{2}\right)$ lower branch when $d=O(1),(R, \alpha)=(800,1.5)$ at $z=0.612 ;(b) \quad\left(G_{3}\right)$ upper branch when $d=O\left(10^{-1}\right),(R, \alpha)=(800,0.25)$ with $(L, M, N)=(27,21,8)$ at $z=0.561 ;(c)\left(\mathcal{G}_{4}\right)$ lower branch when $d=O\left(10^{-1}\right),\left(X_{1}\right.$ in figure 3$),(R, \alpha)=(449,0.4)$ at $z=0.689 ;(d)\left(\mathcal{G}_{5}\right)$ lower branch when $d=O\left(10^{-1}\right),\left(X_{2}\right.$ in figure 3$),(R, \alpha)=(268,0.4)$ at $z=0.689$; $(e)\left(\mathcal{G}_{4}\right)$ lower branch when $d=O\left(10^{-1}\right)$, $\left(X_{6}\right.$ in figure 3$),(R, \alpha)=(356,0.4)$ at $z=0.689$. All flows have $(\beta, \Omega)=(2.5,22.1325)$, and $(L, M, N)=(29,8,8)$ unless stated otherwise. Two wavelengths are shown in the scaled variables $\left(x^{\prime}, y^{\prime}\right)=(1 / 2 \pi)(\alpha x, \beta y)$. 
structure features two low-speed streaks per spanwise wavelength. One of the streaks is, however, much weaker and smaller than the other (see, for example, the streaks located at $y^{\prime}=0,1$ and 2 in the plots), and is located closer to the $z=1$ wall (not shown in the figure), together with its accompanying staggered vortices. Continuing the solution in the direction of increasing $R$ as shown in figure 4, once again no turning point has been found as far as the solution has been tracked, up to $R=1500$.

$d=O\left(10^{-1}\right)$

A third distinct flow, $\mathcal{G}_{3}$, is given by considering the superharmonic flow bifurcating from the upper branch when $d=O\left(10^{-1}\right.$ ) (recall that only a single eigenmode becomes unstable on the upper branch of the secondary flow). Here we consider the representative example of the solution with $\alpha=0.25$ bifurcating at $R=316$. The flow is visualized in figure 6. Similar to the flow shown in figure 5, this flow is also characterized by one low-speed streak in $u$ per spanwise wavelength, with each streak flanked by staggered vortices. However, the degree of spanwise motion of streaks is significantly greater. Indeed the degree of the spanwise motion of these streaks increases as the solution moves away from the bifurcation point until, as shown in the present plots, the streaks adopt a zig-zag profile in the spanwise-streamwise plane, with a sideways movement that fluctuates across a full spanwise wavelength. The large sideways movement of the vortices that accompany the streaks can also be seen in supplementary movie 2, which shows an animation of the velocity vectors in the spanwise-wall-normal plane. Strong vortex structures can be observed moving between both channel walls and the channel centreline $(z=0)$. With reference to figure 4 , it can be seen that the bifurcation curve for this flow also differs from those considered above, first moving away from the bifurcation point in the direction of increasing $R$ until a turning point is encountered at around $R=555$. The bifurcation curve then moves in the direction of decreasing $R$ until a second turning point is encountered at around $R=264$, whereupon it moves in the direction of increasing $R$ as far as it has been tracked (up to $R=1000$ ). As discussed in greater length in $\S 7$, this class of flow appears to correspond to the flows labelled 'WVF2' in Finlay's (1990) simulation study.

On the lower branch of the secondary flow for $d=O\left(10^{-1}\right)$, as noted previously, a total of four eigenmodes become unstable. We consider the representative case $d=0.4 \quad(b=0)$, for which the secondary stability plot is plotted in figure 3 . Given that three of the eigenmodes that lose stability on the lower branch later restabilize with further movement along this branch of the secondary flow, we therefore have a total of seven possible bifurcation points, $X_{1}, \ldots, X_{7}$ as marked in the figure, occurring at the Reynolds numbers $\left(R^{X_{1}}, R^{X_{2}}, R^{X_{3}}, R^{X_{4}}, R^{X_{5}}, R^{X_{6}}, R^{X_{7}}\right)=$ $(373,351,325,315,214,197,158)$. We were unable to find a solution bifurcating from point $X_{5}$, but solutions bifurcating from the other points are reported here.

Considering first the flow bifurcating from $X_{1}$, where the least-stable eigenmode destabilizes, we obtain the fourth distinct superharmonic tertiary flow, $\mathcal{G}_{4}$. It can be seen from figure 4 that the solution bifurcating from this point $(R=373)$ close to the turning point of the secondary flow moves in the direction of increasing $R$ before terminating on another two-dimensional flow (which does not bifurcate from the basic state), marked $\mathcal{D}_{2}$ in the figure, of the same wavenumber $(\grave{\beta}=2.5)$ as the secondary flow from whence it bifurcated. Stability analysis of this secondary flow confirms that an eigenmode (the third least stable) loses stability as $R$ decreases through $R=999$. A visualization of this flow is provided in figure 6 . It can be seen that the flow is characterized by one strong low-speed streak and one weak low-speed 
streak in $u$ per spanwise wavelength. The weak low-speed streaks are just visible at $y^{\prime}=0,1$ and 2 in the present plot. The same eigenmode that loses stability at point $X_{1}$ regains stability at point $X_{6}$ as marked in figure 3, and the structure of the flow that bifurcates from the latter point is shown in figure $6(e)$, and is clearly qualitatively similar to that bifurcating from point $X_{1}$, and so is also classed as a $\mathcal{G}_{4}$ flow. From figure 6 it can be seen more clearly that the weaker low-speed streak in fact consists of pulses of low-speed $u$ (henceforth referred to as 'pulses') occurring periodically within a sinusoidal envelope. It can be seen that these pulses are, in contrast to all of the previously described flow structures, accompanied by aligned vortices, while the stronger sinusoidal low-speed streaks in $u$ are again accompanied by staggered vortices. It can be seen from figure 4 that the bifurcation curve for the flow bifurcating from point $X_{6}$ passes through several turning points as far as the solution has been tracked (up to $R=1200$ ), with multiple solution branches existing for many values of $R$ in the solution domain. With further movement along the solution branch the stronger low-speed streaks (at $y=0.5,1.5$ in figure $6 e$ ) fragment into a sinusoidal line of low-speed pulses to yield a more complicated looking flow, see figure 16 for example.

The fifth distinct superharmonic tertiary flow, $\mathcal{G}_{5}$, is identified as that bifurcating from point $X_{2}$ in figure 3 (where the second least-stable eigenmode loses stability). With reference to figure 4 , it can be seen that this flow arises in a supercritical bifurcation at $R=351$, with the solution branch then also terminating on the same secondary flow at $R=158$ (position $X_{7}$ in figure 3) when the same eigenmode restabilizes. The flow is visualized in figure 6 . This solution selects a different symmetry group ( $\mathscr{B}$ in appendix A) from the other superharmonic solutions presented in this section. Similar to the superharmonic flow bifurcating from the upper branch, there is again one low-speed streamwise-orientated streak in $u$ per spanwise wavelength. However, in contrast to this other superharmonic flow, the streaks are varicose in nature, and are flanked by aligned vortices. We also again note the appearance of low-speed pulses in $u$ along a streamwise line precisely half a spanwise wavelength between any two adjacent low-speed streaks (i.e. at $y^{\prime}=0,1$ and 2 in the $u$ plot of figure $6 d$ ). These low-speed pulses are also flanked by aligned vortices. An animation of the velocity vectors in the spanwise-wall-normal plane for this flow is provided in supplementary movie 3, in which the vortices that flank the low-speed pulses can be observed lying close to the $z=1$ channel wall.

We have been unable to find a flow bifurcating from the point where the fourth least-stable eigenmode restabilizes ( $X_{5}$ in figure 3 ), but have found flows bifurcating from points $X_{3}$ and $X_{4}$. However, neither of these two flows contribute distinct flows to those already discussed. With reference to figure 4 , the solution bifurcating from point $X_{3}$, where the third least-stable eigenmode loses stability, moves in the direction of increasing $R$ encountering two turning points in close succession (at around $R=1190$ and $R=1150$ ), before the solution continues in the direction of increasing $R$ as far as it has been tracked, up to $R=1900$ (note that the bifurcation curve near the bifurcation point is obscured by that of the solution bifurcating from point $X_{4}$ in the figure). It can be seen, however, with reference also to figure 2 that this mode corresponds to the mode that gives rise to the upper and lower branch $d=O(1)$ solutions, and the flow structure (not shown) at first largely resembles the $\mathcal{G}_{2}$ flow shown in figure 6(a) with a weaker low-speed streak interspersing stronger low-speed streaks, before these weaker low-speed streaks vanish on passing through the two turning points, resulting in a flow that resembles the $\mathcal{G}_{1}$ flow shown in figure 5 . 

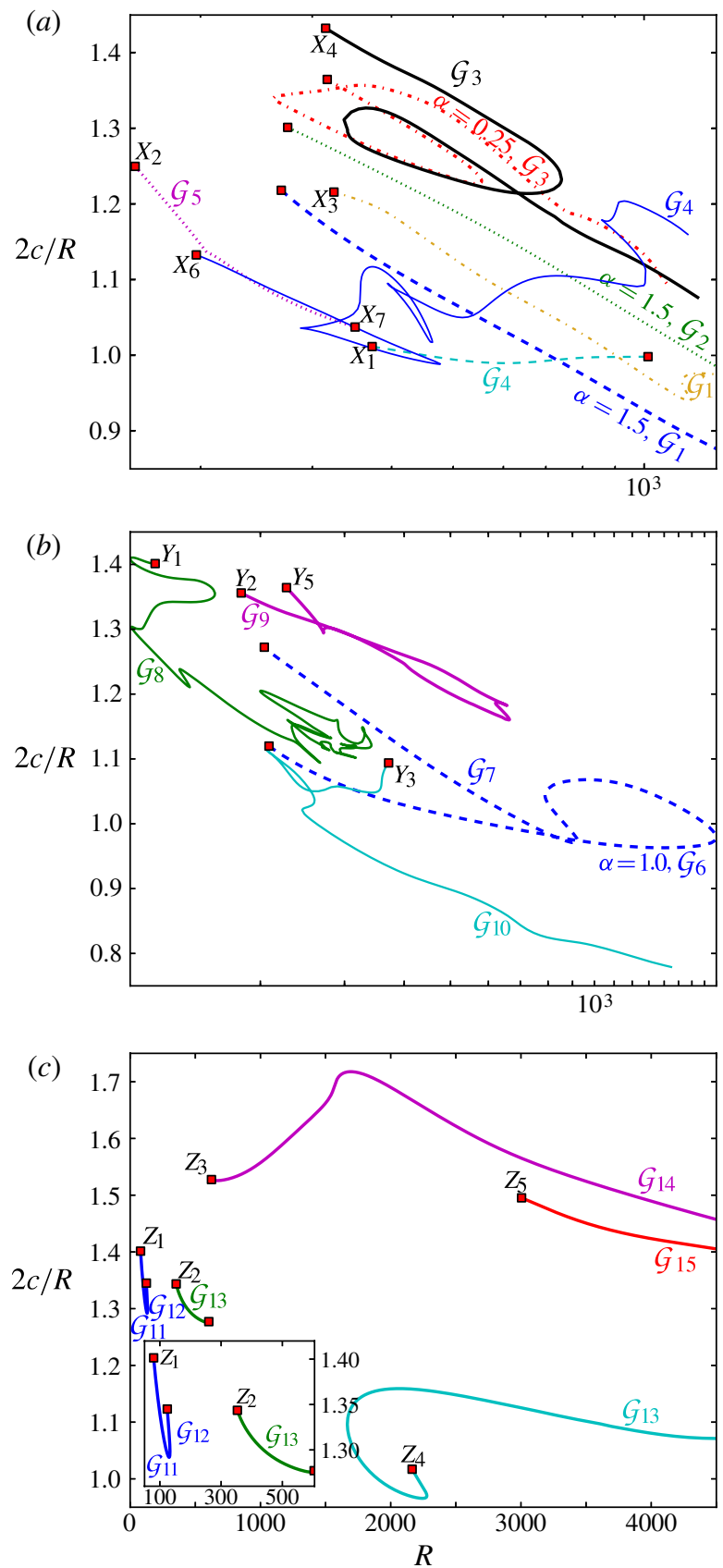

FIGURE 7. (Colour online) Wavespeeds scaled by $R / 2$ of the $(a)$ superharmonic, $(b)$ subharmonic tertiary flows and $(c)$ three-dimensional secondary flows bifurcating directly from the basic state. The wavespeeds of all flows can be observed to be $O(R / 2)$ for the range of $R$ for which solutions have been computed, with, however, $c /(R / 2)$ tending to decline with increasing $R$ in most cases. The largest wavespeeds are found for the secondary flows. The squares ( $\mathbf{G})$ again mark the location of the bifurcation points, while parameters and plot linestyles correspond to those used in $(a)$ figure 4, (b) figure 8 and (c) figure 11. 


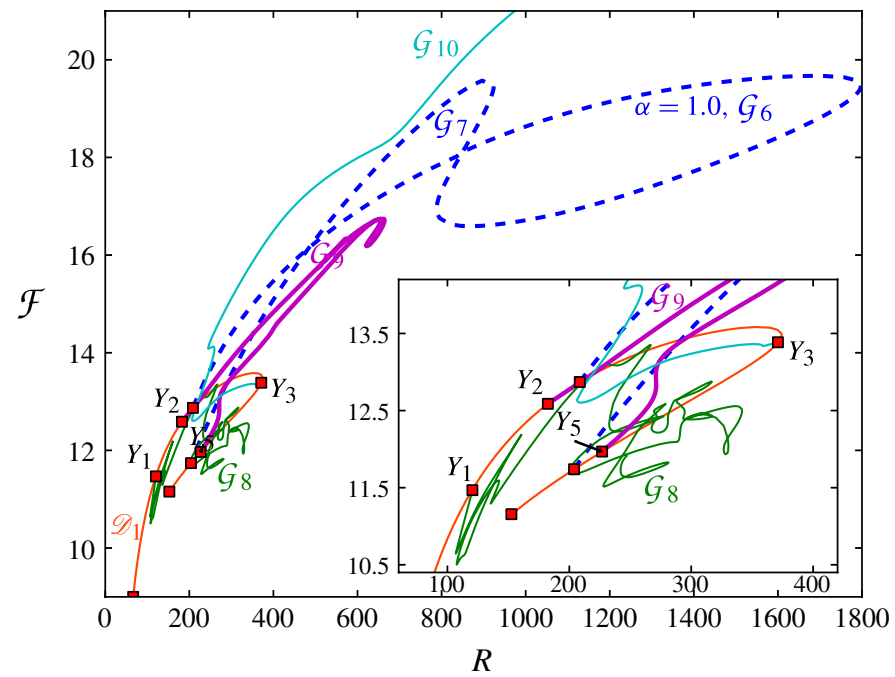

FIGURE 8. (Colour online) Bifurcation of the subharmonic three-dimensional travelling wave tertiary flows from the secondary flow $\left(\mathcal{D}_{1}\right)$. Friction factor $\mathcal{F}=R \lambda$ plotted against $R$ for fixed $\Omega=22.1325$. A rich solution structure also exists for the subharmonic solutions. All tertiary flow solutions have $\beta=1.25$, while $\alpha=0.2$ unless otherwise labelled. Bifurcation points are marked ( $\mathbf{\square})$, and the $\alpha=0.2$ solutions are additionally labelled according to figure 3. The following truncation levels $(L, M, N)$ were used: $\alpha=1.0,(33,9,20) ; Y_{1}$, $(25,19,16) ; Y_{2}$ and $Y_{5},(29,31,16) ; Y_{3},(29,15,18)$ and for the two-dimensional $\mathcal{D}_{1}$ solution $(15,0,7)$.

The solution bifurcating from point $X_{4}$, where the fourth least-stable eigenmode loses stability, also passes through two turning points before increasing with increasing $R$ as far as it has been tracked, to $R=1200$. With reference to figure 3 , it can be seen that the eigenmode that gives rise to the present solution upon its loss of stability at point $X_{4}$ corresponds to the least stable mode for $d=O\left(10^{-1}\right)$ on the upper branch of the secondary flow, and the flow structure (not shown) is accordingly qualitatively similar to the $\mathcal{G}_{3}$ flow shown in figure $6(b)$.

In figure 7 wavespeeds are plotted for the present superharmonic solutions and also for the subharmonic solutions shown in figure 8, and for the three-dimensional secondary flows shown in figure 11 . In each case the wavespeeds are approximately of magnitude $O(R / 2)$, in keeping with the empirical value of 'half the undisturbed centreline velocity' observed in the experiments of Alfredsson \& Persson (1989). However, the wavespeed of most of the flows declines against this measure with increasing $R$. The $\mathcal{G}_{4}$ flow that bifurcates from point $X_{1}$ and forms a bridge connecting two two-dimensional flows exhibits a wavespeed that is very close to $R / 2$ for every value of $R$ at which the flow exists. Figure 25 in the supplementary material provides a similar corresponding plot for all of the present solutions expressed in terms of a flux-based Reynolds number.

\subsection{Subharmonic tertiary flows}

Distinguishing on the basis of originating eigenmode and major solution branches (all subharmonic solutions share the same symmetry group $\mathscr{C}$ ), we identify five distinct subharmonic tertiary flows, $\mathcal{G}_{6}, \ldots, \mathcal{G}_{10}$. 

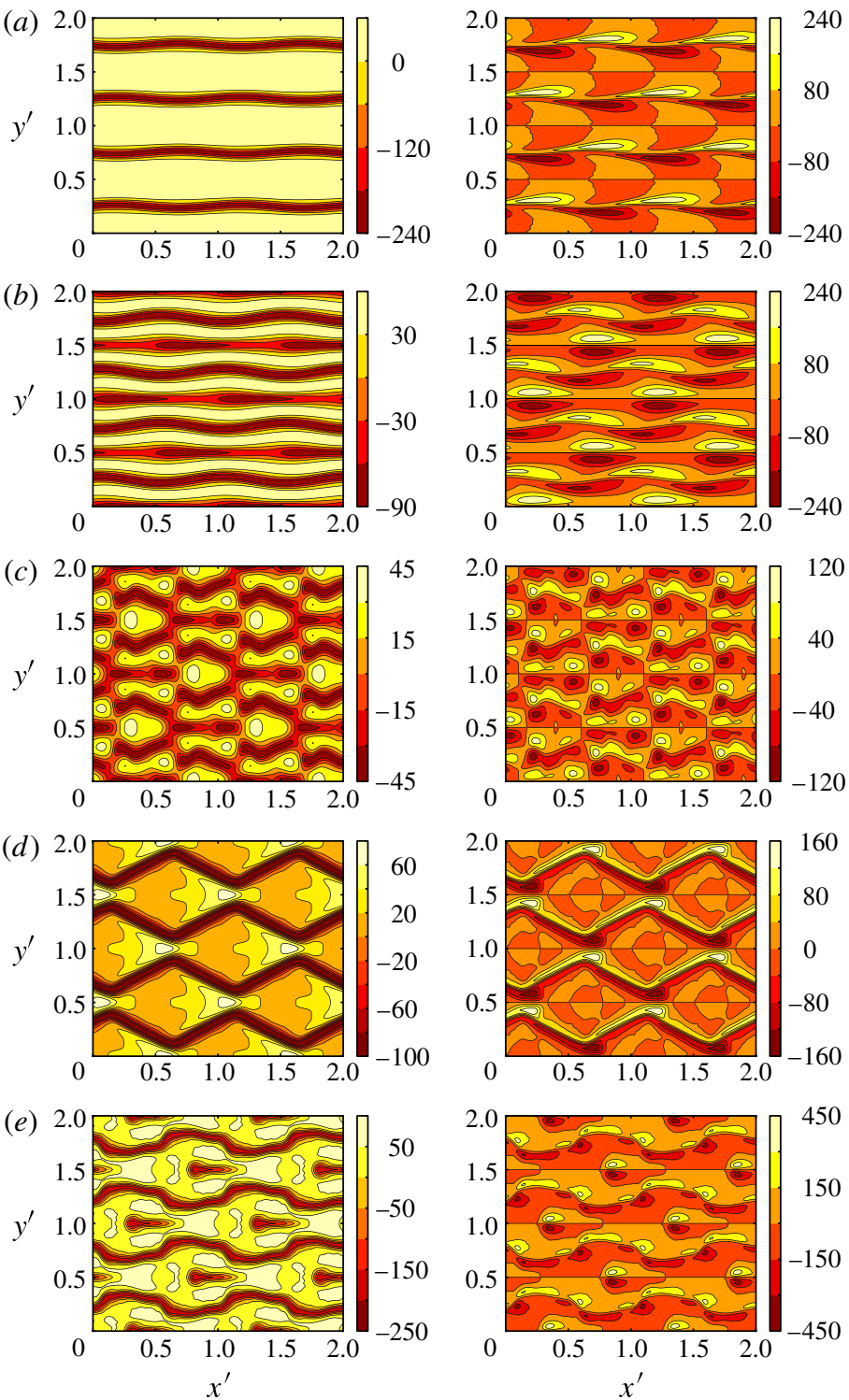

FIGURE 9. (Colour online) Visualization of (left) $u$ and (right) $\omega_{1}$ of subharmonic flows in a streamwise-spanwise plane. Although all flows satisfy the same symmetries, a variety of flow structures exists, still with, however, sinusoidal streaks flanked by staggered, and varicose streaks and low-speed pulses flanked by aligned vortices. When streaks merge more complicated patterns appear as in $(c)$. All flows are have parameters $(\beta, \Omega)=(1.25,22.1325)$ and are evaluated at $(L, M, N)=(29,8,16)$ unless otherwise stated. They bifurcate from: (a) $\left(\mathcal{G}_{6}\right)$ upper branch when $d=O(1) ;(R, \alpha)=(800,1.0)$ with $(L, M, N)=(33,9,20)$ at $z=0.691$; $(b)\left(\mathcal{G}_{7}\right)$ lower branch when $d=O(1),(R, \alpha)=(429,1.0)$ at $z=0.689$; (c) $\left(\mathcal{G}_{8}\right)$ upper branch when $d=O\left(10^{-1}\right)\left(Y_{1}\right.$ in figure 3$),(R, \alpha)=(200,0.2)$ with $(L, M, N)=(25,17,16)$ at $z=0.686 ;(d)\left(G_{9}\right)$ upper branch when $d=O\left(10^{-1}\right)\left(Y_{2}\right.$ in figure 3), $(R, \alpha)=(404,0.2)$ at $z=0.689 ;(e)\left(G_{10}\right)$ lower branch when $d=O\left(10^{-1}\right)\left(Y_{3}\right.$ in figure 3$),(R, \alpha)=(800,0.2)$ with $(L, M, N)=(29,13,18)$ at $z=0.612$. Two wavelengths are shown in $\left(x^{\prime}, y^{\prime}\right)=(1 / 2 \pi)(\alpha x, \beta y)$. 
$d=O(1)$

In figure 3 the real and imaginary parts of the six leading-order eigenmodes for the subharmonic disturbance for the representative value $d=1$ for varying $R$ in a domain that includes the turning point $R=374.1$ of the secondary flow are plotted. It can be seen that only a single eigenmode becomes unstable, with two bifurcation points; one on the upper and one on the lower branch of the secondary flow.

Two distinct subharmonic tertiary flows are identified by considering the solution arising in a bifurcation from the upper branch of the secondary flow when $(d, b)=(1, \grave{\beta} / 2=1.25)$. With reference to figure 8 , it can be seen that the solution moves in the direction of increasing $R$ before reaching a turning point and continuing along a lower branch that traverses two further turning points before eventually terminating on the lower branch of the secondary flow at the bifurcation point shown in figure 3 at $R=207$. (The corresponding plots for the flux-based Reynolds number $R_{f}$ are similar and are provided in figure 23 in the supplementary material.) The first two subharmonic tertiary flows to be identified in this section are given by the upper and lower branches of this solution. Figure $9(a)$ depicts the structure of this flow at $R=433$ on the upper branch, which we label $\mathcal{G}_{6}$. It can be seen that this flow is also distinguished by low-speed streamwise-orientated streaks in $u$. There are two streaks of equal strength per spanwise wavelength, which are sinusoidal in the streamwise direction and are flanked by staggered streamwise-orientated vortices. An animation of the velocity vectors in the spanwise-wall-normal plane for this flow is provided in supplementary movie 4 , where it can be seen that the flow in each half space $0 \leqslant y<\pi / \beta, \pi / \beta \leqslant y<2 \pi / \beta$ resembles the $d=O(1)$ upper-branch superharmonic flow in the full space $0 \leqslant y<2 \pi / \beta$.

The structure of the corresponding lower-branch flow, $\mathcal{G}_{7}$, at $R=429$ is shown in figure $9(b)$. This more complicated flow features a total of four low-speed streaks in $u$ per spanwise wavelength. Two of these streaks are sinusoidal (at $y^{\prime}=0.25$ and 0.75 , for example) while the other two are varicose (at $y^{\prime}=0$ and 0.5 , for example), with the two types appearing alternately in the spanwise direction. The sinusoidal streaks are flanked by staggered streamwise-orientated vortices, while the varicose streaks are flanked by aligned streamwise-orientated vortices. As can also be seen from supplementary movie 5, which shows an animation of the velocity vectors in the spanwise-wall-normal plane, the sinusoidal streaks are of equal magnitude while the two varicose streaks are of different magnitude and shape. The vortices associated with the varicose streaks exhibit almost no spanwise movement while those associated with the sinusoidal streaks exhibit the same spanwise rocking movement with the vortex on one side of the streak strongest when the other is weakest observed for the other sinusoidal streak flows described above. With further movement along the lower branch the varicose streaks separate into isolated low-speed pulses before vanishing completely when the flow moves to the upper branch, and the flow becomes the $\mathcal{G}_{6}$ flow described above.

$d=O\left(10^{-1}\right)$

In considering the case $d=O\left(10^{-1}\right)$, we consider the representative example $d=0.2$, for which the real and imaginary parts of the six leading-order eigenmodes for $R$ varying are plotted in figure 3. It can be seen that there are a total of six bifurcation points from the secondary flow, two $\left(Y_{1}, Y_{2}\right)$ on the upper and four $\left(Y_{3}, \ldots, Y_{6}\right)$ on the lower branch, for which we determine the values of $R$ to be given by $\left(R^{Y_{1}}, R^{Y_{2}}, R^{Y_{3}}, R^{Y_{4}}, R^{Y_{5}}, R^{Y_{6}}\right)=(121,182,372,369,227,154)$. For two of these neutral stability points, $Y_{4}$ and $Y_{6}$, we were unable to find bifurcating solutions. 
In the case of $Y_{4}$, the initial guesses that converged under the Newton-Raphson iteration, converged to the nearby $Y_{3}$ solution. (Furthermore, changing $d$ so that the $Y_{4}$ neutral stability point became more isolated from $Y_{3}$ resulted in initial guesses instead converging back to the two-dimensional secondary flow.) For the case of $Y_{6}$, this neutral stability point lies close to the point of the secondary flow's own bifurcation from a $\grave{\beta}=5$, two-dimensional solution branch (point $\mathrm{F}$ in figure 6 of Wall \& Nagata (2006)), and all of the initial guesses that converged, converged to this secondary flow.

The third subharmonic tertiary flow, $\mathcal{G}_{8}$, is given by the solution for the tertiary flow bifurcating from the upper branch of the secondary flow at $R=121$ (point $Y_{1}$ of figure 3), which is shown in figure 8 . The bifurcation curve first moves away from the bifurcation point in the direction of decreasing $R$, before then passing through a large number of turning points while remaining in the domain $100<R<350$ up to the point at which the tracking was abandoned. The flow structure near the bifurcation point (not shown) is somewhat similar to that shown in figure 9 for the $\mathcal{G}_{9}$ flow bifurcating from $Y_{2}$, but with the additional appearance of low-speed pulses in $u$ that appear once per streamwise wavelength near to the points where two neighbouring sinusoidal low-speed streaks in $u$ are closest. With further movement away from the bifurcation point the flow structure is as shown in figure 9, in which neighbouring sinusoidal streaks have joined at their closest points together with the low-speed pulses, thereby breaking the long streaks to form instead two-pronged fork shapes of low-speed $u$.

A fourth subharmonic tertiary flow is given by the solution that bifurcates from the secondary flow at point $Y_{2}$ of figure 3 , where the least-stable eigenmode that destabilized at point $Y_{1}$ restabilizes. With reference to figure 8 , it can be seen that the solution moves in the direction of increasing $R$ until a turning point is reached at around $R=660$. Thereafter the solution moves back in the direction of decreasing $R$, passing two further turning points before terminating on the lower branch of the secondary flow solution at $R=227$, which corresponds to point $Y_{5}$ of figure 3 . In particular, we identify the upper-branch flow, which is visualized in figure 9 , as the fourth subharmonic flow, $\mathcal{G}_{9}$. It can be seen that the flow features two sinusoidal low-speed streaks per spanwise wavelength of equal strength, which are accompanied by staggered vortices. In contrast to the flow bifurcating from point $Y_{1}$, no low-speed pulses are observed. Furthermore, while the flow is thus characterized by the same general flow features as the $\mathcal{G}_{6}$ upper-branch subharmonic flow for $d=O(1)$, the amplitude of the fluctuations of the low-speed streaks in the spanwise direction is an order of magnitude larger for the present flows. The flow on the lower branch (not shown) is qualitatively similar to the flow bifurcating from point $Y_{1}$ close to the bifurcation point (i.e. similar to figure $9 d$, but with the addition of a line of low-speed pulses between each pair of low-speed streaks that are flanked by aligned vortices). In fact, with reference to figure 3 , it may be noted that all three bifurcation points $Y_{1}, Y_{2}$ and $Y_{5}$ correspond to changes in stability of the same eigenmode: the eigenmode that becomes unstable at point $Y_{1}$ and then restabilizes at $Y_{2}$, again becomes unstable at point $Y_{5}$.

The fifth subharmonic tertiary flow, $\mathcal{G}_{10}$, is given by the flow bifurcating from point $Y_{3}$ of figure 3 (at $R=372$ ). It can be seen that the bifurcation curve first moves in the direction of decreasing $R$ until reaching a turning point at $R=206$, thereafter, after passing through two further turning points at $R=260$ and $R=245$, the bifurcation curve continues in the direction of increasing $R$ as far as it has been traced, up to $R=1400$, and produces the largest values of $\mathcal{F}$ found for the subharmonic flows for 
values of $R$ at which the solution exists. In figure 9 we visualize this flow at $R=261$, slightly beyond the third turning point. The flow features low-speed streaks in $u$ interspersed with lines of low-speed pulses in $u$, with the former flanked by staggered vortices and the latter flanked by aligned vortices. Although arising from a different eigenmode, the flow thus bears some similarity to the upper branch flow bifurcating from point $Y_{2}$ described above. However, for the present flow the streaks exhibit a somewhat crinkly appearance, and the extent of the spanwise undulation of the streaks is less than that of the $Y_{2}$ upper-branch flow (indeed the streaks do not merge, as was found for the flow bifurcating from $Y_{1}$ ). An animation of the velocity vectors in the spanwise-wall-normal plane for this flow provided in supplementary movie 6; four or six vortices are visible at any time depending on whether the snapshot is taken at a streamwise station without or with a low-speed pulse in $u$, respectively.

\subsection{Flows bifurcating from basic flow}

In analysing the stability of the basic flow, although the steady two-dimensional streamwise-invariant modes were found to have the largest growth rates, both Yang \& Kim (1991) and Wall \& Nagata (2006) found the growth rates of three-dimensional modes to be of comparable order. Furthermore, Yang \& Kim (1991) detected the presence of such three-dimensional modes with substantial amplitude in the early stages of the nonlinear transition presented in their simulations. For completeness, it is therefore of interest to find three-dimensional secondary flows that bifurcate directly from the basic state. Higher-order eigenmodes can also become unstable, and we here consider bifurcations from the three least stable eigenmodes that will be shown to yield a further five distinct three-dimensional flows, $\mathcal{G}_{11}, \ldots, \mathcal{G}_{15}$. All of the eigenmodes considered are of a single complex type with a non-zero imaginary part (see Wall \& Nagata (2006, figure 3(d)) for further details of the least-stable mode). We thus again anticipate a travelling wave solution, and a consideration of the symmetry of the bifurcating flow finds that these solutions are described by the same symmetry group, $\mathscr{C}$ in appendix A, as that of the subharmonic tertiary flows described above.

Considering the three primary spanwise disturbance wavenumbers $\beta=2.5,5.0$ and 7.5, we find it convenient to consider bifurcations of the least-stable eigenmode when $\beta=2.5$, the second least-stable eigenmode when $\beta=5.0$ and the third least-stable eigenmode when $\beta=7.5$. As shown in figure 10 , for the first two cases, selecting the primary streamwise disturbance wavenumber $\alpha=0.7$ and 0.35 , respectively, we find two bifurcation points $Z_{1}, Z_{2}$ and $Z_{3}, Z_{4}$, respectively, while a further potential bifurcation point, $Z_{5}$, is given by fixing $\alpha=0.1$ for the third least-stable eigenmode, where $\left(R_{Z_{1}}, R_{Z_{2}}, R_{Z_{3}}, R_{Z_{4}}, R_{Z_{5}}\right)=(79.3,352.8,623.0,2164.3,3004.0)$.

Two distinct flows bifurcating from the basic flow are given by the upper and lower branches of the flow arising in a bifurcation from point $Z_{1}$. It can be seen with reference to the bifurcation diagram plotted in figure 11 that the bifurcation curve moves in the direction of increasing $R$ until a turning point is reached, whereupon the solution moves in the direction of decreasing $R$ until terminating at a bifurcation from the upper branch of the two-dimensional flow with $\beta=5$. For reference, the corresponding plots for the flux-based Reynolds number $R_{f}$ are provided in figure 24 in the supplementary material. The structure of the upper branch flow, which we label $\mathcal{G}_{11}$, is shown in figures 12 and 13 , and can be further understood from the animation of velocity vectors in the spanwise-wall-normal plane provided in supplementary movie 7. It can be seen to feature a single layer of low-speed pulses, accompanied by aligned vorticity, with two lines of such pulses per spanwise wavelength. It can be seen that this flow features a somewhat simpler streamwise pattern than the tertiary 
(a)

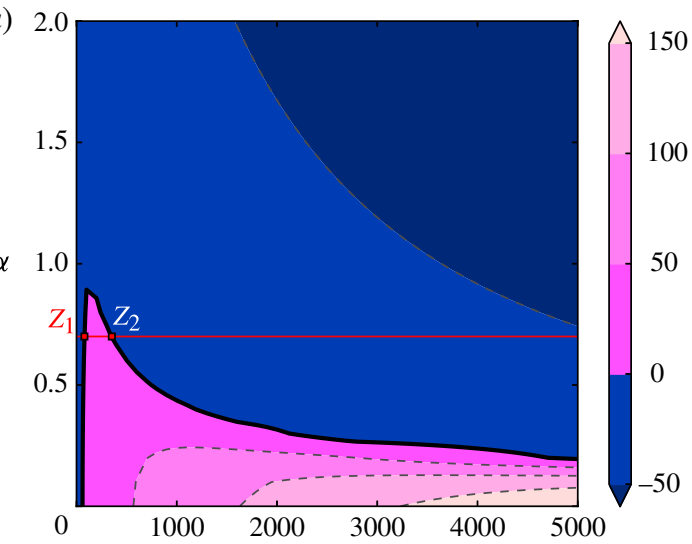

(b)

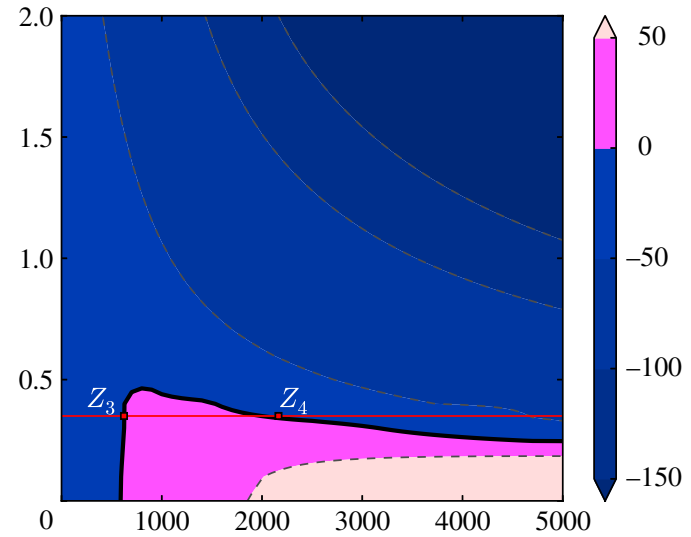

(c)

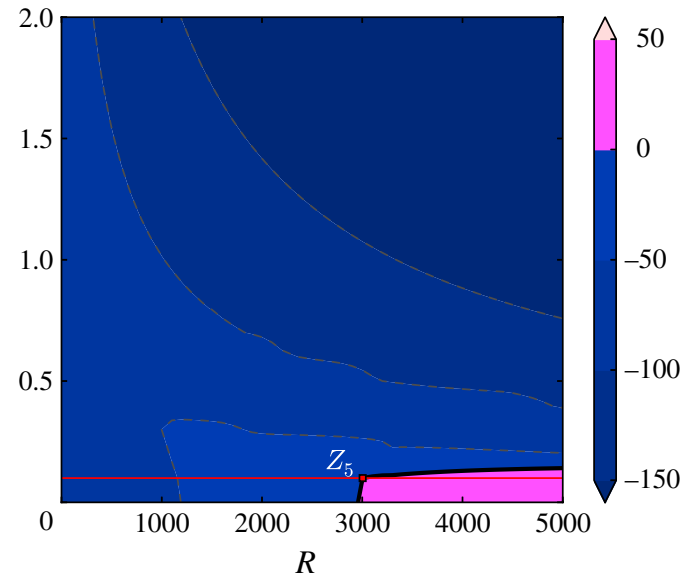

FIGURE 10. (Colour online) Bifurcation points for three-dimensional flows bifurcating directly from the basic state. Contours of the real part of the $(a)$ least-stable eigenvalue mode when $(\beta, \Omega)=(2.5,22.1325),(b)$ second least-stable eigenvalue mode when $(\beta, \Omega)=$ $(5.0,22.1325)$ and $(c)$ third least-stable eigenvalue mode when $(\beta, \Omega)=(7.5,22.1325)$, with all numerical calculations performed using the numerical method described by Wall \& Nagata (2006, §3) with $L=45$. 


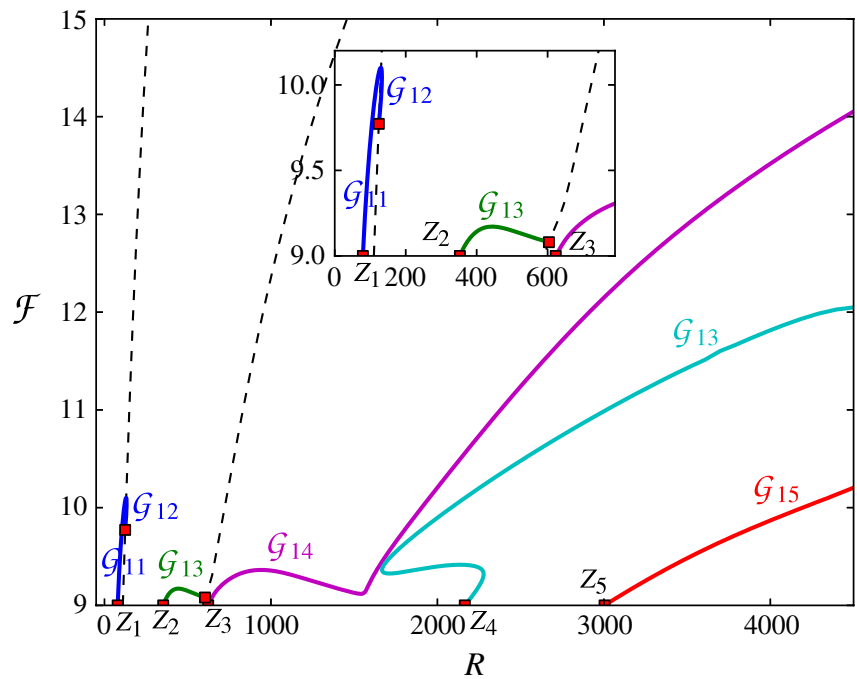

FIGURE 11. (Colour online) Friction factor $\mathcal{F}=R \lambda$ against $R$ for the three-dimensional travelling wave solutions bifurcating direct from the basic state. Flows are shown bifurcating from: $\left(Z_{1}\right)$ flow types $\mathcal{G}_{11}$ (upper branch) and $\mathcal{G}_{12}$ (lower branch), obtained for $(\alpha, \beta)=$ $(0.7,2.5)$ with $(L, M, N)=(29,13,12) ;\left(Z_{2}\right)$ flow type $\mathcal{G}_{13}$, obtained for $(\alpha, \beta)=(0.7,2.5)$ with $(L, M, N)=(39,8,12) ;\left(Z_{3}\right)$ flow type $\mathcal{G}_{14}$, obtained for $(\alpha, \beta)=(0.35,5.0)$ with $(L, M, N)=(71,14,10) ;\left(Z_{4}\right)$ flow type $\mathcal{G}_{13}$, obtained for $(\alpha, \beta)=(0.35,5.0)$ with $(L, M, N)=(61,9,8)$; and $\left(Z_{5}\right)$ flow type $\mathcal{G}_{15}$, obtained for $(\alpha, \beta)=(0.1,7.5)$ with $(L, M, N)=(89,14,9)$. Also shown (- $)$ is the two-dimensional solution bifurcating from the basic flow for $(\alpha, \beta)=(0,5.0)$ with $(L, M, N)=(45,0,14)$. All flows have $\Omega=22.1325$.

flows. This is generally true of the three-dimensional secondary flows, which have undergone one fewer symmetry-breaking bifurcation. The lower branch flow, $\mathcal{G}_{12}$, is instead described by a single layer of varicose low-speed streaks again accompanied by aligned vortices, with two streaks per spanwise wavelength.

The flow arising from bifurcation point $Z_{2}$ moves in the direction of increasing $R$ before terminating at a bifurcation from the lower branch of the same two-dimensional flow with $\beta=5$ that provides the bifurcation point for the $\mathcal{G}_{12}$ flow. The lower branch of this two-dimensional flow bifurcates due to the change in stability of a different eigenmode (the second least stable) to the one that provides the upper branch (the least-stable eigenmode), and features a two-layer structure rather than the single-layer structure of the upper branch. (This structure can be seen for different parameter values in figure $20 d$ in comparison with the single-layer structure in figure 20a.) The present bifurcating three-dimensional flow, which we label $\mathcal{G}_{13}$, inherits this two-layer structure as is shown in figures 12 and 13, including flow structures that can straddle both layers. The upper layer features high-speed pulses that are slightly stronger than sinusoidal low-speed streaks, with the former flanked by aligned, and the latter by staggered vortices. In the lower layer the situation is reversed, with low-speed pulses slightly stronger than high-speed sinusoidal streaks. The two-layer structure can also be seen in the animation of velocity vectors in the spanwise-wall-normal plane provided in supplementary movie 8 . 

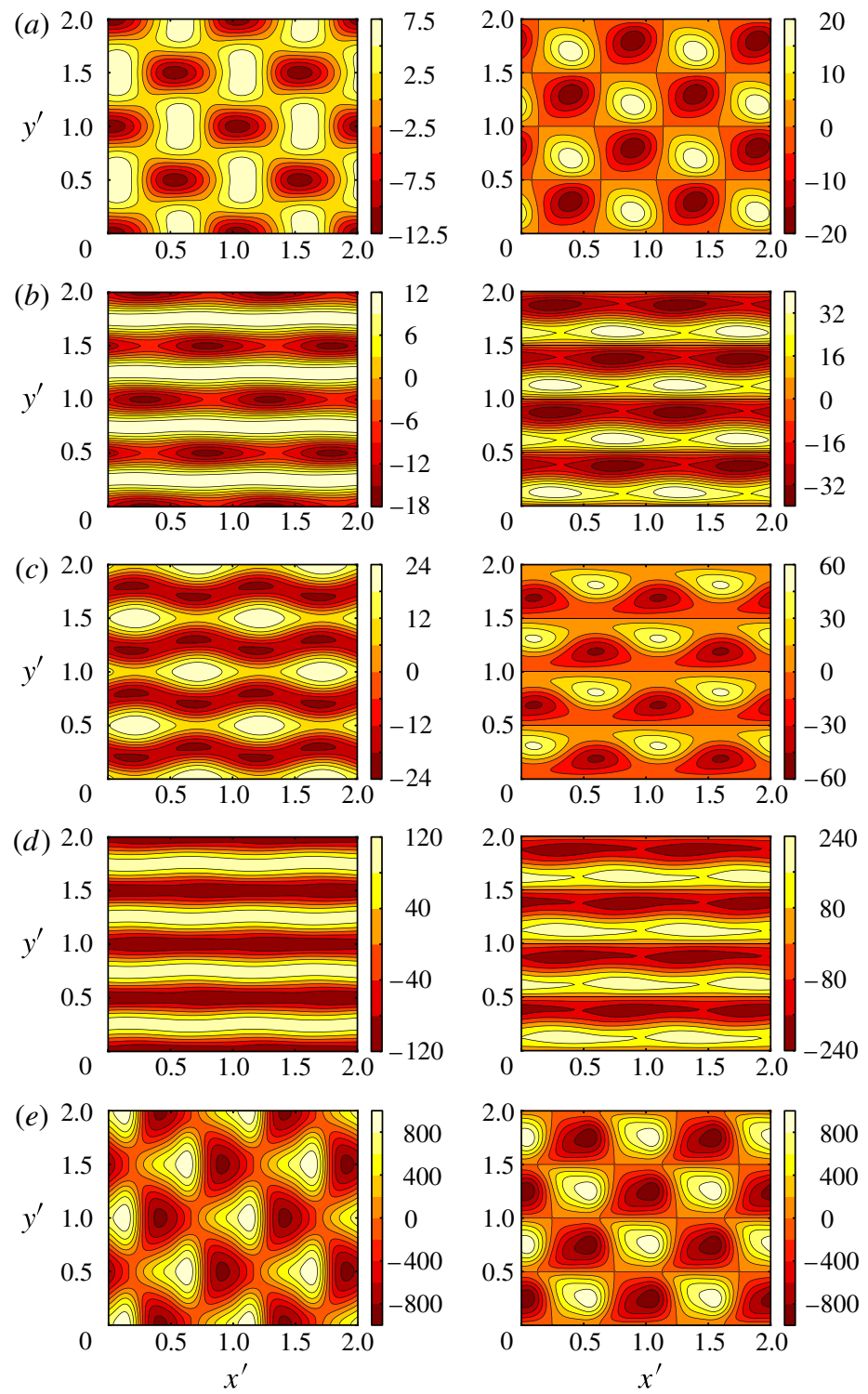

FIGURE 12. (Colour online) Visualization of (left) $u$ and (right) $\omega_{1}$ in a streamwise-spanwise plane of three-dimensional secondary flows bifurcating directly from the basic state. The streamwise structure of these flows is generally simpler than that of the tertiary flows, having undergone one fewer symmetry-breaking bifurcation than the latter: $(a)$ upper branch of flow (type $\left.\mathcal{G}_{11}\right)$ bifurcating from point $Z_{1}$, shown at $z=0.766$ for $(R, \alpha, \beta)=(100,0.7,2.5)$ with $(L, M, N)=(25,12,10)$; $(b)$ lower branch of flow (type $\left.\mathcal{G}_{12}\right)$ bifurcating from point $Z_{1}$, shown at $z=0.612$ for $(R, \alpha, \beta)=(126,0.7,2.5)$ with $(L, M, N)=(29,13,12)$; (c) flow (type $\mathcal{G}_{13}$ ) bifurcating from point $Z_{2}$, shown at $z=0.771$ (for $(R, \alpha, \beta)=(548,0.7,2.5$ ) with $(L, M, N)=(39,8,12)$; $(d)$ flow (type $\left.\mathcal{G}_{14}\right)$ bifurcating from point $Z_{3}$, shown at $z=0.375$ for $(R, \alpha, \beta)=(2003,0.35,5.0)$ with $(L, M, N)=(43,8,8)$; (e) flow (type $\left.\mathcal{G}_{15}\right)$ bifurcating from point $Z_{5}$, shown at $z=0.568$ for $(R, \alpha, \beta)=(11199,0.1,7.5)$ with $(L, M, N)=(89,13,9)$. All flows for $\Omega=22.1325$, and two wavelengths are shown in $\left(x^{\prime}, y^{\prime}\right)=(1 / 2 \pi)(\alpha x, \beta y)$. 
(a)

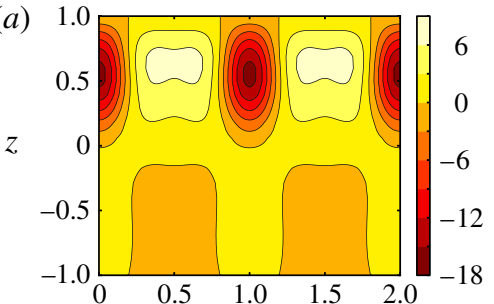

(b)

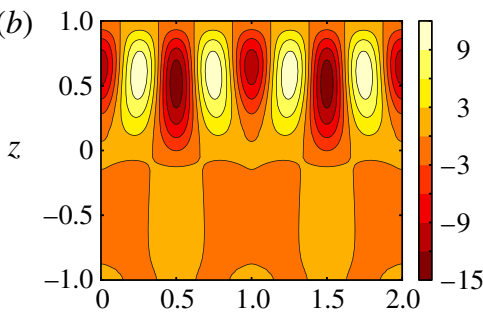

(c)

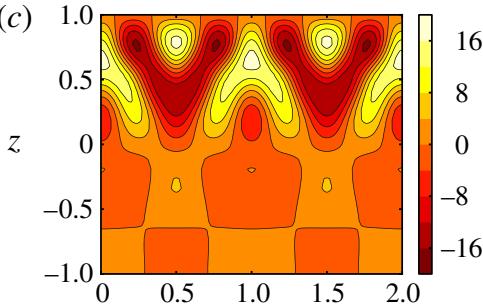

$(d)$

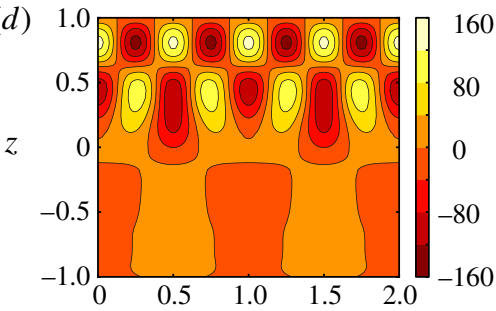

(e)

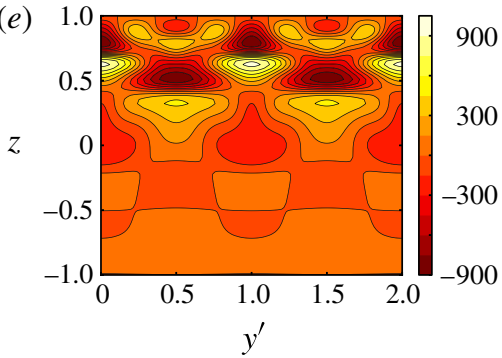

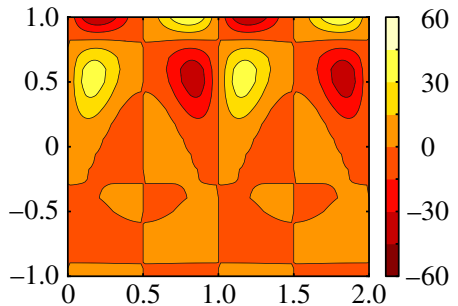
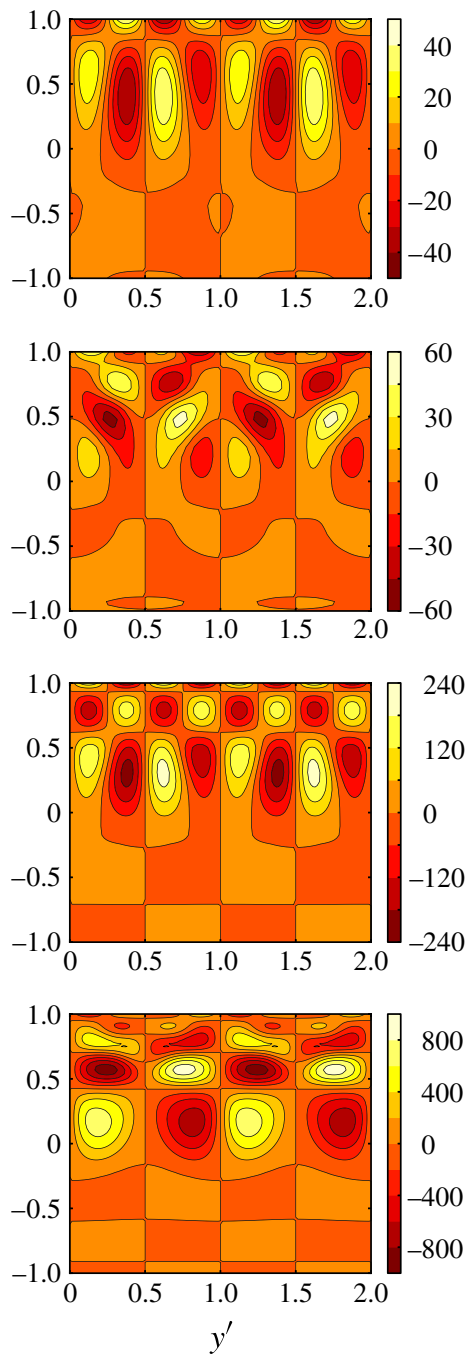

FIGURE 13. (Colour online) Visualization of (left) $u$ and (right) $\omega_{1}$ of three-dimensional flows bifurcating directly from the basic state in a spanwise-wall-normal plane $(x=0)$. As well as single-layer structures $(a, b)$, in contrast to the tertiary flows, double $(c, d)$, triple and quadruple $(e)$ layer structures can be found for these secondary flows. Two wavelengths are shown in $y^{\prime}=(1 / 2 \pi)(\beta y)$, and all parameters are as in figure 12 . 
The flow bifurcating from the point $Z_{3}$, where the second least-stable eigenmode loses stability, moves in the direction of increasing $R$ as far as it has been tracked, up to $R=5500$. This flow produces larger values of $\mathcal{F}$ than those found for the flows bifurcating from points $Z_{4}$ and $Z_{5}$ at every value of $R$ at which the solution exists. This flow also features a two-layer structure. Initially both layers are given by lines of low-speed pulses, with two such lines per spanwise wavelength. With further movement along the solution path, however, the layer closest to the $z=1$ wall features quasi two-dimensional low- and high-speed streaks of almost equal magnitude, while the second layer features varicose low-speed streaks slightly stronger than sinusoidal high-speed streaks and accompanied by aligned vortices. The streamwise invariance of the layer of vortices adjacent to the $z=1$ wall for this flow, which we label $\mathcal{G}_{14}$, can also clearly be seen in the animation of velocity vectors in the spanwise-wall-normal plane provided in supplementary movie 9.

The flow bifurcating from point $Z_{4}$ first moves in the direction of increasing $R$ until reaching a turning point at $R=2280$ with the flow moving in the direction of decreasing $R$ before passing through a second turning point at $R=1670$ and then continues in the direction of increasing $R$ as far as the solution has been tracked, up to $R=4000$. Further analysis reveals that the eigenmode that changes stability at $Z_{1}$ and $Z_{2}$ is the same mode that changes stability at $Z_{4}$, and the flow pattern (not shown) is qualitatively similar to that of flow $\mathcal{G}_{13}$ described above, and so is not recognized as distinct in the present study. It may be noted, however, that the roles of high and low speed are reversed in the present flow in comparison with flow $\mathcal{G}_{13}$.

The final flow considered in this section, which we label $\mathcal{G}_{15}$, bifurcates from point $Z_{5}$ and moves in the direction of increasing $R$ as far as it has been tracked, up to $R=11000$. The structure of this flow is initially triple-layered near the bifurcation from the basic flow, but has become quadruple-layered by the time it reaches $R=10000$, as is shown in the visualization in figures 12 and 13. The closest (and weakest) of these four layers to the channel wall $z=1$ features varicose low-speed streaks flanked by aligned vortices, the second and fourth layers feature low-speed pulses, while the third layer (shown in figure 12) features high-speed pulses slightly stronger than low-speed pulses that are also present. The quadruple-layer structure of this flow can also be seen in the animation of velocity vectors in the spanwise-wallnormal plane provided in supplementary movie 10 .

A plot of the wavespeeds for these secondary flows is provided in figure 7 . It can be seen that, similar to the tertiary flows, the present wavespeeds are also generally of size $O(R / 2)$, with $c /(R / 2)$ declining as $R$ increases for flows whose bifurcation curves do not terminate.

\section{Mean absolute vorticity}

Absolute vorticity denotes the vorticity of the flow expressed in an inertial frame. For turbulent flows, a frequently observed but as yet poorly understood phenomenon is the appearance of a region in the centre of the channel in which the magnitude of mean absolute vorticity (i.e. the spanwise-streamwise-averaged absolute vorticity) is small, often referred to as zero mean absolute vorticity (see the DNS study of Kristoffersen \& Andersson (1993), for example). It is of interest whether such a phenomenon is also manifested in the present transitional flows.

For the present case only the spanwise component of mean absolute vorticity is non-zero, and it is convenient to consider the dimensionless form of this component 
expressed for the present configuration that is given by

$$
\omega_{A}=-\frac{1}{2 R}\left(\frac{\mathrm{d} \check{U}}{\mathrm{~d} z}+\frac{\mathrm{d} u_{0}}{\mathrm{~d} z}+\Omega\right) .
$$

In figure 14 we plot $\omega_{A}$ for a number of the present three-dimensional travelling wave solutions at $R=800$ (with the exception of the $G_{14}$ flow, for which $R=800$ is too close to the bifurcation point $Z_{3}$ for the $\omega_{A}$ profile to have changed significantly from the linear laminar profile), along with the corresponding mean flow profiles. It is clear that for most of the flows there is a region centred in the $0 \leqslant z \leqslant 1$ half of the channel in which $\omega_{A}$ assumes a flattened profile, and is generally small. However, it is also clear that for some of these solutions this behaviour is only poorly manifested (e.g. the $\mathcal{G}_{3}$ flows), while for other solutions it cannot be claimed that such a region exists (e.g. the $\mathcal{G}_{4}$ flow bifurcating from $X_{6}$ ). In contrast, with reference to figure 15 , it can be seen that this phenomenon is more clearly manifested for the streamwise-independent two-dimensional flows that originate in bifurcations from the basic state. However, it is also noted that while the values of $\left|\omega_{A}\right|$ are generally small, it is not the case that the values of $\omega_{A}$ are approaching zero in the flattened regions. For example, for the case of $\mathcal{G}_{4}$ bifurcating from $X_{1}$ it can be seen that the value of $\mathrm{d} U / \mathrm{d} z>0$. Furthermore, as in the case of the lower-branch two-dimensional flow for $\Omega=27.1$ shown in figure 15 for example, we can also observe flows for which two such regions of flattened $\omega_{A}$ exist, with $\left|\omega_{A}\right|$ of $O(1)$ in the flattened region closest to the $z=1$ wall. A similar phenomenon can be observed for the three-dimensional $G_{14}$ flow shown in figure 14, in which the region of flattened $\omega_{A}$ is manifested much more clearly in the quasi-two-dimensional layer closest to the $z=1$ wall than in the second layer, in which the flow structures vary more strongly in the streamwise direction. Visualizations of the flows whose absolute vorticity is considered in figure 14 can be found in figures 5, 6, 9, 12 and 13, with the remaining flows visualized in figure 16. It is clear that the three-dimensional flows that do not exhibit the present flattening of the $\omega_{A}$ profile phenomenon either show a large streamwise dependence, with low-speed streaks and their accompanying vortex structures fluctuating across a full spanwise wavelength (or half a spanwise wavelength in the case of symmetry group $\mathscr{C}$ ), for example the $\mathcal{G}_{3}$ flows, or the streak structure has been lost entirely (e.g. the $\mathcal{G}_{4}$ flow bifurcating from $X_{6}$ ).

Against the background of these results, we thus have that for the present transitional flows there is a phenomenon described by a flattening of the profile of $\omega_{A}$ in region(s) of the channel, with $\left|\omega_{A}\right|$ typically small, but not necessarily approaching zero, if the region lies close to the centre of the channel, and of $O(1)$ otherwise. We propose that this phenomenon can be explained by a mechanism given by the redistribution of vorticity due to two-dimensional streamwise-independent roll cells, and is thus essentially the well-known lift-up mechanism occurring in a rotating frame. The mechanism is schematically illustrated in figure 17; the roll cells advect fluid of high $\omega_{A}$ near the $z=1$ channel wall towards the middle of the channel where $\omega_{A}$ is lower, thus raising the vorticity at the bottom of the vortex layer, while lowering it (by advecting fluid from the centre of the channel) near $z=1$. Thus, the profile of $\omega_{A}(z)$ becomes flattened in the region(s) where the streamwise vortices exist.

A simple two-dimensional model can further illustrate this result. We prescribe the simple two-dimensional roll-cell vortex structure given by

$$
\check{w}=W(z) \cos (\beta y),
$$



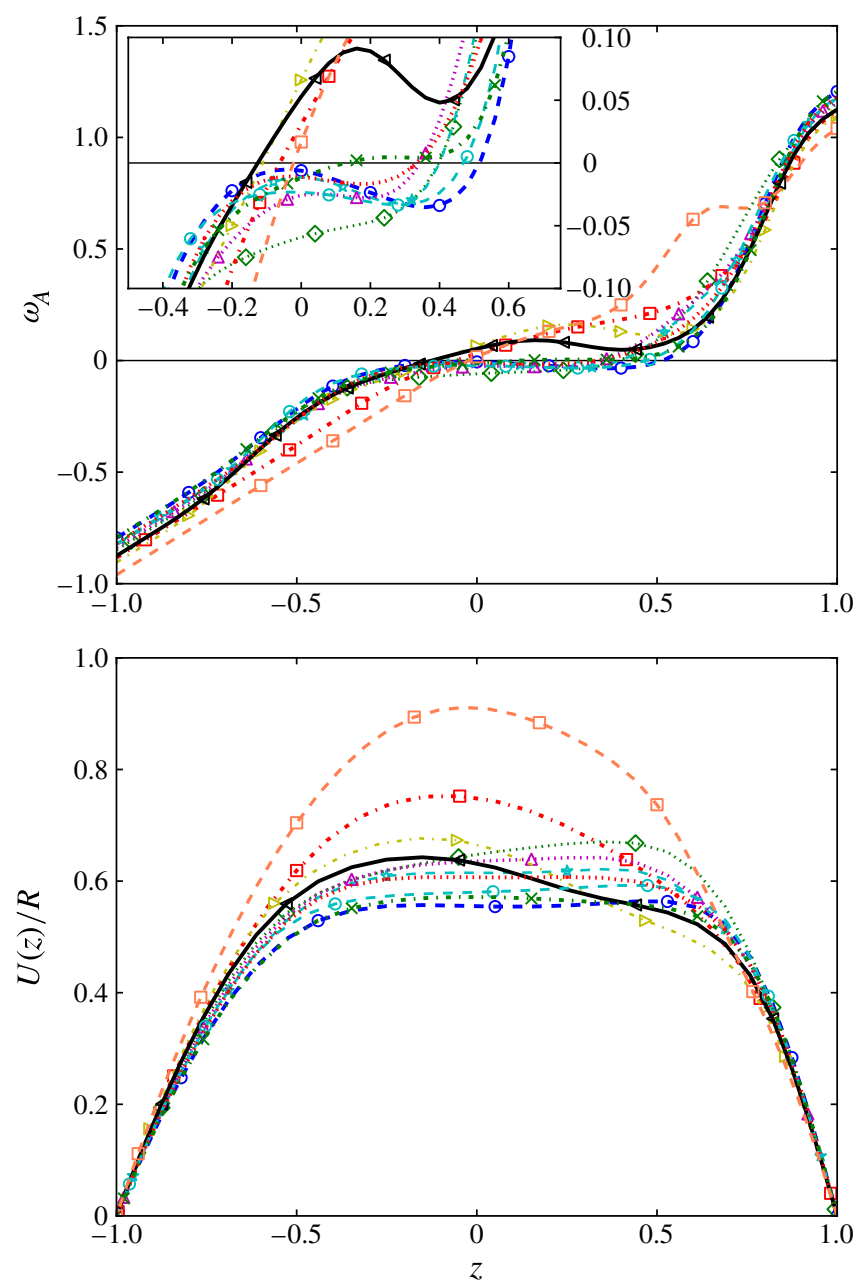

FIGURE 14. (Colour online) Mean absolute vorticity, $\omega_{A}$, and mean flow, $U(z)$, for a selection of the present three-dimensional flows: most flows feature a region in the middle of the channel centred in $0 \leqslant z \leqslant 1$ in which the absolute vorticity profile is flattened. However, for some flows (e.g. $\mathcal{G}_{4}$ from $\left.X_{1}\right) \mathrm{d} U / \mathrm{d} z>0$ in this region, in contrast to the phenomenon observed in fully turbulent flows in which $\mathrm{d} U / \mathrm{d} z \approx-\Omega<0$, so that $\omega_{A}$ approaches zero. All flows have $\Omega=22.1325$, and $R=800$ unless otherwise stated. All superharmonic flows are computed for $\beta=2.5$, and all subharmonic flows for $\beta=1.25$.

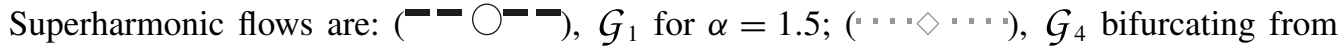
$X_{1}$ for $\alpha=0.4$; ("- $\left.\square \cdot-\right), \mathcal{G}_{4}$ bifurcating from $X_{6}$ for $\alpha=0.4,(L, M, N)=(31,9,9)$; $\left(--x^{\prime}--\right), \mathcal{G}_{2}$ from lower branch with $\alpha=1.5$; (" " $\triangle \triangle^{\prime \prime \prime), ~} \mathcal{G}_{2}$ bifurcating from $X_{3}$ for $\alpha=0.4 ;\left(^{--}\right), \mathcal{G}_{3}$ bifurcating from upper branch with $\alpha=0.25,(L, M, N)=$ $(27,21,8)$; ( $\triangleleft), \mathcal{G}_{3}$ bifurcating from $X_{4}$ for $\alpha=0.4,(L, M, N)=(29,14,9)$. Truncation level $(L, M, N)=(29,8,8)$ unless otherwise stated. Subharmonic flows are: $\left({ }^{-} \bigcirc^{\prime}\right), \mathcal{G}_{6}$ upper branch for $\alpha=1,(L, M, N)=(33,9,20) ;(\ldots \ldots), \mathcal{G}_{7}$ from lower branch for $\alpha=1,(L, M, N)=(29,8,16)$; $(--\times-=), \mathcal{G}_{10}$ bifurcating from $Y_{3}$ for $\alpha=0.2,(L, M, N)=(29,13,18)$. Three-dimensional secondary flow: $(--\square--), \mathcal{G}_{14}$ for $(R, \alpha, \beta)=(2003,0.35,5.0),(L, M, N)=(43,8,8)$. 


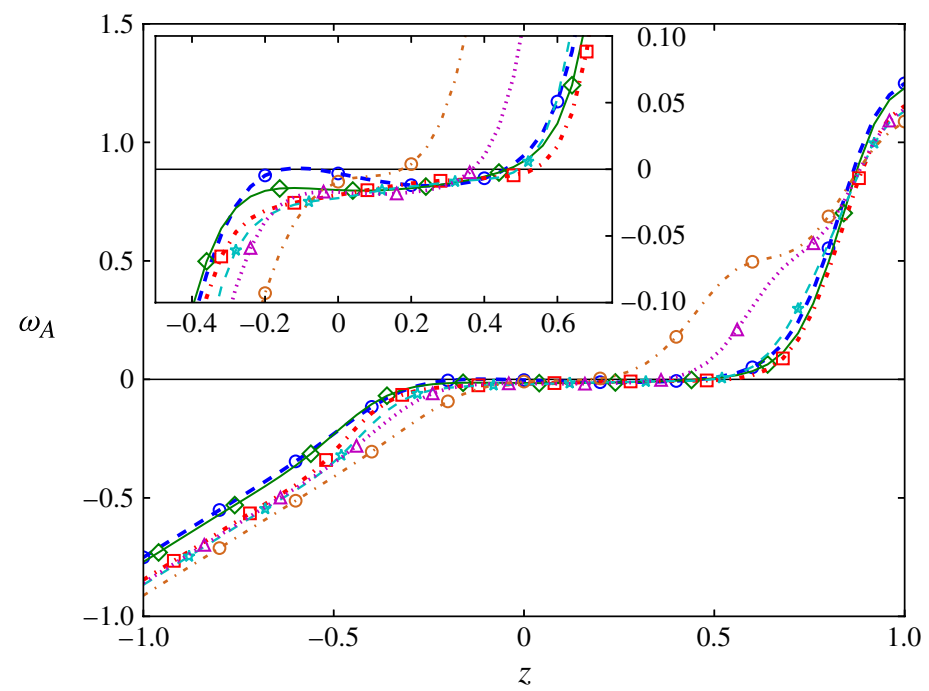

FIGURE 15. (Colour online) Mean absolute vorticity for two-dimensional flows bifurcating from the basic state: all flows exhibit a region in the middle of the channel centred in $0 \leqslant z \leqslant 1$ in which the absolute vorticity profile is flattened, while a second region of flattened $\omega_{A}$ can also be observed closer to the $z=1$ wall for some flows. Flows obtained for upper and lower branches of solution with $(R, \alpha, \beta)=(800,0,5),(L, M, N)=(27,0,11)$ and different values of $\Omega$ : (-ー $\bigcirc-$ ) $\Omega=27.2$ (upper); ( $\square \diamond-$ ) $\Omega=50.1$ (upper);

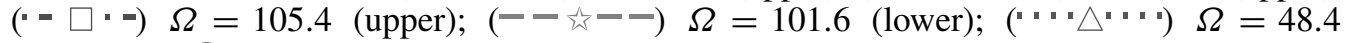
(lower); ( $\left.{ }^{-} \bigcirc{ }^{\prime \prime}\right) \Omega=27.1$ (lower).

and seek to solve the governing equations (2.5), (2.6) for

$$
\begin{aligned}
& u=u_{0}+\hat{u}=u_{0}+\check{U}+\check{u}=u_{0}+\check{U}+\check{u_{1}}(z) \cos (\beta y)+\check{u_{2}}(z) \cos (2 \beta y)+\cdots \\
& p=p_{0}+\hat{p}=p_{0}+\check{P}(z)+\check{p}(y, z)
\end{aligned}
$$

where $\check{U}, \breve{P}$ are the spanwise averages of $\hat{u}, \hat{p}$, respectively. The form for $W(z)$ is given by the eigenfunction of the mode whose change in stability yields bifurcation points for the two-dimensional finite-amplitude secondary flows, for which a series solution in the form

$$
W(\zeta)=\sum_{m=1}^{\infty} c_{m} w_{m}(\zeta)
$$

where

$$
\begin{aligned}
w_{m}= & \frac{-1}{\left(m^{2} \pi^{2}+a^{2}\right)^{3}}\left(A_{1}^{(m)} \cosh a \zeta+A_{2}^{(m)} \zeta \cosh a \zeta+A_{3}^{(m)} \zeta^{2} \cosh a \zeta+B_{1}^{(m)} \sinh a \zeta\right. \\
& \left.+B_{2}^{(m)} \zeta \sinh a \zeta+B_{3}^{(m)} \zeta^{2} \sinh a \zeta+(1+\chi \zeta) \sin m \pi \zeta+\frac{6 \chi m \pi}{m^{2} \pi^{2}+a^{2}} \cos m \pi \zeta\right)
\end{aligned}
$$



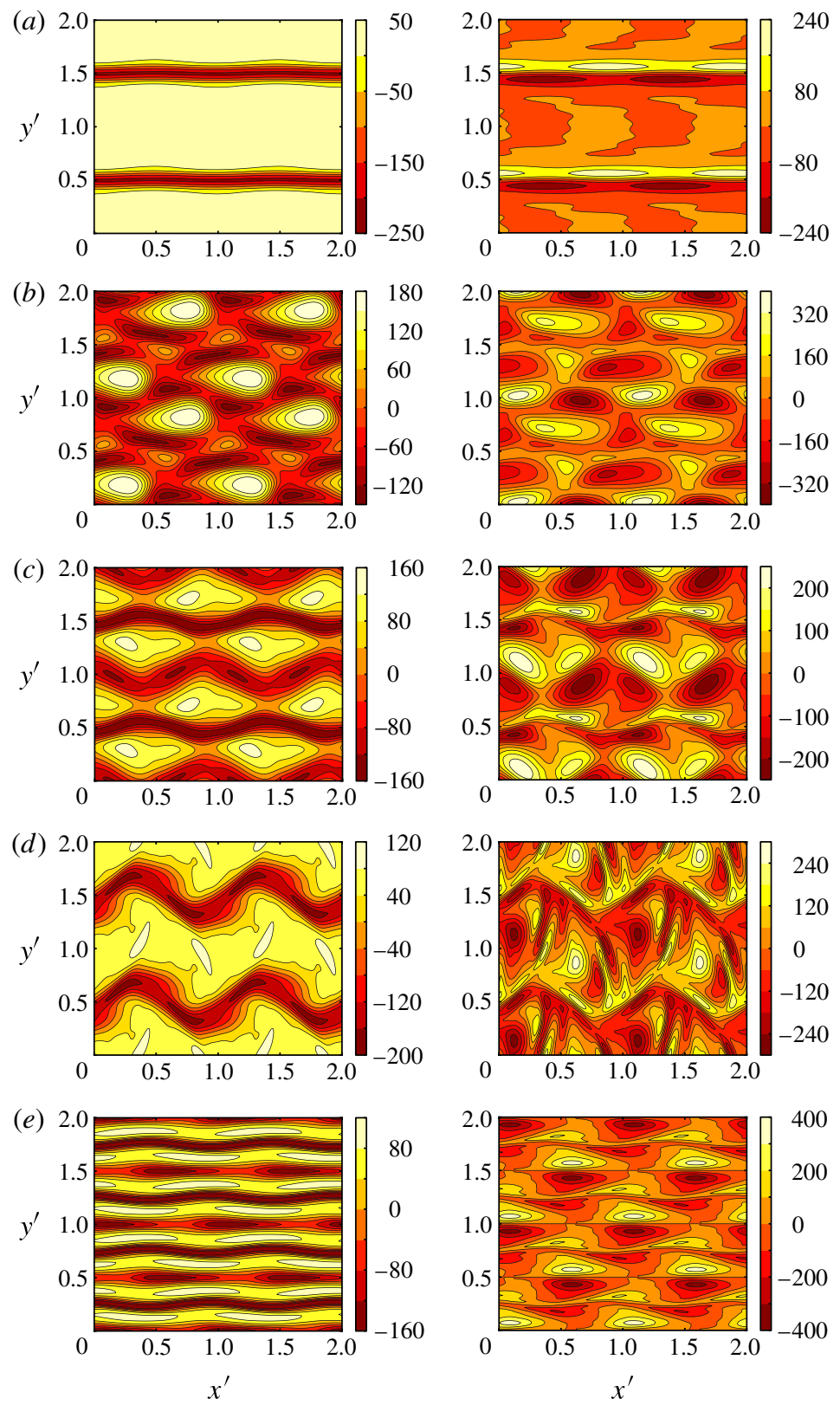

FIGURE 16. (Colour online) Visualization of (left) $u$ and (right) $\omega_{1}$ of flows in figure 14 not shown elsewhere. Flows that have lost their long streamwise structures, such as $(b)$, do not manifest the flattening of the $\omega_{A}(z)$ profile as clearly as other flows. Superharmonic flows $\left((L, M, N)=(29,8,8)\right.$ unless otherwise stated): (a) $\mathcal{G}_{4}$ bifurcating from $X_{1}$ for $\alpha=0.4$, at $z=0.612$; (b) $\mathcal{G}_{4}$ bifurcating from $X_{6}$ for $\alpha=0.4,(L, M, N)=(31,9,9)$ at $z=0.724 ;(c) \mathcal{G}_{2}$ bifurcating from $X_{3}$ for $\alpha=0.4$, at $z=0.759 ;(d) \mathcal{G}_{3}$ bifurcating from $X_{4}$ for $\alpha=0.4,(L, M, N)=(29,14,9)$ at $z=0.759$. Subharmonic flow: (e) $\mathcal{G}_{7}$ from lower branch for $\alpha=1,(L, M, N)=(29,8,16)$ at $z=0.759$. Two wavelengths are shown in $\left(x^{\prime}, y^{\prime}\right)=(1 / 2 \pi)(\alpha x, \beta y)$. 


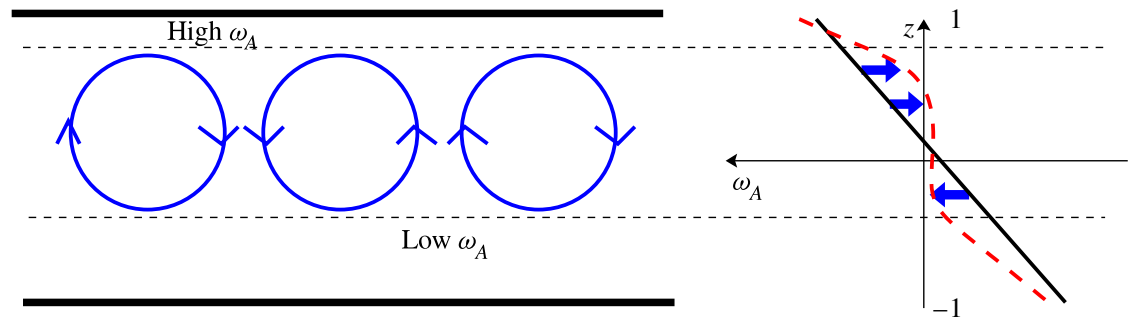

FIGURE 17. (Colour online) Schematic illustration of flattening of the absolute vorticity profile caused by mixing across a layer of streamwise roll cells. Fluid with high $\omega_{A}$ near the $z=1$ wall is advected towards the centre of the channel, while fluid of low $\omega_{A}$ is advected towards the $z=1$ wall. The thick black line in the right-hand plot shows the laminar profile, while the dashed line (shown in red online) shows the resultant flattened profile of $\omega_{A}$ observed in the present two-dimensional transitionary flows and in three-dimensional transitional flows that feature such streamwise vortex structures. If further layers of roll cells are present the mechanism operates across each layer.

can be derived in which $a=2 \beta, \chi=-4 R /(\Omega+2 R)$ and $\zeta=(z+1) / 2$ (see appendix B for further details). Substituting into (2.6) for $\breve{w}$ from expression (6.2) we derive

$$
\check{v}=-\frac{1}{\beta} W^{\prime}(z) \sin \beta y,
$$

and then, from the $y$ component of (2.5), we derive

$$
\check{p}=\frac{1}{4 \beta^{2}}\left[4\left(W^{\prime \prime \prime}-\beta^{2} W^{\prime}\right) \cos \beta y+\left(W^{\prime 2}-W W^{\prime \prime}\right) \cos 2 \beta y\right],
$$

while the $z$ component of the same equation yields

$$
\begin{aligned}
& W W^{\prime}+\left(\beta^{2} W-W^{\prime \prime}\right) \cos \beta y+\frac{1}{4 \beta^{2}}\left[4\left(W^{(4)}-\beta^{2} W^{\prime \prime}\right) \cos \beta y\right. \\
& \left.+\left(W^{\prime} W^{\prime \prime}-W W^{\prime \prime \prime}\right) \cos 2 \beta y\right]+\check{P}^{\prime}=\Omega(\check{U}+\check{u}) .
\end{aligned}
$$

On taking the spanwise average of (6.9) we have

$$
W W^{\prime}+\check{P}^{\prime}=\Omega \check{U}
$$

and, hence,

$$
\begin{aligned}
& \check{u_{1}}=\frac{1}{\Omega \beta^{2}}\left(D^{2}-\beta^{2}\right)^{2} W(z), \\
& \check{u_{2}}=\frac{1}{4 \Omega \beta^{2}}\left(W^{\prime} W^{\prime \prime}-W W^{\prime \prime \prime}\right),
\end{aligned}
$$

where $D=\mathrm{d} / \mathrm{d} z$. For the present steady flow the mean flow equation (2.10) implies

$$
\frac{\mathrm{d} \breve{U}}{\mathrm{~d} z}=\overline{\check{w} \check{u}}-\frac{1}{2} \int_{-1}^{1} \overline{\text { w̌ }} \mathrm{d} z,
$$

in which, substituting for $\check{u}_{1}, \check{u}_{2}$ and $\check{w}$ from (6.11), (6.12) and (6.2), we have

$$
\overline{\check{w} \check{u}}=\frac{1}{2 \Omega \beta^{2}} W\left(D^{2}-\beta^{2}\right)^{2} W .
$$




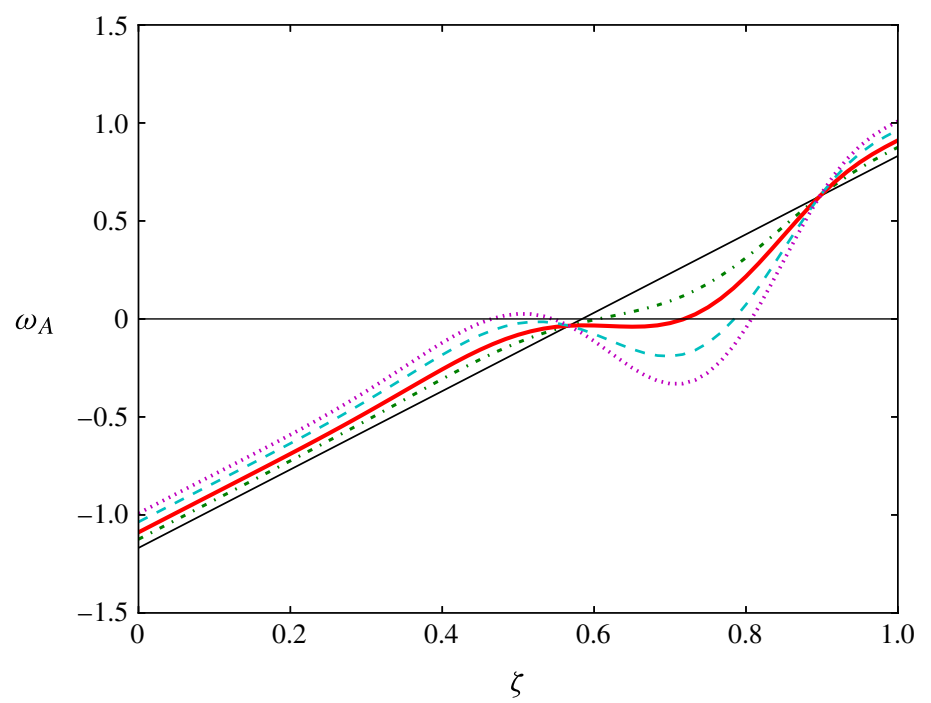

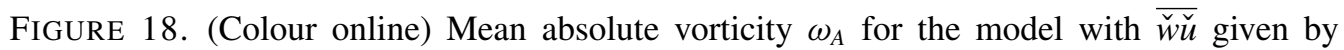
expression (6.14) as a function of $\zeta=(z+1) / 2$ for eigenfunction $W$ derived from the linear critical instability point $\left(R_{c}, \beta_{c}, \Omega_{c}\right)=(66.448,2.459,22.366)$. Amplitude scalings are:

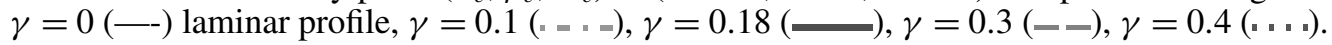

Substituting into expression (6.1) for $\mathrm{d} \check{U} / \mathrm{d} z$ from expression (6.13) with $\overline{\check{w} \check{u}}$ given by (6.14), we derive the model's approximation for $\omega_{A}$. Finally, the amplitude of the eigenfunction $W$ must also be specified. Introducing

$$
\gamma=\frac{|\check{U}(z=0)|}{\left|u_{0}(z=0)\right|},
$$

we plot in figure 18 profiles of $\omega_{A}$ obtained for this model solution for values of $\gamma$ in the range $0.1-0.4$. Clearly when $\gamma=0.18$ the model solution is returning an $\omega_{A}$ profile featuring a flattened central section similar to the full finite-amplitude results. Analysis of the full finite-amplitude results suggest this phenomenon is manifested when $\gamma>0.15$ or so, and so the amplitude scaling found for the model problem is of a consistent order of magnitude. We note also that we have also used $W$ derived instead from the full finite-amplitude solution, and obtained similar results.

The model can also capture the mechanism of the two-step $\omega_{A}$ profile shown in the plot for $\Omega=27.1$ in figure 15. The explanation lies in the fact that the lower branch of the solution shown in figure 15 bifurcates from the basic flow when the second least-stable eigenmode loses stability. With reference to figure 19, it can be seen that this second eigenfunction features a local minima as well as a local maxima in $0 \leqslant z \leqslant 1$, which produces the two-step profile for $\omega_{A}$ in contrast to the one-step profile produced by the least-stable eigenmode whose change in stability provides the bifurcation point for the upper branch of the nonlinear solutions. With reference to the velocity vectors for the full finite-amplitude solutions shown in figure 20, it can be seen that, moving along the upper branch from its bifurcation from the basic flow, initially only a single-layer roll-cell structure exists, which flattens $\omega_{A}$ in a region centred in $0 \leqslant z \leqslant 1$ with $\mathrm{d} U / \mathrm{d} z<0$. As the turning point of the nonlinear solution is approached these roll cells move towards the centre of the channel, and on traversing 


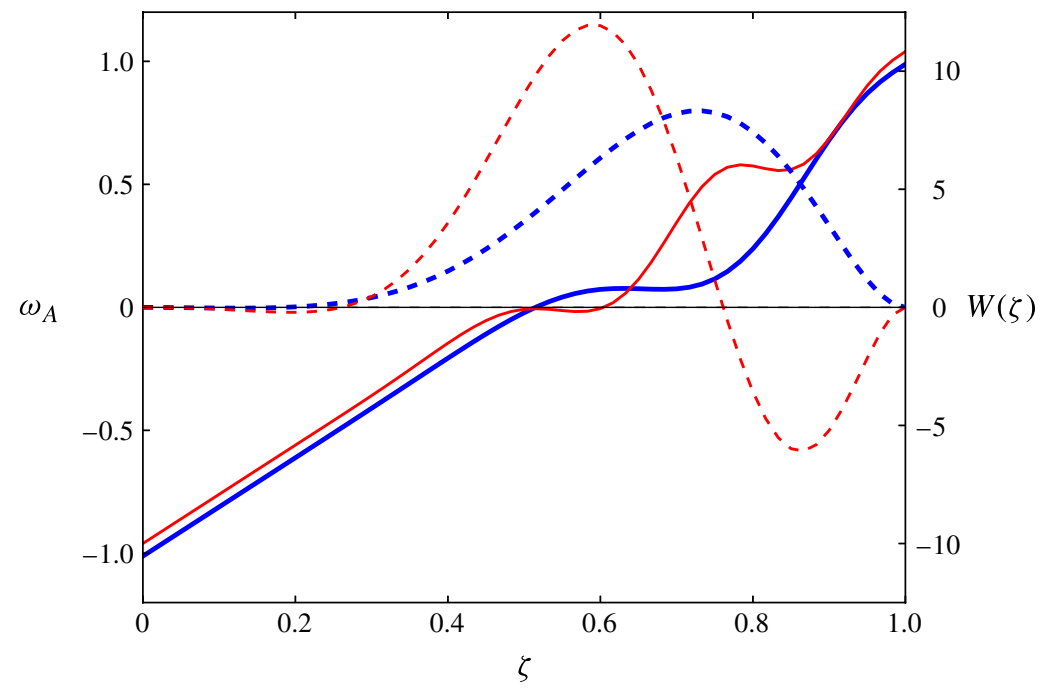

FIGURE 19. (Colour online) Two-dimensional model captures the one- and two-step flattening of the $\omega_{A}$ profile due to one- and two-layer streamwise vortex flows. Eigenfunction for $W$ from expression (6.5) with the sum truncated at $M=10$ (dashed lines, right-axis), and corresponding mean absolute vorticity $\omega_{A}$ (solid lines, left axis), as a function of $\zeta=(z+1) / 2$ calculated at bifurcation points of the secondary flow with $(\beta, \Omega)=(5.0,22.1325)$ from the basic flow at $R=110$ (single layer; thick lines, shown in blue online) and $R=589$ (double layer; thin lines, shown in red online).

the turning point, on the lower branch a second layer of roll cells then appears near the $z=1$ wall. With further movement towards the lower branch's bifurcation point from the basic flow the vortices adjacent to $z=1$ strengthen sufficiently to cause a second flattening of $\omega_{A}$, and thus the two-step profile described above.

The phenomenon given by the flattening of the profile of $\omega_{A}$ observed for many of the present transitional flows thus appears to differ from that observed for fully turbulent flows, in which the profile of $\omega_{A}$ is not just flattened, but, to the best of the present authors' knowledge, also approaches zero in all cases. It would thus seem that another mechanism operates in the fully turbulent regime. We note, however, that results showing a flattening of $\omega_{A}$ without approaching zero were also found by the DNS study of Iida et al. (2010) (see their figure 3(a) for example), who were considering the retransition of fully turbulent rotating channel flows. We also note the results of Oberlack (2001), who conducted a Lie-group analysis of the symmetries of the Reynolds-averaged Navier-Stokes equations for rotating turbulent channel flow, and found only that the mean flow became linear in a central region, i.e. that the profile of $\omega_{A}$ becomes flattened, with no requirement that $\omega_{A}$ approaches zero.

\section{Comparison with DNS and experimental studies}

An inspection of figures 4,8 and 11 reveals some of the difficulties of comparing the present finite-amplitude solutions with DNS and experimental results: even if we have accurate information concerning the physical parameters including wavenumbers, the existence of multiple bifurcation curves with the same parameter values, as well as multiple turning points leading to multiple solution branches along a single bifurcation curve, makes the selection of candidate solutions for comparison difficult. 
(a)

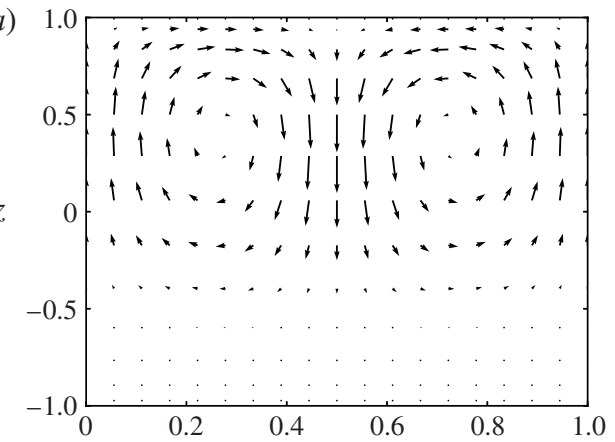

(c)

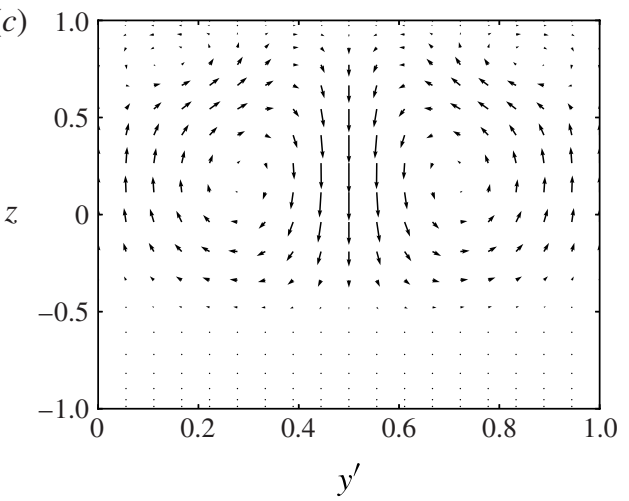

(b)

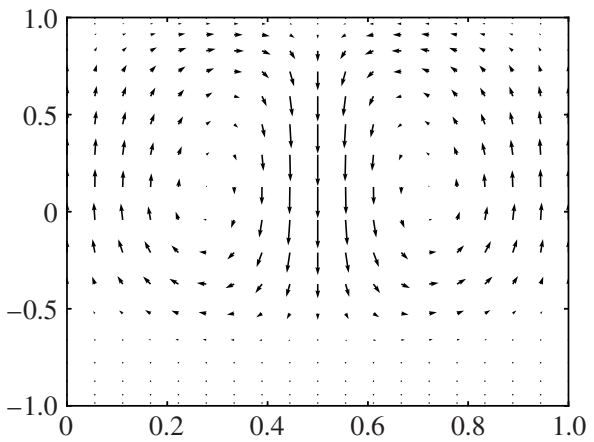

$(d)$

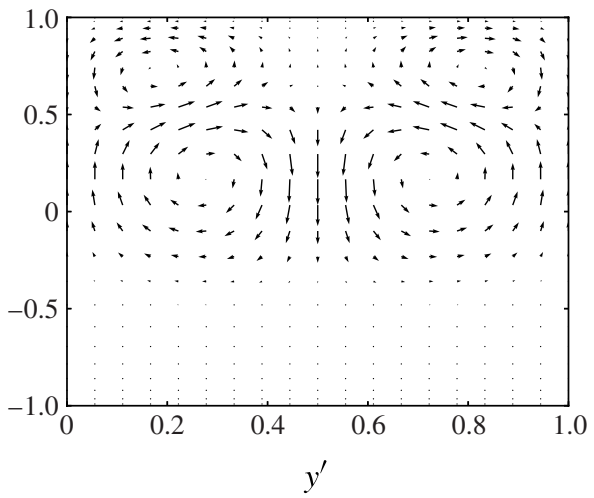

FIGURE 20. Changing structure of two-dimensional flows from a single layer of roll cells along the upper branch to a double layer of roll cells along the lower branch. Velocity vectors in spanwise-wall-normal plane for $(\beta, \Omega)=(5.0,22.1325)$ along the upper branch at $(a)$ $R=420,(b) R=2546$, lower branch solution at $(c) R=1511,(d) R=647$, computed at truncation level $(L, M, N)=(45,0,14)$. One wavelength is shown in $y^{\prime}=(1 / 2 \pi)(\beta y)$.

However, the DNS study of Finlay (1990), who chose parameter values in certain cases that also correspond to the experiments of Alfredsson \& Persson (1989), presents simulations that are performed close to the tertiary flow's bifurcation points, and so direct quantitative and qualitative comparisons are possible.

In his study, Finlay (1990) first analysed the stability of two-dimensional secondary flows generated by DNS to spanwise-superharmonic disturbances. Wall \& Nagata (2006) found good qualitative and quantitative agreement between these results and the stability of their flows found by the bifurcation approach. Finlay (1990) concluded his study by presenting simulations of the corresponding three-dimensional travelling-wave flows. We here compare the present tertiary flows obtained by the bifurcation method with these simulation results. Finlay (1990) classified their solutions as either 'WVF2' or 'WVF1', depending on whether the solution corresponded to the first or second unstable eigenvalue that became the most unstable eigenvalue with increasing $d$. Wall \& Nagata (2006) found good quantitative agreement with Finlay (1990) secondary stability results by considering the stability of upper-branch two-dimensional solutions, and so we seek superharmonic solutions bifurcating from such solutions for the present comparison, although for completeness solutions bifurcating from the lower branch were also considered. We note that it was stated that all of the simulated flows 
presented by Finlay (1990) satisfied symmetry (5.1), and so it is sufficient to consider only solutions of symmetry group $\mathscr{A}$ in the present comparison.

We first seek to compare with the flow presented in Finlay (1990, figure 9), which is a simulation with parameters chosen so as to compare with the experimental flow results presented by Alfredsson \& Persson (1989, figure 7(b)). In figure 21 velocity vectors in the spanwise-wall-normal plane at streamwise stations spanning half of one streamwise wavelength for an upper-branch superharmonic solution (flow type $\mathcal{G}_{1}$ ) are shown. It can be seen that, taking into account that the present channel rotation is in the opposite sense to that of Finlay (1990) study, the flow appears to be qualitatively the same as that shown in that study's figure 9. In particular, there are two vortices per spanwise wavelength, with the vortices moving away from the channel wall of the inviscidly unstable half of the channel as they become stronger, before becoming weaker and moving back towards this channel wall to complete one period of the flow pattern. The centres of these vortices show only a relatively small spanwise movement ('sideslip' according to Finlay (1990) terminology) that is less than 0.1 times the spanwise wavelength, and each vortex is strongest when the other vortex is weakest. For completeness, we have also computed the superharmonic flow with $d=O(1)$ bifurcating from the lower branch of the relevant secondary flow which has the same parameters as Finlay (1990, figure 9). This flow (not shown) is found to be qualitatively similar to that shown in figure $6(a)$, and so is qualitatively different to that shown in Finlay (1990, figure 9) and the present figure 21.

Finlay (1990) also presents quantitative data for the wavespeed, $c^{F I N}\left(=2 c / \operatorname{Re}^{F I N}\right)=$ 1.09 , for this flow, where $R e^{F I N}$ denotes Finlay's (1990) Reynolds number. For the flow shown in figure 21 and the corresponding lower-branch flow discussed above we obtain corresponding figures (expressed in Finlay's scales) of $c^{\text {FIN }}=1.09$ and $c^{F I N}=1.17$, respectively. For the same flow parameters Alfredsson \& Persson (1989) commented that the wavespeed of the experimentally observed flow was 'about half the undisturbed centreline velocity', or 0.75 expressed in Finlay's velocity scale. (Note that Alfredsson \& Persson (1989) did not state the wavenumbers for their flow, but these can be estimated from their figures.) Thus, overall it can be concluded that the upper-branch superharmonic $\mathcal{G}_{1}$ flow rather than the lower-branch superharmonic $\mathcal{G}_{2}$ flow offers the best agreement with the WVF1 simulation results presented in figure 9 of Finlay (1990) and figure 7(b) of Alfredsson \& Persson (1989), with good qualitative and quantitative agreement. (Note that the wavenumbers of the present solutions, $(\alpha, \beta)=(2.4,3.75)$, chosen from estimates taken from Alfredsson \& Persson (1989, figure $7(b))$, differ slightly from the values of Finlay $(1990),(\alpha, \beta)=(2,3)$.)

We also prepare a $\mathcal{G}_{3}$ flow in figure 21 with the same parameters as figure 10 of Finlay (1990) in order to compare with a 'WVF2' flow. The flow also appears to be qualitatively similar to the simulation results: there is a larger sideways oscillation (greater than 0.3 times the spanwise wavelength) than vertical oscillation of the centres of the vortices, with each of the two vortices strongest approximately at the time the other is weakest and with the vortices almost disappearing at the weakest point of their cycle. For this flow Finlay (1990) obtains $c^{F I N}=1.25$, while our flow has $c^{F I N}=1.24$. We thus again conclude good qualitative and quantitative agreement between the present results and the WVF2 results of Finlay (1990).

We also consider a comparison with the simulation study of Yang \& Kim (1991). Yang \& Kim (1991) follow the evolution in time of an initial flow of laminar Poiseuille flow with a small-amplitude disturbance added that is subject to a spanwise rotation that is suddenly switched on at $t=0$. Yang \& Kim (1991) considered four main parameter cases, with the majority of results presented for 

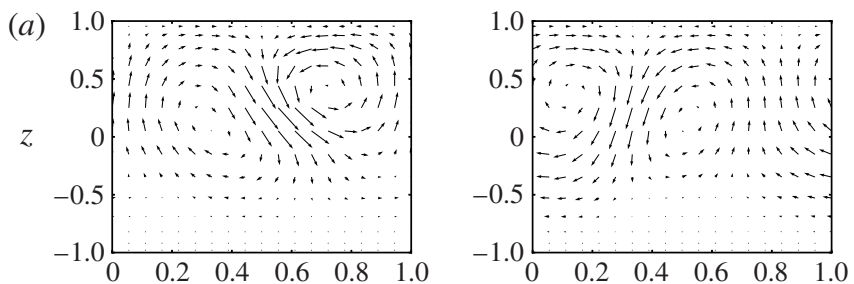

(b)
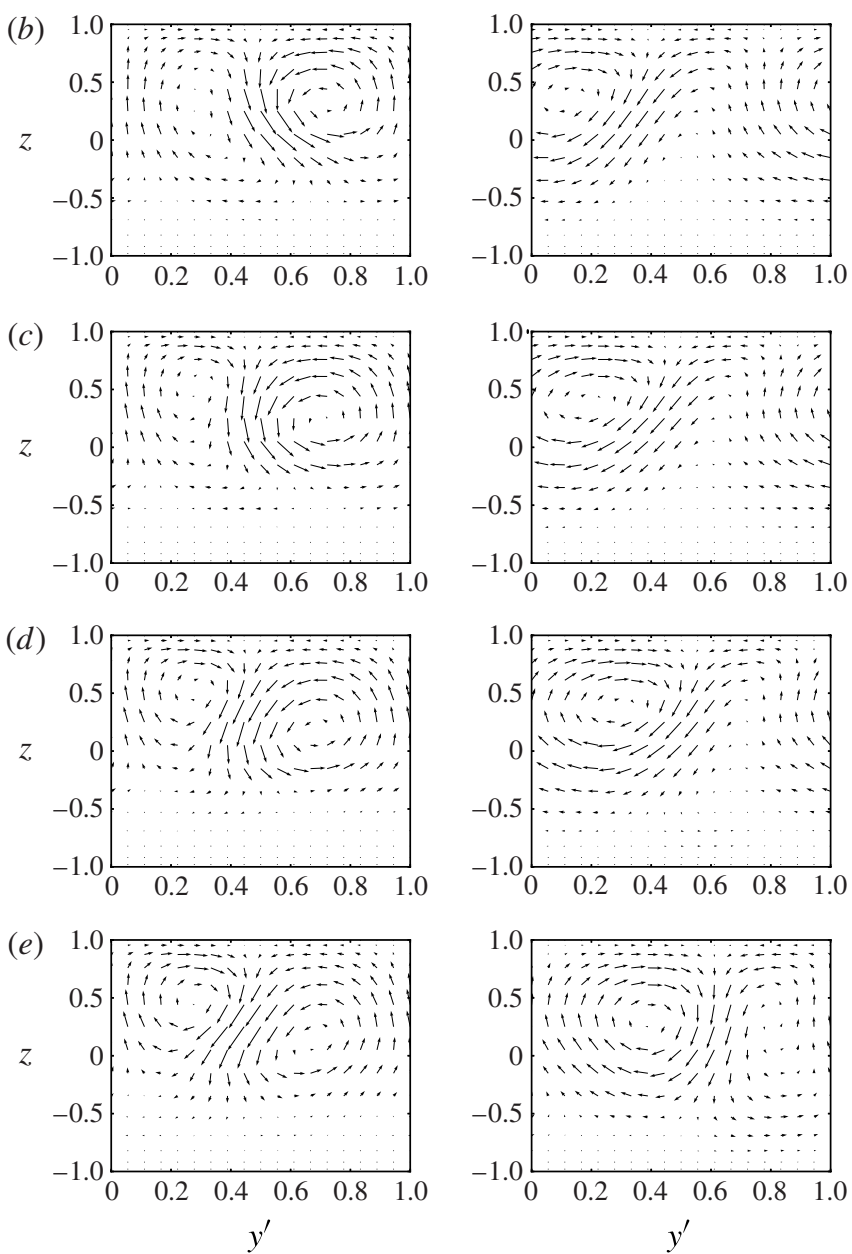

FIGURE 21. Velocity vector plots in the spanwise-wall-normal plane at streamwise stations $x=(2 \pi / \alpha)(i / 10),(a) i=0,(b) i=1$, (c) $i=2,(d) i=3$, (e) $i=4$ for the (left) upper-branch superharmonic tertiary flow, flow type $\mathcal{G}_{1}$, with parameters $(R, \alpha, \beta, \Omega)=$ (683.6, 2.4, 3.75, 24.75), chosen to compare with the flow presented by Finlay (1990, figure $9)$, and (right) flow type $\mathcal{G}_{3},(R, \alpha, \beta, \Omega)=(259,0.4,3.0,14.0)$, chosen to compare with the flow presented in Finlay (1990, figure 10). Both flows computed at $(L, M, N)=(29,8,8)$. One wavelength is shown in $y^{\prime}=(1 / 2 \pi)(\beta y)$. 
case I $\left((\alpha, \beta, \Omega)=(2.17,1.5,23.65)\right.$, and with $R$ chosen so that $\int_{-1}^{1} u \mathrm{~d} z=2 * R e^{Y K}=$ 946), which appears to be a stable three-dimensional 'twisting' flow. In figure $9(\mathrm{~d})$ of Yang \& Kim (1991) it can be seen that this flow features staggered vortices in the streamwise direction, while an examination of the velocity vector plots presented in their figure 9 reveals that their flow has two vortices per spanwise wavelength and satisfies the glide-reflectional symmetry satisfied by superharmonic solutions of group $\mathscr{A}$. Yang \& Kim (1991) also found that their case I flow was characterized by a small sideways movement of the centres of the vortices, with each vortex becoming alternately strongest when the line connecting the centres of a vortex pair lies parallel to the spanwise axis. We can further observe from Yang \& Kim (1991) plots of the fluctuating components of velocity in their figure 8 that the streamwise component is distinguished by one low-speed streak of curved profile per spanwise wavelength, with no significant areas of high-speed flow between these streaks. Only the $\mathcal{G}_{1}$ superharmonic flow appears to match all of these qualitative features. A quantitative comparison is, unfortunately, difficult: the most obvious approach is to seek a three-dimensional flow that bifurcates from a two-dimensional streamwiseindependent $\hat{\beta}=1.5$ flow in a spanwise-superharmonic bifurcation with a streamwise secondary disturbance wavenumber parameter $d=2.17$. However, the $\grave{\beta}=1.5$ twodimensional flow that bifurcates from the basic state (at $R=76.7$ ) terminates at $R=95.3$, and is everywhere linearly stable to a spanwise-superharmonic disturbance with $d=2.17$.

However, we note that the results of this section would thus suggest that the present superharmonic flows bifurcating from the upper branch of the secondary flow, for both $d=O(1)$ (flow type $\mathcal{G}_{1}$ ) and $d=O\left(10^{-1}\right.$ ) (flow type $\mathcal{G}_{3}$ ), appear to be qualitatively similar to the simplest tertiary flows observed in DNS and experimental studies.

\section{Conclusions}

For the problem of channel flow subject to a system rotation about a spanwise axis, this study has presented exact, finite-amplitude solutions for three-dimensional travelling wave flows. Both tertiary flows, which bifurcate from steady twodimensional streamwise-independent secondary flows, and secondary flows, which bifurcate directly from the basic state, are presented.

For the tertiary flows, for fixed $\Omega$, bifurcations from the secondary flow with spanwise wavenumber $\grave{\beta}=2.5$ were considered, with the results representative of tertiary flows bifurcating from secondary flows with $2 \leqslant \grave{\beta} \leqslant 4$, an interval which in particular includes all of the stable secondary flows. Solutions of superharmonic (i.e. the spanwise secondary disturbance wavenumber parameter $b=\hat{\beta}$ ) and subharmonic (i.e. $b=\grave{\beta} / 2$ ) type are found. Regarding the streamwise secondary disturbance wavenumber parameter $d$, it was found convenient and sufficient to examine two regimes, the $d=O\left(10^{-1}\right)$ regime, the interval of smaller values of $d$ in which the maximum number of concurrently unstable eigenmodes is possible, and the $d=O(1)$ regime, in which a single eigenmode destabilizes on the upper branch of the secondary flow and restabilizes on the lower branch. Two symmetry groups are possible for the superharmonic flows: group $\mathscr{A}$ (see appendix A for precise definitions of the symmetry groups) solutions satisfy a glide-reflectional symmetry in the $y=\pi / \beta$ plane, where $y$ denotes the spanwise distance in a Cartesian system, while group $\mathscr{B}$ solutions satisfy a reflectional symmetry about the same plane. For the subharmonic solutions, and also for the three-dimensional secondary flows, only one symmetry group, $\mathscr{C}$, 
is possible, and these solutions satisfy a glide-reflectional symmetry about the plane $y=\pi /(2 \beta)$, and also a reflectional symmetry about the plane $y=\pi / \beta$.

However, the above symmetry rules are not sufficient to distinguish between distinct flows within the same symmetry class. Every tertiary (secondary) flow presented here arises in a bifurcation when a secondary (linear) eigenmode changes stability. It is found that different eigenmodes produce different flows, while different flows are also observed on different major branches of the same bifurcation curve (where a bifurcation curve has two major branches if it connects (i.e. bifurcates from) two different flows and features at least one turning point). Distinguishing the flows according to these three criteria, symmetry group, originating eigenmode and solution branch, this study has identified a total of 15 distinct three-dimensional flows for this problem: there are five distinct superharmonic tertiary flows, five distinct subharmonic tertiary flows and five secondary flows. The main structural features of these flows are summarized in table 3 .

The main findings of this study are as follows.

(i) The tertiary flows all feature a single layer of vortical structures in the spanwise-wall-normal plane, which is generally located in the inviscidly unstable (i.e. anticyclonic) half of the channel. The three-dimensional secondary flows feature single, double, triple or quadruple layers of vortical structures, with the number of layers increasing the higher the order of the eigenmode whose change in stability provides the bifurcation point.

(ii) The structures of all of the flows feature low-speed streamwise-orientated streaks in the streamwise velocity component and/or pulses of low-speed streamwise velocity. The streaks may be either sinusoidal or varicose. High-speed streaks and pulses can also appear, and have been found to dominate in some layers of the secondary flows when stronger than the low-speed streaks/pulses.

(iii) Sinusoidal streaks are always flanked by staggered vortices, while varicose streaks, as well as pulses, are always flanked by aligned vortices. The staggered vortices undergo a cycle of strengthening and weakening in which the vortex on one side of the streak is strongest when the vortex on the other side is weakest. The aligned vortices are, by contrast, mirror symmetric about the streak's centreline.

(iv) Adjacent streaks can merge if their spanwise variation is sufficiently large, resulting in more complicated flow patterns.

(v) It is found that for many of the present flows mean absolute vorticity, $\omega_{A}$, adopts a flat (i.e. constant) profile across a central region of the channel that is centred in the anticyclonic half of the channel. However, in contrast to fully turbulent flow, it is not the case that $\omega_{A}$ is vanishingly small in such regions, and indeed flows exist for which two-step $\omega_{A}$ profiles are observed in which $\omega_{A}$ is $O(1)$ in one of the steps. Further investigation reveals that this phenomenon is more clearly manifested in two-dimensional secondary flows bifurcating from the basic state than the tertiary flows, while, of the latter, flows featuring unbroken low-speed streaks of smaller spanwise variance more clearly manifest the phenomenon. We proposed that the phenomenon can be explained by a two-dimensional mechanism given by mixing across the vortex layer(s), and presented a two-dimensional model in which a simple prescribed vortex flow system yields a flattened $\omega_{A}$ profile similar to the full finite-amplitude results.

(vi) A comparison with previous DNS studies was conducted, and it is suggested that two of the simpler flows that have been observed in the simulations are given by 


\begin{tabular}{|c|c|c|c|c|c|c|c|c|}
\hline $\begin{array}{l}\text { Flow } \\
\text { type }\end{array}$ & SYM & Type & $O(d)$ & Layers & Branch & Streaks & $\begin{array}{l}\text { Spanwise } \\
\text { fluctuation }\end{array}$ & Pulses \\
\hline $\mathcal{G}_{1}$ & $\mathscr{A}$ & SUP & $O(1)$ & 1 & Upper & $\mathrm{S}, 1$ & Small & - \\
\hline $\mathcal{G}_{2}$ & $\mathscr{A}$ & SUP & $O(1)$ & 1 & Lower & $\mathrm{S}, 2$ & Small & - \\
\hline $\mathcal{G}_{3}$ & $\mathscr{A}$ & SUP & $O\left(10^{-1}\right)$ & 1 & Upper & $\mathrm{S}, 1$ & Large & - \\
\hline $\mathcal{G}_{4}$ & $\mathscr{A}$ & SUP & $O\left(10^{-1}\right)$ & 1 & Lower & $\mathrm{S}, 1$ & Small & $\mathrm{P}, 2 \times 1$ \\
\hline $\mathcal{G}_{5}$ & $\mathscr{B}$ & SUP & $O\left(10^{-1}\right)$ & 1 & Lower & $\mathrm{V}, 1$ & - & $\mathrm{P}, 1 \times 1$ \\
\hline $\mathcal{G}_{6}$ & $\mathscr{C}$ & SUB & $O(1)$ & 1 & Upper & $\mathrm{S}, 2$ & Small & - \\
\hline $\mathcal{G}_{7}$ & $\mathscr{C}$ & SUB & $O(1)$ & 1 & Lower & $\mathrm{S}, 2+\mathrm{V}, 2$ & Small & - \\
\hline $\mathcal{G}_{8}$ & $\mathscr{C}$ & SUB & $O\left(10^{-1}\right)$ & 1 & Upper & $\mathrm{S}, 2$ & Large & $\mathrm{P}, 1 \times 2$ \\
\hline $\mathcal{G}_{9}$ & $\mathscr{C}$ & SUB & $O\left(10^{-1}\right)$ & 1 & Upper & $\mathrm{S}, 2$ & Large & - \\
\hline $\mathcal{G}_{10}$ & $\mathscr{C}$ & SUB & $O\left(10^{-1}\right)$ & 1 & Lower & $\mathrm{S}, 2$ & Large & $\mathrm{P}, 1 \times 2$ \\
\hline $\mathcal{G}_{11}$ & $\mathscr{C}$ & SEC & - & 1 & - & - & - & $\mathrm{P}, 1 \times 2$ \\
\hline $\mathcal{G}_{12}$ & $\mathscr{C}$ & SEC & - & 1 & - & $\mathrm{V}, 2$ & - & - \\
\hline $\mathcal{G}_{13}$ & $\mathscr{C}$ & SEC & - & 2 & - & - & - & $\begin{array}{l}\mathrm{P}, 1 \times 2^{a} \\
\mathrm{P}, 1 \times 2\end{array}$ \\
\hline $\mathcal{G}_{14}$ & $\mathscr{C}$ & SEC & - & 2 & - & $\begin{array}{l}\mathrm{T}, 2 \\
\mathrm{~V}, 2\end{array}$ & - & - \\
\hline $\mathcal{G}_{15}$ & $\mathscr{C}$ & SEC & - & 4 & - & $\begin{array}{l}\mathrm{V}, 2 \\
- \\
-\end{array}$ & $\begin{array}{l}- \\
-\end{array}$ & $\begin{array}{c}- \\
\mathrm{P}, 1 \times 2 \\
\mathrm{P}, 1 \times 2^{a} \\
\mathrm{P}, 1 \times 2\end{array}$ \\
\hline
\end{tabular}

TABLE 3. Summary of the main features of the 15 distinct three-dimensional flows. Here 'type' may be tertiary superharmonic (SUP), tertiary subharmonic (SUB) or a secondary flow (SEC), while 'SYM' refers to the symmetry groups listed in appendix A. The 'branch' entry lists the branch of the two-dimensional secondary flow the tertiary flows bifurcate from, 'layers' the number of layers the flow structure has in the spanwise-wallnormal plane, the 'streaks' entry details the type of low-speed streak, either sinusoidal $(\mathrm{S})$, varicose $(\mathrm{V})$ or quasi-two-dimensional $(\mathrm{T})$, and the number of streaks per spanwise wavelength. The spanwise fluctuation of sinusoidal streaks is labelled 'large' if it extends across a whole spanwise wavelength (or half-wavelength in the case of S,2), and 'small' otherwise. In the final column ' $\mathrm{P}, a \times b$ ' denotes $b$ lines of pulses per spanwise wavelength with each line featuring $a$ pulses per streamwise wavelength. For multiple-layered flows the structure of each layer is listed in order from the $z=1$ wall. ${ }^{a}$ In these cases the pulses are high-speed, in all other cases they are low-speed.

the superharmonic flows bifurcating from the upper branch when $d=O(1)\left(\mathcal{G}_{1}\right.$ in the present notation), and $d=O\left(10^{-1}\right)\left(\mathcal{G}_{3}\right.$ in the present notation).

One direction for future work is to conduct appropriate stability analysis to confirm the final conclusion above. Many of the other flows are likely to be unstable. However, such flows, even if not stable, may nonetheless play an important role in explaining phenomena observed in experimental and DNS studies, which report more complicated flow patterns as $R$ is increased or with changes in the value of $\Omega$. For example, Yang \& Kim (1991) describe a simulation (their case III) in which a three-dimensional flow featuring both $d=O(1)$ and $d=O\left(10^{-1}\right)$ flow structures is observed, for which an explanation formulated in terms of a heteroclinic orbit between two or more of the present solutions may be possible. 
Other directions for future work include the exploration of whether any of the present flows may be continued by homotopy to other flow configurations, including plane Couette flow with spanwise rotation, or plane Poiseuille flow in the absence of rotation.

\section{Acknowledgements}

We would like to thank the referees for the improvements to the manuscript that resulted from responding to their comments.

\section{Supplementary movies}

Supplementary movies are available at http://dx.doi.org/10.1017/jfm.2013.242.

\section{Appendix A. Symmetry groups}

The following notation has been adopted: a double dash $\left({ }^{\prime \prime}\right)$ denotes an even integer, a single dash $\left(^{\prime}\right)$ denotes an odd integer, while $e(z)$ denotes an even single-variable function of $z$ and $o(z)$ denotes an odd single-variable function of $z$.

Superharmonic tertiary flows

Group $\mathscr{A}$ :

$$
\begin{aligned}
& \left(\begin{array}{c}
\cos m^{\prime \prime} \alpha(x-c t) \\
\sin m^{\prime \prime} \alpha(x-c t)
\end{array}\right)\left(\begin{array}{c}
\cos n^{\prime} \beta y \\
\cos n^{\prime \prime} \beta y
\end{array}\right)\left(\begin{array}{l}
o(z) \\
e(z)
\end{array}\right) \mid\left(\begin{array}{l}
\cos m^{\prime \prime} \alpha(x-c t) \\
\sin m^{\prime \prime} \alpha(x-c t)
\end{array}\right)\left(\begin{array}{c}
\sin n^{\prime} \beta y \\
\sin n^{\prime \prime} \beta y
\end{array}\right)\left(\begin{array}{l}
o(z) \\
e(z)
\end{array}\right) \\
& \left(\begin{array}{c}
\cos m^{\prime} \alpha(x-c t) \\
\sin m^{\prime} \alpha(x-c t)
\end{array}\right)\left(\begin{array}{c}
\sin n^{\prime} \beta y \\
\sin n^{\prime \prime} \beta y
\end{array}\right)\left(\begin{array}{l}
o(z) \\
e(z)
\end{array}\right) \mid\left(\begin{array}{c}
\cos m^{\prime} \alpha(x-c t) \\
\sin m^{\prime} \alpha(x-c t)
\end{array}\right)\left(\begin{array}{c}
\cos n^{\prime} \beta y \\
\cos n^{\prime \prime} \beta y
\end{array}\right)\left(\begin{array}{l}
o(z) \\
e(z)
\end{array}\right) .
\end{aligned}
$$

Group $\mathscr{B}$ :

$$
\begin{gathered}
\phi \\
\left(\begin{array}{c}
\cos m^{\prime \prime} \alpha(x-c t) \\
\cos m^{\prime} \alpha(x-c t) \\
\sin m^{\prime} \alpha(x-c t) \\
\sin m^{\prime \prime} \alpha(x-c t)
\end{array}\right)\left(\begin{array}{c}
\cos n^{\prime} \beta y \\
\cos n^{\prime \prime} \beta y
\end{array}\right)\left(\begin{array}{c}
o(z) \\
e(z)
\end{array}\right) \mid\left(\begin{array}{c}
\cos m^{\prime \prime} \alpha(x-c t) \\
\cos m^{\prime} \alpha(x-c t) \\
\sin m^{\prime} \alpha(x-c t) \\
\sin m^{\prime \prime} \alpha(x-c t)
\end{array}\right)
\end{gathered}
$$

Subharmonic tertiary flow

(Includes three-dimensional secondary flows bifurcating directly from the basic state.) Group $\mathscr{C}$ :

$$
\begin{aligned}
& \left(\begin{array}{c}
\cos m^{\prime \prime} \alpha(x-c t) \\
\sin m^{\prime \prime} \alpha(x-c t)
\end{array}\right)\left(\begin{array}{l}
\phi \\
\left(\cos n^{\prime \prime} \beta y\right)
\end{array}\right)\left(\begin{array}{c}
o(z) \\
e(z)
\end{array}\right) \mid\left(\begin{array}{c}
\cos m^{\prime \prime} \alpha(x-c t) \\
\sin m^{\prime \prime} \alpha(x-c t)
\end{array}\right)\left(\begin{array}{l}
\sin n^{\prime \prime} \beta y
\end{array}\right)\left(\begin{array}{l}
o(z) \\
e(z)
\end{array}\right) \\
& \left(\begin{array}{c}
\cos m^{\prime} \alpha(x-c t) \\
\sin m^{\prime} \alpha(x-c t)
\end{array}\right) \quad\left(\begin{array}{l}
\cos n^{\prime} \beta y
\end{array}\right)\left(\begin{array}{c}
o(z) \\
e(z)
\end{array}\right)\left(\begin{array}{c}
\cos m^{\prime} \alpha(x-c t) \\
\sin m^{\prime} \alpha(x-c t)
\end{array}\right)\left(\begin{array}{l}
\sin n^{\prime} \beta y
\end{array}\right)\left(\begin{array}{l}
o(z) \\
e(z)
\end{array}\right) .
\end{aligned}
$$




\section{Appendix B. Analytical solution to linear stability problem}

For the model problem described in $\S 6$ it is necessary to derive an analytical expression for the eigenfunctions of the linear stability problem for the case of twodimensional streamwise-independent disturbances. Seeking a solution for the normalmode linear disturbance to $w$ in the form $\check{w}=W(z) \mathrm{e}^{\mathrm{i}(\alpha x+\beta y)+\sigma t}$ we have from (10) and (16) of Wall \& Nagata (2006) that $W=\beta^{2} \Phi$, and it is then straightforward to derive from the linear stability problem expressed by (18) and (19) subject to boundary conditions (20) of Wall \& Nagata (2006) with $\alpha=0$ that

$$
\left(D^{2}-\beta^{2}-\sigma\right)^{2}\left(D^{2}-\beta^{2}\right) W-\beta^{2} \Omega^{2}\left(1-\frac{2 R z}{\Omega}\right) W=0,
$$

where $D=\mathrm{d} / \mathrm{d} z$. For the present purposes we are interested in steady, marginal solutions $(\sigma=0)$, and upon introducing the substitutions

$$
T=-16 \Omega(\Omega+2 R), \quad a=2 \beta, \quad \chi=\frac{-4 R}{\Omega+2 R}, \quad \zeta=\frac{z+1}{2},
$$

equation (B 1) becomes

$$
\left(E^{2}-a^{2}\right)^{3} W+a^{2} T(1+\chi \zeta) W=0,
$$

in which $E=\mathrm{d} / \mathrm{d} \zeta$, while the non-slip boundary conditions become

$$
W=W^{\prime}(\zeta)=E^{2}\left(E^{2}-2 a^{2}\right) W=0 \quad \text { at } \zeta=0,1 .
$$

Following the approach of Chandrasekhar (1981), we re-express problem (B 3) as

$$
\begin{gathered}
S=-a^{2} T W, \\
\left(E^{2}-a^{2}\right)^{3} W=(1+\chi \zeta) S .
\end{gathered}
$$

Seeking solutions for $S$ in the form

$$
S=\sum_{m=1}^{\infty} c_{m} \sin m \pi \zeta
$$

and for $W$ in the form of expression (6.5), we are left to solve

$$
\left(E^{2}-a^{2}\right)^{3} w_{m}=(1+\chi \zeta) \sin m \pi \zeta
$$

for which the general solution is given by expression (6.6). For the present boundary conditions, after a little algebra, we find

$$
\begin{aligned}
A_{1}^{(m)}= & -\frac{6 \chi m \pi}{m^{2} \pi^{2}+a^{2}} \\
A_{3}^{(m)}= & -\frac{\chi m \pi}{4 a^{2}}\left(m^{2} \pi^{2}+a^{2}\right) \\
B_{3}^{(m)}= & \frac{\chi m \pi\left(m^{2} \pi^{2}+a^{2}\right)}{4 a^{2} \sinh a}\left(\cosh a-(-1)^{m}\right) \\
B_{1}^{(m)}= & \frac{m \pi}{4 a^{2}\left(m^{2} \pi^{2}+a^{2}\right)\left(a^{2}-\sinh ^{2} a\right)}\left\{\pi ^ { 2 } m ^ { 2 } \left[\pi^{2} m^{2} \chi\left((-1)^{m} \sinh a-a\right)\right.\right. \\
& \left.-2 a^{2}(\chi+2)\left((-1)^{m} \sinh a+a\right)\right]+a^{2}\left[1 2 \chi \left(2(\sinh a+a \cosh a)(-1)^{m}\right.\right. \\
& \left.\left.-\sinh 2 a-2 a)-a^{2}(-1)^{m} \sinh a(3 \chi+4)-a^{3}(\chi+4)\right]\right\}
\end{aligned}
$$




$$
\begin{aligned}
A_{2}^{(m)}= & -m \pi-a B_{1} \\
B_{2}^{(m)}= & \frac{m \pi}{4 a^{2}\left(m^{2} \pi^{2}+a^{2}\right)\left(\sinh ^{2} a-a^{2}\right) \sinh a}\left\{\pi^{4} m^{4} \chi\right. \\
& \left(\left[\sinh a(2 \sinh a-a \cosh a)-a^{2}\right](-1)^{m}+a(a \cosh a-\sinh a)\right) \\
& +\pi^{2} m^{2} a^{2}\left((a \cosh a-\sinh a)\left(2 a \chi+(-1)^{m} 4 \sinh a\right)\right. \\
& \left.+(\sinh 2 a-2 a)\left(a \chi(-1)^{m}+2 \sinh a\right)\right) \\
& +a^{3}\left(\left[(-1)^{m}\left(-26 a \sinh ^{2} a+3 a^{2} \cosh a \sinh a-a^{3}\right)\right.\right. \\
& \left.+\left(24 \sinh ^{3} a-a^{2} \sinh a+a^{3} \cosh a\right)\right] \chi \\
& \left.\left.+2 a \sinh a\left[\sinh 2 a-2 a+(-1)^{m} 2(a \cosh a-\sinh a)\right]\right)\right\} .
\end{aligned}
$$

In order obtain the $c_{m}$ coefficients we substitute into (B 5) for $S$ from expression (B 7) to obtain

$$
\sum_{m=1}^{\infty} c_{m} \sin m \pi \zeta=-a^{2} T \sum_{m=1}^{\infty} c_{m} w_{m} .
$$

Finally, applying the operator $\int_{0}^{1} \sin p \pi \zeta \mathrm{d} \zeta$ to both sides of (B 15) we obtain the eigenvalue problem

$$
\mathscr{A}_{m, n} c_{n}=\lambda c_{m}
$$

for eigenvalues $\lambda=1 / T$ where

$$
\mathscr{A}_{m, n}=-2 a^{2} \int_{0}^{1} w_{n} \sin m \pi \zeta \mathrm{d} \zeta .
$$

Only a few terms of the series (6.5), (B 7) are necessary to achieve a small relative error in the eigenmodes. For example, just three terms are sufficient to return a relative error of $O\left(10^{-2}\right)$ for the linear critical point determined by Wall \& Nagata (2006) to be $\left(R_{c}, \beta_{c}, \Omega_{c}\right)=(66.448,2.459,22.366)$.

\section{REFERENCES}

Alfredsson, P. H. \& Persson, H. 1989 Instabilities in channel flow with system rotation. J. Fluid Mech. 202, 543-557.

Chandrasekhar, S. 1981 Hydrodynamic and Hydromagnetic Stability. Dover.

DiPRIMA, R. C. \& SWINNEY, H. L. 1985 Instabilities and transition in flow between concentric rotating cylinders. In Hydrodynamic Instabilities and the Transition to Turbulence (ed. H. L. Swinney \& J. P. Gollub), Topics in Applied Physics, vol. 45. pp. 139-180. Springer.

FAisst, H. \& ECKhardt, B. 2003 Traveling waves in pipe flow. Phys. Rev. Lett. 91, 224502.

FINLAY, W. H. 1990 Transition to oscillatory motion in rotating channel flow. J. Fluid Mech. 215, 209-227.

FInLAY, W. H. 1992 Transition to turbulence in a rotating channel. J. Fluid Mech. 237, 73-99.

Finlay, W. H., Keller, J. B. \& Ferziger, J. H. 1988 Instability and transition in curved channel flow. J. Fluid Mech. 194, 417-456.

Gibson, J. F., Halcrow, J. \& CVitanovic, P. 2009 Equilibrium and travelling-wave solutions of plane Couette flow. J. Fluid Mech. 638, 243-266.

Hopfinger, E. J. \& LindEN, P. F. 1990 The effect of background rotation on fluid motions: a report on Euromech 245. J. Fluid Mech. 211, 417-435.

Iida, O., Fukudome, K., Iwata, T. \& Nagano, Y. 2010 Low Reynolds number effects on rotating turbulent Poiseuille flow. Phys. Fluids 22, 085106.

KERSWELL, R. R. 2005 Recent progress in understanding the transition to turbulence in a pipe. Nonlinearity 18, R17-R44. 
Kristoffersen, R. \& ANDERSSON, H. I. 1993 Direct simulations of low-reynolds-number turbulent flow in a rotating channel. J. Fluid Mech. 256, 163-197.

Lesieur, M., Yaglom, A. M. \& David, F. (Eds) 2002 New Trends in Turbulence. Springer.

Lezius, D. K. \& Johnstone, J. P. 1976 The structure and stability of turbulent boundary layers in rotating channel flow. J. Fluid Mech. 77, 153-175.

Matsubara, M. \& Alfredsson, P. H. 1998 Secondary instability in rotating channel flow. J. Fluid Mech. 368, 27-50.

NagATA, M. 1990 Three-dimensional finite amplitude solutions in plane Couette flow. J. Fluid Mech. 217, 519-527.

NagATA, M. 1998 Tertiary solutions and their stability in rotating plane Couette flow. J. Fluid Mech. 358, 357-378.

OBERLACK, M. 2001 A unified approach for symmetries in plane parallel turbulent shear flows. J. Fluid Mech. 427, 299-328.

Pedley, T. J. 1969 On the instability of viscous flow in a rapidly rotating pipe. J. Fluid Mech. 35, 97-115.

Tsukahara, T., Tillmark, N. \& Alfredsson, P. H. 2010 Flow regimes in a plane Couette flow with system rotation. J. Fluid Mech. 648, 5-33.

WALefFe, F. 2001 Exact coherent structures in channel flow. J. Fluid Mech. 435, 93-102.

WALl, D. P. \& NAGATA, M. 2006 Nonlinear secondary flow through a rotating channel. J. Fluid Mech. 564, 25-55.

Wedin, H. \& KERSWELL, R. R. 2004 Exact coherent structures in pipe flow: travelling wave solutions. J. Fluid Mech. 508, 333-371.

YANASE, S. \& KAGA, Y. 2004 Zero-mean-absolute-vorticity state and vortical structures in rotating channel flow. J. Phys. Soc. Jpn 73, 1419-1422.

YANG, K. S. \& KIM, J. 1991 Numerical investigation of instability and transition in rotating plane Poiseuille flow. Phys. Fluids A 3, 633-641. 